\title{
Evaluation of the Efficacy of Head and Brain Injury Risk Functions
}

\author{
A Thesis \\ Presented to
}

the faculty of the School of Engineering and Applied Science

University of Virginia

\author{
in partial fulfillment \\ of the requirements for the degree \\ Master of Science \\ by
}

Erin Jo Sanchez

December 2017 


\title{
APPROVAL SHEET
}

\author{
This Thesis \\ is submitted in partial fulfillment of the requirements \\ for the degree of \\ Master of Science
}

Author Signature: Eim fanehey

This Thesis has been read and approved by the examining committee:

Advisor: Jeff Crandall

Committee Member: Matt Panzer

Committee Member: Tim McMurry

Committee Member:

Committee Member:

Committee Member:

Accepted for the School of Engineering and Applied Science:

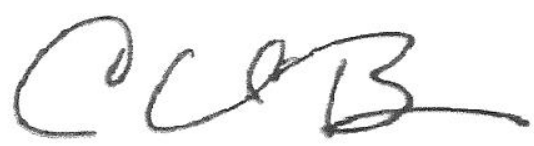

Craig H. Benson, School of Engineering and Applied Science

December 2017 


\begin{abstract}
Head and brain injury risk functions have been proposed over the years in order to estimate the probability of brain injury from head impact using kinematic and injury data from various sources, such as PMHS and animal testing. Yet, researchers have reiterated the need for human head kinematic data for creating and evaluating injury risk functions and risk assessment values given that other types of data may lack the physiologic and anthropometric response required.
\end{abstract}

To this end, this thesis aimed to collect human head kinematics to evaluate existing injury risk functions. A literature review revealed what measures of human head motion have been previously published, including direct measures of head kinematics (e.g., wearable sensors used on-field with contact athletes) and indirect measures of head motion (e.g., computational and experimental reconstructions of real-life impacts). Three data sources $(n=443)$ were selected from the literature based on inclusion criteria. Data were evaluated for consistency and used to calculate kinematic and strain-based injury risks.

The injury risk values were used to evaluate the efficacy of each of 16 injury risk functions using four separate analytical tools. Correlations between the injury risk functions and strain metrics showed that strain- and rotationally-based injury risk functions had the strongest correlations. Area under ROC curves assessed each function's ability to separate injurious and non-injurious impacts; all risk functions were better than random guessing. Likelihood estimates ranked the injury risk functions on their ability to correctly predict the dataset's injury outcomes, with GAMBIT showing the best predictive capability according to this measure. The number of expected injuries was calculated for each risk function; however, most did not correctly estimate the number of observed injuries $(n=31)$. Results from these four assessment tools, however, were mixed, with no single risk function performing best.

The lack of consensus in the assessment tools may be a result of the data used to develop the risk functions. Studies have noted unbiased exposure data is needed to estimate the absolute injury risk from impact; however, most injury risk functions (and the data in this thesis) were based on case-control data. Statistical simulations were conducted to create injury risk functions based on several sampling scenarios. These results from these simulations demonstrated that researchers should be wary of risk curves derived solely from case-controlled data given that these may be over-predictive of injury probabilities compared to the absolute risk of a population. Despite the issues with the risk functions, this thesis provides a verified data set that is a necessary step for injury risk function evaluation. 


\section{TABLE OF CONTENTS}

ABSTRACT

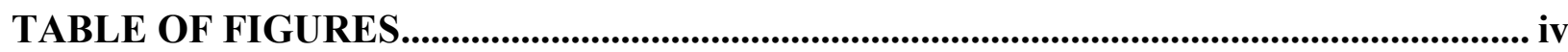

TABLE OF TABLES..................................................................................................... vi

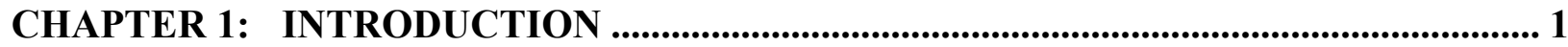

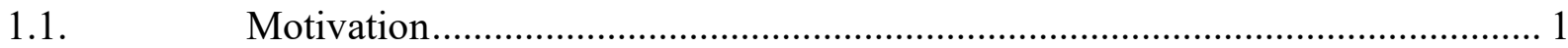

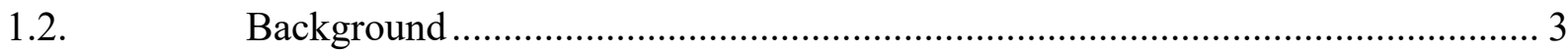

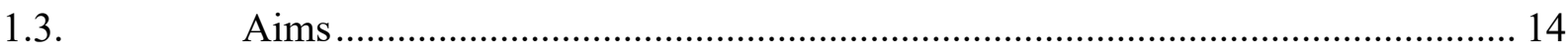

CHAPTER 2: HEAD BIOMECHANICS IN HUMANS ...................................................... 16

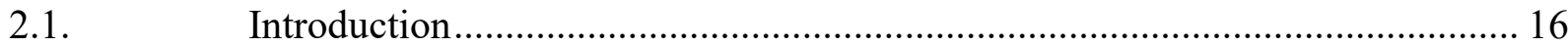

2.2. Literature review of human head kinematics.................................................. 16

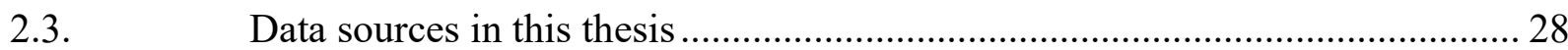

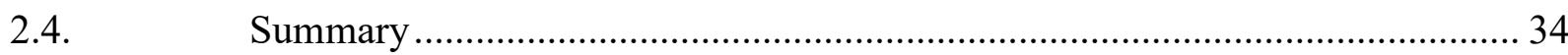

CHAPTER 3: ESTABLISHING A CONSISTENT DATASET ................................................ 36

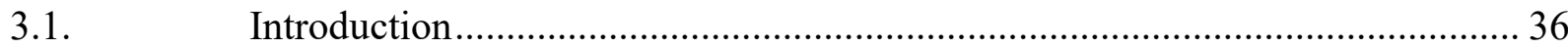

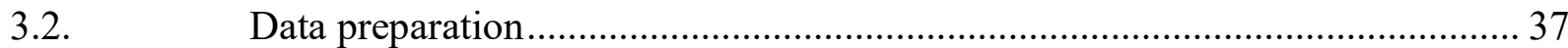

3.3. Kinematics-based injury risk metrics and injury risk functions ........................ 44

3.4. Strain-based injury criteria and injury probability predictions ........................... 51

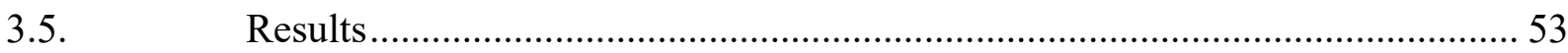

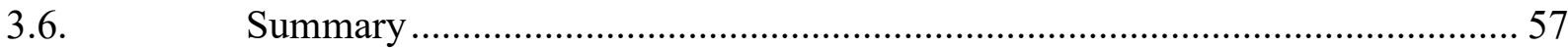

CHAPTER 4： EVALUATION OF CURRENT INJURY RISK FUNCTIONS................... 59

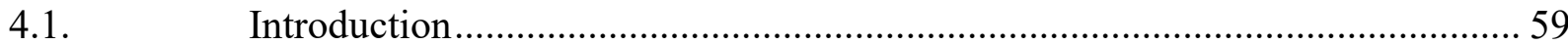

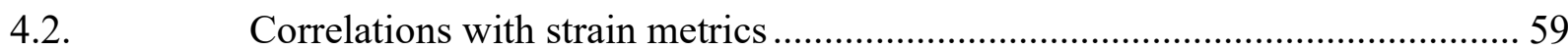

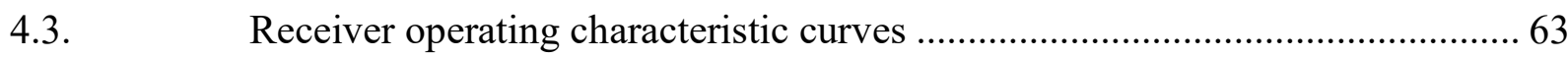

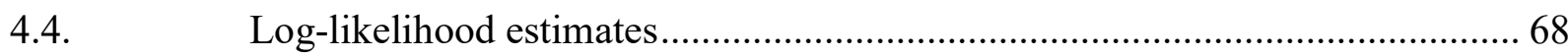

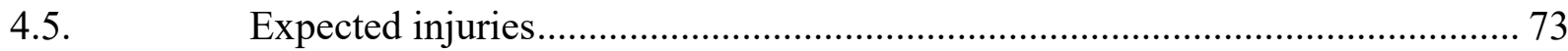

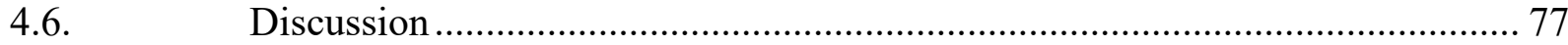

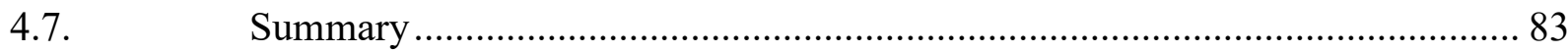

CHAPTER 5: CASE-CONTROL DATA AND LOGISTIC REGRESSION ....................... 84

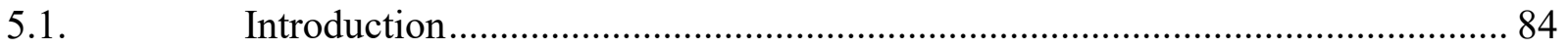

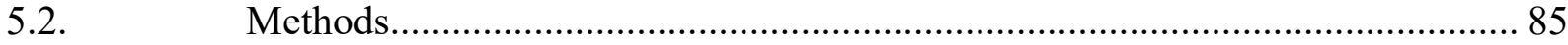

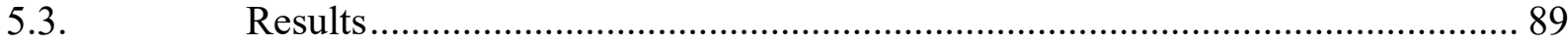




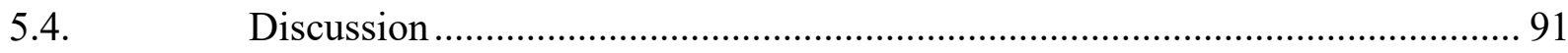

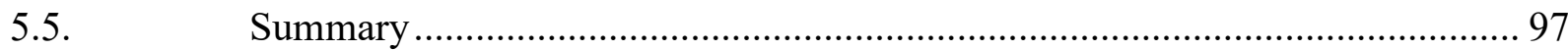

CHAPTER 6: CONCLUSIONS ................................................................................. 99

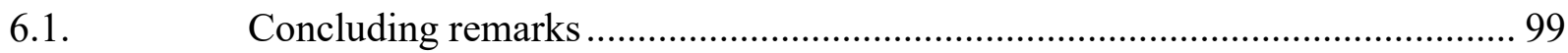

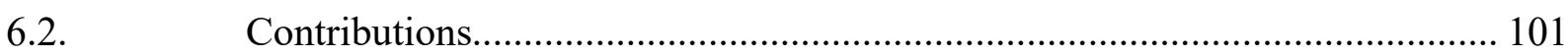

6.3. Future research directions ................................................................. 101

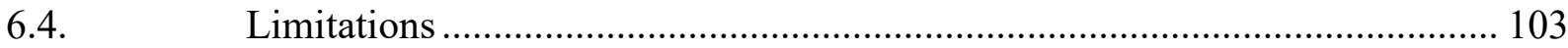

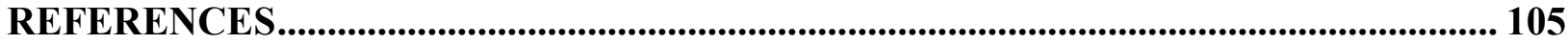

APPENDIX A: CONSISTENT AND INCONSISTENT NAP ARRAYS ........................... 126 APPENDIX B: DISTRIBUTION OF KINEMATIC AND STRAIN-BASED INJURY RISK PREDICTIONS ..................................................................................... 128 


\section{TABLE OF FIGURES}

Figure 1. Acceleration-duration tolerance curve for forehead impact to a hard, flat surface developed by Wayne State (adapted from Patrick et al., 1963)............................................. 6

Figure 2. Flowchart of inclusion criteria to assist in the selection of data sources........................ 30

Figure 3. Volunteer sled test configurations for (A) frontal, (B) oblique, and (C) lateral............ 32

Figure 4. Local head anatomic coordinate system with orientations defined according to SAE $\mathrm{J} 211$.

Figure 5. Chronological ordering of reconstructions showed that a common sensor was identified as inconsistent in subsets of the test series.

Figure 6. Change in distribution of the resultant head kinematics as a result of the corrections made to the professional football reconstruction data.

Figure 7. Linear displacement trajectories for each impact condition in the $\mathrm{XY}$ anatomical plane. Trajectories are plotted with respect to a coordinate system attached to the seat. The photo target indicates the location of the head CG.

Figure 8. Mid sagittal plane view of the FE human head models used in this thesis................... 52

Figure 9. Distribution of MPS over all elements from the FE model and strain metrics calculated from the distribution. 52

Figure 10. Distribution of maximum resultant kinematic parameters based on the 443 head impacts.

Figure 11. Distributions of the probabilities of injuries calculated from curves in Takhounts et al. using SIMon strain metrics as the predictor variable. 58

Figure 12. Distribution of strain metric values calculated via the GHBMC simulations..............58

Figure 13. Overall correlation between strain metrics from GHBMC and injury risk probabilities from kinematic- and strain-based metrics.

Figure 14. ROC curves (top) and their respective area under the curves (bottom) them of the injury risk probabilities estimated for various injury risk metrics

Figure 15. Plot of the negative log-likelihood estimates. Injurious cases (red line) that have a low probability of injury will have high values. The same is true with non-injurious cases (green line) that have a high risk of injury.

Figure 16. Negative log-likelihood estimates of all injury risk functions relative to 20 concussions. 71

Figure 17. Expected number of AIS 2+ and concussive injuries predicted using each of the injury risk functions calculated using the full dataset $(n=443)$. The full dataset had 31 observed concussions. 
Figure 18. Expected number of injuries predicted for each injury risk function using only the most severe tests for each volunteer rather than all $335 \mathrm{HRV}$ sled tests. The full dataset still

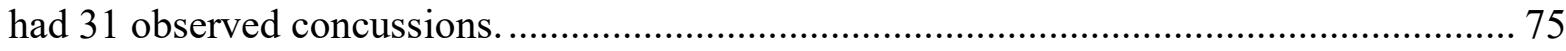

Figure 19. Injury risk function ranks with respect to each evaluation metric............................... 79

Figure 20. Weibull distribution fitted to the resultant head linear acceleration exposure in

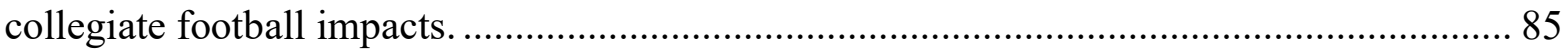

Figure 21. Plots of the original injury risk function developed by Funk et al. (2011) using a weibull distribution compared with its equivalent developed using logistic regression. Regression coefficients were chosen to mimize the difference between the two curves ....... 86

Figure 22. Exposure distribution used for sample scenarios B and C, in which only significant hits (i.e., above $35 \mathrm{~g}$ ) were considered. The Weibull exposure distribution is defined using the same coefficients as in Figure 20.

Figure 23. Distribution of the concussive and non-concussive impacts based on 10,000 samples.

Figure 24. Injury risk curves determined by the four sampling scenarios, compared to the curve

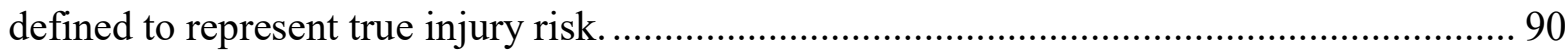

Figure 25. Two-by-two frequency table used for calculating the odds-ratio................................ 92

Figure 26. Definitions of a, b, c, and d from the frequency table (Figure 25), as they may apply to injury and non-injury distributions in football head impacts................................................. 93 


\section{TABLE OF TABLES}

Table 1. AIS injury severity descriptions ..................................................................... 4

Table 2. Summary of human head kinematic data sources used in this thesis. ........................ 35

Table 3. Inconsistent sensors in the NAP array and end times for each case. Blank rows under player 1 indicate helmet-to-ground impacts. Blank rows in the artefactual impact column

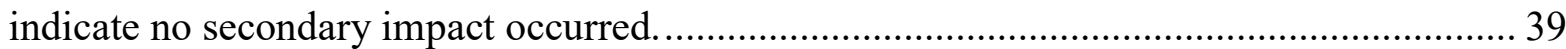

Table 4. Existing kinematics-based injury risk functions used in this thesis.............................. 50

Table 5. Strain-based injury risk functions used in this thesis................................................ 53

Table 6. Peak non-concussive injury metrics and probabilities and minimum concussive injury

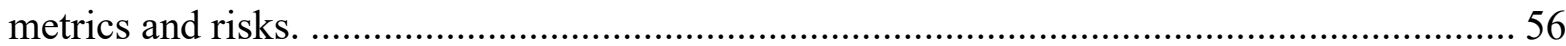

Table 7. Coefficients for the logistic regression models determined from each of the four sampling scenarios. Parentheses indicate $95 \%$ confidence intervals. 


\section{CHAPTER 1: INTRODUCTION}

\subsection{Motivation}

Significant advances have been made in reducing the number of serious and fatal injuries in the United States due to an improved understanding of injury biomechanics in humans. Notwithstanding the strides made in injury prevention, traumatic brain injury (TBI) remains a major global health problem (Taylor et al., 2017). An estimated 2.8 million emergency department visits, hospitalizations, and deaths resulted from TBI in the United States in 2013 (Taylor et al., 2017); falls (47\%), being struck by or against an object (15\%), and motor vehicle collisions (14\%) were the largest contributors (Taylor et al., 2017). In the military, approximately 320,000 soldiers have suffered from TBI since 2000 likely due to extended deployments and an increased use of improvised explosive devices (Pascrell, 2009). Sportsrelated concussions are also a major health concern, with an estimated 3.8 million concussions believed to occur annually due to sporting activities, although half of these are believed to be unreported (Harmon et al., 2013). When only considering children and adolescents under the age of 19 , the rate of emergency department visits for sports- and recreation-related concussions more than doubled between 2001 and 2009 (J. Gilchrist et al., 2011).

While head and brain injury related fatalities have decreased from federal regulations and protective equipment, the number of survivable TBIs spanning mild to severe type injuries seem to be rising (Coronado et al., 2015; Takhounts et al. 2013; Taylor et al., 2017). Several reasons have been postulated for this increase, including and increased awareness of concussion, improved reporting practices, a broadened definition of mild TBI (concussion) to include more symptoms, and better tools for clinical diagnosis (Coronado et al., 2015; Hootman et al., 2007; Taylor et al., 2017). Regardless, these trends necessitate better understanding of the relationship 
between head kinematics, brain biomechanics, and subsequent neuropathology, as well as the development of countermeasures and head protective equipment that can better prevent TBI.

Over the years, researchers have developed countless tools in an attempt to elucidate the relationship between head kinematics and brain injury. Tissue level parameters, such as intracranial pressure, brain strain, strain energy, and strain rate, von Mises stress, and more have been proposed as predictors of injury (Giordano \& Kleiven, 2014; Kleiven, 2007; Versace, 1971; Zhang et al., 2004), as well as various combinations rotational and translational head kinematics (Gadd, 1962; Kimpara et al., 2011; Newman, 1986; Newman et al., 2000a; S. Rowson et al., 2012). In each of these studies, however, one thing has remained constant: the need for human data to develop and validate these assessment tools.

Ommaya (1985) proposed concussion tolerance criteria, but indicated that these were highly speculative as they were based on primate data. This has been reiterated by others who have noted that little human data have been used to develop injury assessment and that the sample of specimens used may not be representative of the general population (Kimpara \& Iwamoto, 2012; S. Rowson et al., 2012). Others note that experimental data is currently lacking to properly validate these assessment tools, but say that if it becomes available these data should be used to revise the injury tools and tolerances (Sanchez et al., 2017a; Takhounts et al., 2011). This thesis aims to establish a verified set of human kinematic data and injury outcomes as a necessary step to develop and evaluate brain injury assessment tools. Then, the dataset will be used to begin to assess various injury risk functions. 


\subsection{Background}

\subsubsection{Types of brain injury and the Abbreviated Injury Scale}

The Centers for Disease Control and Prevention defines TBI as a disruption in the brain's normal function caused by a bump, blow, or jolt to the head, or by a penetrating head injury (Centers for Disease Control and Prevention, 2015; Marr \& Coronado, 2004). More specifically, TBI has been defined as an occurrence of head injury with at least one of the following: a decreased level of consciousness, amnesia, skull fracture, objective neurological or neuropsychological abnormality, a diagnosed intracranial lesion, or as an occurrence of death resulting from head trauma (Thurman et al., 1995).

TBIs can be broadly classified as either focal-type or diffusion type injuries. Focal type injuries are associated with localized damage visible to the naked eye. These are typically induced when an object penetrates the skull, such as the result of a motor vehicle crash, and include cerebral contusion and laceration, and epidural, subdural, and intracerebral hemorrhage (LaPlaca et al., 2007). Diffuse brain injuries are associated with more widespread disruption of brain tissue and microscopic damage (Adams et al., 1977; Gennarelli, 2005). Diffuse type brain injuries form a spectrum of injuries; the most severe form is diffuse axonal injury (DAI). DAI is the widespread damage to the brain's white matter; symptoms include the immediate loss of consciousness lasting for days to weeks (Gennarelli et al., 1982).

The most common type of TBI is mild diffuse brain injury, more commonly known as concussion (Centers for Disease Control and Prevention, 2003). The 2012 Zurich Consensus statement defines concussion as "a complex pathophysiological process affecting the brain, induced by biomechanical forces" (McCrory et al., 2013). Symptoms include headache, feeling in a fog, emotional instability, loss of consciousness, amnesia, irritability, slowed reaction times, 
or insomnia (McCrory et al., 2013). Guidelines were also set forth for diagnosing concussions, including the use of signs, symptoms, computer-based neurocognitive testing, and clinical judgment (McCrory et al., 2013).

Head and brain injuries, such as concussion and DAI, can also be classified using the Abbreviated Injury Scale (AIS). AIS is an anatomically-based coding system used to classify and describe injury severities according to the threat to life associated with the injury (Gennarelli \& Wodzin, 2008). Injuries are ranked on a scale from 1 to 6 , with levels 1 to 5 indicating mild to severe injuries and 6 representing a fatal injury (Table 1). Skull fractures can be rated as an AIS 2, AIS 3, or AIS 4 injury depending on the type and location of fracture; a contusion is generally an AIS 3 injury and DAI is classified as AIS 4+ (Gennarelli \& Wodzin, 2008). Concussion has several associated AIS severities depending on the both brain region, loss of consciousness, and other factors; previous research has used a general definition of concussion as an AIS 2+ injury (Takhounts et al., 2013) or as an AIS 2-3 injury (Viano, Parenteau, Xu, \& Faul, 2017). This thesis follows the approach by Takhounts et al., in that concussion is assumed to be an AIS 2+ injury for the injury risk functions in later chapters.

Table 1. AIS injury severity descriptions

\begin{tabular}{ccr} 
AIS & Injury & Concussion Symptoms \\
Code & Severity & \\
\hline AIS 2 & Moderate & Mild-to-moderate concussion with loss of consciousness $<1 \mathrm{~h}$ \\
AIS 3 & Serious & Severe concussion with loss of consciousness 1-6h \\
AIS 4 & Severe & Diffuse axonal injury with loss of consciousness $6-24 \mathrm{~h}$ \\
\hline
\end{tabular}




\subsubsection{Brain injury criteria}

Brain injury criteria provide a means for assessing brain injury risk during head impact or inertial loading. Traditionally, injury criteria were developed for interpreting anthropometric test device (ATD) responses in vehicle safety assessments, but their use has since been extended to other impact environments such as sport and military. Brain injury criteria consist of two parts: a biomechanical metric and an injury risk function. Numerous brain injury metrics have been proposed to relate engineering parameters, such as head kinematics or tissue-level strains, to head impact severity; the injury risk curve then relates this metric to a likelihood of brain injury via a probabilistic function.

Traditionally, head injury risk metrics have been based on translational kinematics (Gadd, 1962; Lissner et al., 1960; Versace, 1971). Maximum resultant linear acceleration was found to correlate with skull fracture (Gurdjian et al., 1966; Lissner et al., 1960); this correlation led to the development of the Wayne State Tolerance Curve (WSTC), which defines a skull fracture threshold based on resultant linear head acceleration and impact duration (Figure 1; Patrick et al., 1963). Gadd (1962) fitted data from the WSTC in order to develop the Severity Index (SI). Later a time averaging component was added such that SI became the Head Injury Criterion (HIC; Versace, 1971). Currently, HIC is the only federally mandated safety standard in automobile design (NHTSA, 2000); SI and peak linear acceleration have also been used in helmet safety evaluations and regulations (NOCSAE, 2015; S. Rowson \& Duma, 2011). 


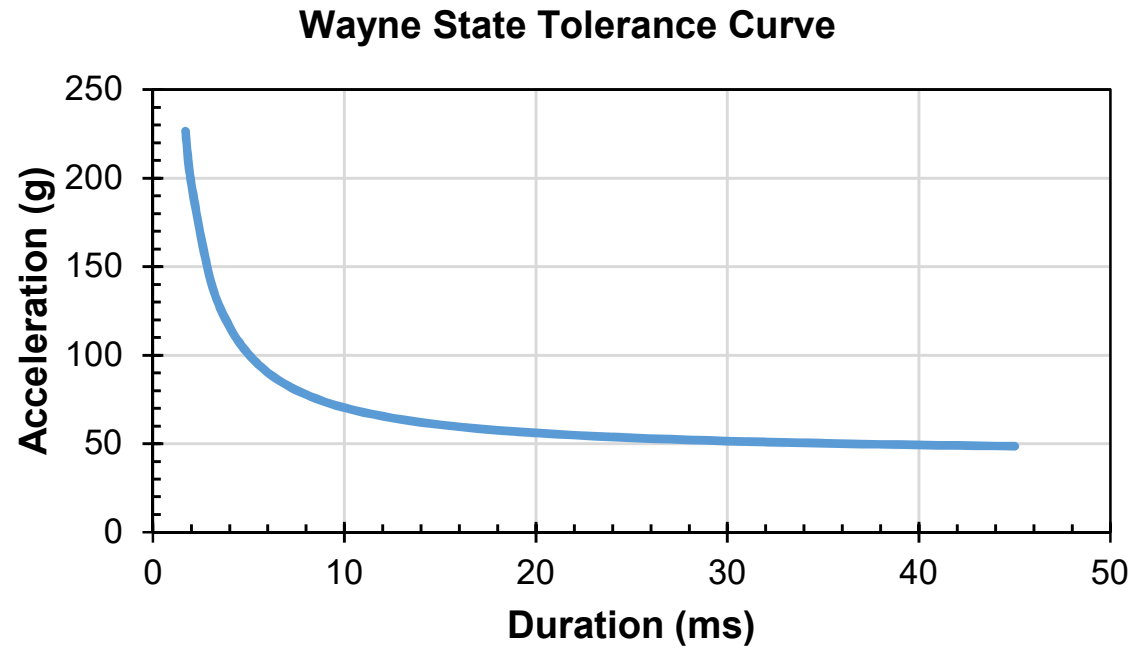

Figure 1. Acceleration-duration tolerance curve for forehead impact to a hard, flat surface developed by Wayne State (adapted from Patrick et al., 1963).

Despite the widespread use of translational-based metrics, some have questioned whether they can assess brain injury from closed-head impacts (Gabler et al., 2016a; Gennarelli et al., 1982; Margulies \& Thibault, 1992; Takhounts et al., 2013). Holbourn, (1943) proposed rotational head motion as a better predictor for brain strains, contending that shear strains are the primary mechanism of diffuse type brain injuries and that rotation is the simplest way to generate shear strain in the brain. Studies have developed metrics and tolerances based on rotational kinematics, such as the Brain Injury Criterion (BrIC), which was developed to serve as a complement to HIC in the automotive industry, and the Rotational Injury Criterion (RIC) (Kimpara \& Iwamoto, 2012; S. Rowson et al., 2012; Takhounts et al., 2013). Combinations of translational and rotational kinematics have also been proposed as risk assessment metrics (Newman, 1986; Newman et al., 2000a; S. Rowson \& Duma, 2013).

Finite element (FE) models are the current state of the art for studying the mechanical response of the brain during head impact. Validated FE models have provided a useful alternative for studying brain deformation given that direct measures of tissue-level brain 
mechanics are challenging to collect (Mao et al., 2013; Takhounts et al., 2008). In literature, FE models have been used to simulate real-world events, with results of these simulations assisting in the derivation of tissue-based injury metrics to categorize brain tissue injury (Bandak et al., 2001; Gabler et al., 2016b; Kleiven, 2007; Marjoux et al., 2008; Panzer et al., 2012; Takhounts et al., 2003, 2008, 2011, 2013; Zhang et al., 2004). Examples of the tissue-based metrics include maximum principal strain (MPS; Kleiven, 2007), von Mises stress (Deck \& Willinger, 2008), cumulative strain damage measure (CSDM; Takhounts et al., 2003), and tract-oriented strain (TOS; Sullivan et al., 2015).

\subsubsection{Injury risk functions}

Regardless of the predictor variable used to assess injury risk, the variables must be able to accurately predict injury over a broad range of severities. A single tolerance is often used for the purpose of safety standards; for example, a maximum HIC value of 700 is allowed in vehicle regulations (Eppinger et al., 1999), while helmet regulations limit the severity index to less than 1200 (NOCSAE, 2015). While these values are generally chosen based on the probability of injury at that threshold, a single value does not account for the variation in human tolerance due to factors such as genetic predisposition, age, and gender. Thus enters the necessity for the injury risk function, a tool which determines the probability of injury for a given impact rather than a simple pass or fail. Kent \& Funk (2004) state that a fundamental purpose of the field of injury biomechanics is to develop risk curves defining injury probability as a function of known predictors. Generally, injury risk functions are expressed as a probabilistic curve given that the likelihood of injury is dependent on many factors, such as age, gender, physiologic condition, and other factors (Crandall et al., 2011). 
A common approach for developing injury risk functions is to assume a form of the function and optimize the fit of the assumed form such that estimated outcomes match observations. Literature has shown four common statistical distributions used to define the functional form of the injury risk function: log-normal, logistic, Weibull, and normal distributions. Logistic regression is perhaps the most common form for deriving injury risk curves from biomechanical data due to the ease of use for modelling binary responses (i.e. injured and uninjured), although the other distributions are also capable of modelling binary response data (DiDomenico \& Nusholtz, 2003; Kent \& Funk, 2004)

\subsubsection{Data sources for injury risk functions}

As mentioned above, the purpose of an injury risk function is to relate biomechanical variables to a probability of injury in order to better develop and evaluate the protective capabilities of equipment and countermeasures. Designers of head safety equipment and automotive injury countermeasures use the risk assessment models to drive design, thus they require accurate assessment models that can predict brain injury over a wide range of severities. However, a paradox arises because while this equipment is designed to protect human subjects, human research volunteers cannot be used in laboratory tests that may jeopardize their wellbeing. Researchers must therefore conduct experiments on human surrogates and extrapolate the results to human subjects in order glean as much information as possible without injuring volunteers.

Early research primarily focused on quantifying risk for open-type head injuries like skull fracture. As such, post-mortem human subjects (PMHS) have been used at length to study tolerance and injury risk to impacts for skull fracture. Prasad \& Mertz (1985) developed the injury risk functions for HIC using skull fracture data collected from PMHS head drop tests 
(helmeted and unhelmeted) and impacts against windshields. These curves were expanded to represent the cumulative injury probability at various injury AIS levels by extending the relationship between maximum AIS 3 and 4 curves for Thoracic Trauma Index (NHTSA, 1995); the resulting curves represent cumulative injury probability at a given HIC level (AIS $1+$ to AIS $5+$ ). For example, a HIC value of 1000 represents a $17 \%$ probability of severe head injury (AIS $4+$ ), a $53 \%$ probability of serious injury (AIS $3+$ ) and an $89 \%$ probability of moderate head injury (AIS 2+; NHTSA, 1995). In automobile design, a maximum HIC value of 700, or a $6 \%$ probability of AIS 4+ injury, is allowed in automobile design under the provisions of the U.S. advanced airbag regulation (NHTSA, 2000).

$\mathrm{HIC}$ and its injury risk curve were designed using skull fracture data; as such, HIC has been successful in mitigating severe head injuries, but it does not address mild TBI such as concussion. Further, skull fractures can be identified in PMHS experiments, but this type of human surrogate lacks the physiologic response needed to diagnose brain injuries. Animals, on the other hand, provide a viable surrogate to study physiologic response to injury, and have been used at length to determine brain injury tolerances. Early research on rotational brain injury was pioneered by Ommaya et al. (1964), who derived injury tolerances from primates. Since then, studies have generally used primates and pigs as surrogates for human brain injury response (Abel et al., 1978; Margulies et al., 1999; Meaney et al., 1993; Ommaya, 1985; Stalnaker et al., 1977). Data from these studies have become the basis for modern brain injury criteria; for example, Takhounts et al. $(2011,2013)$ developed injury risk functions for two DAI based strain injury metrics (MPS and CSDM) from animal data by applying scaled kinematic loading conditions to an FE model and relating the model results to the animal injury outcomes. 
Correlations between these strain metrics and the kinematic metric BrIC were also determined by Takhounts et al. (2013) in order to develop injury risk functions for BrIC.

A more recent approach has been to collect data from sensors worn by contact sport athletes so that clinical injury outcomes can be related to engineering parameters to develop injury risk functions. Researchers have used various types of sensor-mounted headgear (Chamouard et al., 1987; Duma et al., 2005). instrumented mouth guards (Camarillo et al., 2013; D. A. King et al., 2015; Siegmund et al., 2014), and skin patches (Press \& Rowson, 2017; Reynolds et al., 2016; Stucker, 2015) worn by athletes to collect kinematic data during the sporting event which can then be related to clinical injury outcomes. The advantage of these types of sensors is that they directly measure head kinematics resulting from impact. Additionally, wearable sensors collect all impacts above a specified threshold, providing information about the exposure various levels of head impacts (Altman, 1990; Bonita et al., 2006; Duma et al., 2005; Funk et al., 2007). Both the kinematic measures and the exposure data have been used to develop injury risk functions; for example, Rowson et al. (2012) and Rowson \& Duma (2013) employed a helmet-mounted sensor array, known as the Helmet Impact Telemetry (HIT) system, to develop injury risk functions from collegiate football impacts for several predictor variables.

Another way to relate head kinematics and clinically diagnosed injuries is by recreating head injuries in real-life impacts. In literature, reconstructions have been conducted computationally and experimentally using various inputs, such as impact speed found via an event data recorder, eye witness account, or video analysis. For example, computational simulations have been used to reconstruct head injuries seen from falls, motor vehicle crashes, and pedestrian impacts (Auer et al., 2001; O’Riordain et al., 2003; Somers et al., 2011). 
Laboratory reconstructions, on the other hand, have been used to reconstruct impact events in sports in order to glean insight to the head kinematics that may not have been measured via wearable sensors (Begeman \& Melvin, 2002; Fréchède \& McIntosh, 2009; McIntosh et al., 2000; Pellman et al., 2003). Newman et al. (2000a, 2005) and Pellman et al. (2003) reconstructed professional football, concussive impacts in the late 1990s; the resulting data have been to develop injury risk functions for various kinematic metrics (Newman et al., 2000a; Pellman et al., 2003).

\subsubsection{Limitations of the data used for injury risk function development}

While the data sources mentioned above are the best available for studying human tolerance to brain injury, each is not without limitations. PMHS are an exact representation of human anatomical structures, but they lack the tissue properties and physiologic response needed to produce comparable injuries and responses to humans (Crandall et al., 2011). This is especially true for concussions, which are generally only able to be diagnosed via functional symptoms (McCrory et al., 2013). Animal models have been used to study virtually every body region, however the interpretation of animal results is complicated by differences in anatomy and physiology (Crandall et al., 2011; Kent \& Bass, 2007). Clinically-relevant, functional outcomes from animal data are contingent upon using appropriate techniques to scale and extrapolate the original data to human injury outcomes; this is an area that remains an open research question (Crandall et al., 2011; Ommaya, 1985; Panzer et al., 2014).

Human research volunteers (HRVs) eliminate the scaling question as they are identical to the population of interest. At the same time, they have the obvious drawback in that laboratory tests must be performed at sub-injurious levels (Belmont Report, 1979; Declaration of Helsinki, 1996, "The Nuremberg Code," 1949). As such, injury risk functions cannot be derived solely 
using data collected from HRVs, but must be developed in conjunction with other, injurious data. Research volunteer data can be used, however, to assess whether injury risk functions are over predictive, although determining the accuracy of a risk function requires additional assessments using higher severity head impact data (Sanchez et al., 2017a).

Wearable sensors have been deployed to collect potentially injurious human data, but technical challenges appear to limit the accuracy of their kinematic measures in certain impact conditions. For example, helmet fit and padding in instrumented headgear may affect sensor coupling to the human head and cause errors in measurement if there is relative motion between the helmet and head (Beckwith et al., 2012; Jadischke et al., 2013; Siegmund et al., 2015). Similarly, instrumented mouth guards are dependent on fit and wear, as well as factors such as mandible motion, variation is fabrication, and unanticipated impacts in which the jaw is open (Camarillo et al., 2013; Wu et al., 2016). Non-rigid skin coupling can lead to incorrect predictions of head motion for both skin patches and skull cap sensors, with studies reporting kinematic artifacts resulting from soft-tissue dynamics (Lucchetti et al., 1998; Shultz et al., 2011; Wu et al., 2016).

Injury reconstructions allow researchers to relate clinical injury diagnoses to quantifiable engineering parameters, but unlike the wearable sensors, they can only provide an indirect measure of the engineering parameters. Additionally, a substantial amount of uncertainty exists from the reconstruction process. For example, error sources may stem from the methodology used to determine reconstruction initial conditions, the use of a human surrogate (ATD or computational model), the sensors mounted in an ATD, and the material properties or boundary conditions in a computational model (M. D. Gilchrist \& Doorly, 2009; Newman et al., 2005; 
O'Riordain et al., 2003). Computational requirements could also limit the use of computational modeling (Doorly et al., 2005a).

\subsubsection{The necessity of human data for risk functions}

Despite the limitations, the underlying need for human biomechanical data for the development and validation of injury assessment tools and injury risk functions has been

reiterated throughout literature (Ommaya, 1985; Sanchez et al., 2017a; Takhounts et al., 2011). Additionally, while FE models of the human brain are theoretically well-suited for studying the tissue-level response of a head impact due to their capacity of predicting strains based on head motion (Gabler et al., 2016a; Takhounts et al., 2013; Zhao et al., 2017), different state-of-the-art head and brain models can predict widely different strain responses for the same kinematic inputs of the head (Ji et al., 2014). Reliable, biomechanical head response and injury data are needed to improve both model validation and predictive capabilities.

By combining the kinematic and injury data from wearable sensors, reconstructions, and HRVs, it may be possible to address some of the limitations associated with each. HRVs have reliable biomechanics, but no injury, while wearable sensors have injury outcomes, but questionable biomechanics and reconstructions have both injury outcomes and good biomechanics, but questionable accuracy. Combining these three sources of human data may provide the biomechanical information needed to assess injury risk functions. Accurate brain injury risk assessment models can have a significant effect on the development of head safety systems including helmets and vehicle restraints; thus, the accuracy of these metrics in terms of correctly predicting brain injury over range of severities is of utmost importance. 


\subsection{Aims}

\subsubsection{Objectives}

The first goal of this thesis is to establish a consistent database of human head kinematic data of concussive and non-concussive impacts. Consistent is be defined such that injuries are the same for all cases (i.e., concussions only) and that all kinematic data are valid representations of the measured motion and are processed in the same manner. Chapter 2 consists of a literature review of previously collected direct and indirect measures of human head kinematics, all of which could serve as potential data sources. Datasets were chosen from these sources in order to accomplish the second goal of this thesis: to evaluate the capability of injury risk functions to predict injury outcomes.

A primary goal of the field of injury biomechanics is to develop injury risk assessment models that can accurately assess injury probabilities using one or more predictor variables. Various injury metrics and risk functions have been proposed with little consensus on which should be used, therefore evaluation is needed to determine which risk function performs best for various loading and impact conditions. The second objective of this thesis is to assess injury risk functions according to their ability to correctly predict injuries over the entire dataset. Efforts were also made to address the certain limitations of the injury risk functions and their underlying data.

\subsubsection{Assumptions}

Diffuse brain injuries, such as concussion and DAI, are believed to be caused by brain deformation (Gennarelli et al., 1982). Therefore, in his thesis, strain-based brain injury metrics (MPS and CSDM) are assumed to be representative of diffuse brain injuries in closed-head impacts. It should be noted, however, that various other mechanical variables have been 
proposed as predictors of brain injury. Many early studies focused on intracranial pressure as the mechanism of brain injury (Nahum et al., 1977); this theory has been revisited in present day (Zhao \& Ji, 2016). Strain rate, the product of strain and strain rate, and stress have also been proposed as mechanisms of brain injury in the literature, although the results of experimental studies have been mixed (Cater et al., 2006; Morrison et al., 2003). 


\section{CHAPTER 2: HEAD BIOMECHANICS IN HUMANS}

\subsection{Introduction}

The research field of injury biomechanics works to identify injury mechanisms and define injury tolerances in humans (Viano et al., 1989). Historically, to achieve this endeavor, several types of human surrogates and manners of data collection have been used. Laboratory studies have recruited human volunteer subjects to participate in various experiments ranging from everyday activities to more vigorous events. While laboratory experiments cannot be designed to cause harm to the subject, wearable sensors have been employed on contact sport athletes to collect head kinematics and relate them with potentially injurious outcomes. Researchers have also tried to indirectly measure head kinematics associated with injurious and non-injurious impacts by recreating these events in a laboratory environment or using a computational model. This chapter provides an overview of the literature that has used these methods to capture human head biomechanics resulting from impact.

\subsection{Literature review of human head kinematics}

\subsubsection{Laboratory studies of everyday activities}

Several studies have measured head motion during everyday activities in a laboratory setting (Allen et al., 1994; Arndt et al., 2004; Bussone \& Duma, 2010; Ng et al., 2006; Vijayakumar et al., 2006). Activities included hopping, plopping into a chair, a head turn, coughing, and more. Generally, these events were characterized as having no direct impact, a relatively short time duration, and low accelerations. They also had a small probability of injury and results were reported as peak kinematic parameters. For example, studies found that passively falling into a chair yielded linear accelerations between 3.7 and $10.1 \mathrm{~g}$ and angular 
accelerations between 129.1 and $169 \mathrm{rad} / \mathrm{s}^{2}$ (Allen et al., 1994; Bussone \& Duma, 2010; Funk et al., 2011; Vijayakumar et al., 2006). Other results reported that jumping off a step caused linear and angular accelerations up to $8.1 \mathrm{~g}$ and $206.4 \mathrm{rad} / \mathrm{s}^{2}$, respectively (Allen et al., 1994; Bussone \& Duma, 2010; Funk et al., 2011; Ng et al., 2006), and pogo stick hopping resulted in a linear acceleration of $4.5 \mathrm{~g}$ (Arndt et al., 2004).

Every day activities that involve a direct impact to the head have also been studied. Arndt et al. (2004) measured the maximum acceleration from various activities such as a pillow strike. They found a maximum linear acceleration from the pillow strike of $28.1 \mathrm{~g}$ and a duration of $0.1 \mathrm{~s}$ (Arndt et al., 2004). This acceleration is similar to that found by Funk et al. (2011) due to a soccer ball impact at $11.5 \mathrm{~m} / \mathrm{s}(21 \pm 4.9 \mathrm{~g})$. Symptoms reported after the soccer ball include tightness of neck and stinging of the forehead, but none lasted for longer than one day and subjects were considered uninjured.

\subsubsection{Human volunteer sled tests}

Human volunteers have also participated in studies at slightly higher severities than those reported by the everyday activities studies. The data gathered in these studies have been used at length for the development and validation of test dummies. Weis et al. (1963) studied a number of human exposures to a vertical drop, however instrumentation was mounted on the rigid vehicle and not the volunteer as was typical in a majority of the pre-1967 human subjects research. When volunteers were instrumented, generally the head was restrained or not instrumented; in the cases that there were head-mounted sensors, limitations on sensor technology (e.g. noise, data spikes) made these results unusable (Ewing \& Thomas, 1972; Stapp \& Taylor, 1964; Stapp, 1951). 
The Naval Biodynamics Laboratory (NBDL) conducted volunteer subject sled tests in the late 1960s to study the effect of head and neck postures, impulse direction, and impulse magnitude on the kinematic head and neck response (Ewing et al., 1969, 1975, 1976, 1977, 1978; Ewing \& Thomas, 1972). Subjects were exposed to sled pulses in various configurations at $1 \mathrm{~g}$ increments between $3 \mathrm{~g}$ and $16 \mathrm{~g}$. Different studies analyzed the effect of the rate of onset (Ewing et al., 1976), impact direction (Ewing et al., 1977, 1969), and initial head and neck position (Ewing et al., 1975, 1978). Medical examinations before and after each test reported no pathological changes due to the test series, although some subjects reported minor surface abrasions due to the restraint system (Ewing \& Thomas, 1972). All subjects returned to their usual duty and off-duty activities post-series (Ewing \& Thomas, 1972).

A similar study conducted by Hendler et al. (1974) exposed volunteers to increasing sled acceleration pulses in order to analyze the kinematic response to a simulated barrier crash impact as an consequence of initial positioning and muscle tensing. Other studies have conducted HRV sled tests with various acceleration pulses in order to study the effect of various restraints in a lateral impact configuration (Kumar et al., 2006; Zaborowski, 1964, 1965) and due to barrier crashes (Bohlin, 1964). Studies have also used sled tests to characterize and compare the occupant kinematics of volunteers, PMHS, and ATDs by assessing the excursion seen by each group (Beeman et al., 2012) and to characterize the effect of bracing on the kinematics (Beeman et al., 2011; Olafsdottir et al., 2013).

Arbogast et al. (2009) conducted a series of non-injurious, frontal sled tests in order to quantify and compare the kinematic responses of a child to those of an adult. Pediatric (6-15 years old) and adult (18-40 years old) volunteers were restrained with a three-point belt and exposed to a sled pulse of $4.9 \mathrm{~g}$, similar to a bumper-car impact. The researchers normalized the 
head trajectory data in order to determine factors governing the differences seen between the two groups of HRVs (Arbogast et al., 2009). A later study also conducted by Arbogast et al. (2012) used pediatric and adult male subjects in far-side lateral and oblique sled test configurations to study the effect of seatbelt pretensioning across age groups.

The data collected in the studies in this section and the previous section were necessary for documenting the head response of ordinary people engaged in everyday and more vigorous types of physical activities. The data have been used to establish a range of common head loading and kinematics for use with ATD validation (Beeman et al., 2012; Seeman et al., 1986; Wismans \& Spenny, 1984) as well as presenting kinematic information that presents a minimal risk of injury, which can be compared to head kinematics in different loading environments such as roller coaster rides (Smith \& Meaney, 2002). In terms of injury risk functions, however, these data can only be used to develop and assess the lower end of injury risk functions. Higher severity impacts as well as injuries are needed for the upper end of the injury risk curves. The following sections outline data that fall into this category of higher severity and potentially injurious data.

\subsubsection{The use of the HIT system in contact sports}

Contact sport athletes comprise a group of human subjects who voluntarily expose themselves to impacts during the course of their sporting events. These impacts have the possibility of being injurious, therefore, wearable sensors have been developed to capture the head kinematics during such events and relate them to injury outcome. One type of wearable sensors is instrumented helmets. Most researchers who have employed instrumented helmets have used a six-accelerometer, five degree-of-freedom (DOF) helmet-mounted sensor array known as the HIT system (Simbex, Lebanon, NH). Duma et al. (2005) measured head 
accelerations in collegiate football players using the HIT system during 2003 season, recording 3312 head impacts over 10 games and 35 practices from thirty-eight different players. The average peak head acceleration experienced for all impacts averaged $32 \pm 25 \mathrm{~g}$ (HIC: $26 \pm 62$; SI: $36 \pm 91$ ), although most impacts (89\%) resulted in a peak head acceleration below $60 \mathrm{~g}$ (Duma et al., 2005). The distribution of peak angular acceleration was also skewed left, and averaged 905 $\pm 1075 \mathrm{rad} / \mathrm{s}^{2}$ about the $\mathrm{X}$-axis and $2020 \pm 2042 \mathrm{rad} / \mathrm{s}^{2}$ about the $\mathrm{Y}$-axis. Only one of these measured impacts caused a concussion $\left(81 \mathrm{~g}, 200 \mathrm{HIC}, 5600 \mathrm{rad} / \mathrm{s}^{2}\right.$ about the $\mathrm{X}$-axis, $5590 \mathrm{rad} / \mathrm{s}^{2}$ about the $\mathrm{Y}$-axis).

The goal of this study focused on describing the exposure to impact as well as characterizing peak acceleration, impact direction, and impact duration; other studies had similar aims (Brolinson et al., 2006; Duma et al., 2005; S. Rowson et al., 2009). Studies have also implemented the HIT system in order to examine differences in head impact exposure and magnitude as a result of player position and session type (i.e. practice versus game). Crisco et al. (2011) collected data over three seasons from three collegiate football teams and found that running backs and quarter backs received the highest magnitude of impacts, while offensive and defensive linemen and line backers had a higher frequency of impacts. High school football players showed similar trends, namely, defensive linemen and offensive skill players had higher head accelerations due to impact whereas the line players received the greatest number of impacts (Broglio et al., 2009, 2011a, 2013; Schnebel et al., 2007). More recently, youth football players have become the focus of this type of study (Cobb et al., 2013; Daniel et al., 2012; Young et al., 2014).

Given the desire to determine biomechanical thresholds for concussion, studies have also attempted to find relationships between head kinematics and clinical diagnoses using the HIT 
system data. A study of high school football players attempted to identify relationships between symptoms and cognitive performance change scores to impact biomechanics measured by the HIT system, but was unable to find any significant associations from 20 diagnosed concussions (Broglio et al., 2011b). Guskiewicz et al., (2007) conducted a similar study at the University of North Carolina at Chapel Hill which attempted to relate impact characteristics (i.e. magnitude and location) to changes in symptom severity, postural stability, and neurocognitive function compared to baseline values. Thirteen concussive impacts were measured, but no relationship was found; the authors concluded that concussive symptoms seemed to be largely independent of impact characteristics (Guskiewicz et al., 2007).

McCaffrey et al. (2007) assessed symptom presence and severity, neuro-cognitive performance, and postural stability after low-impact (defined as less than $60 \mathrm{~g}$ ) and high-impact ( $>60 \mathrm{~g}$ ) test sessions. Impact severity was defined via the HIT system, and no deficits were found after either impact session when compared to baseline measures (McCaffrey et al., 2007). The HIT system has been used to show that repetitive sub-concussive impacts over a single season do not correlate with changes to neurologic impairment, nor does concussion history relate to changes in neurologic performance (Gysland et al., 2012). Studies employing the HIT system have also found that cumulative head impact burden, defined as impact volume and intensity, does not relate to concussion threshold (Broglio et al., 2011a; Eckner et al., 2011).

Beckwith et al. (2013b) found that concussions diagnosed immediately after the impact event were generally associated with higher kinematic measures recorded via HITS, whereas concussions with a delayed diagnosis were associated with a higher number of impacts; further, they showed that players sustained more impacts and impacts of higher severity levels on days with a concussion diagnosis than on days without a diagnosis (Beckwith et al., 2013a). 
Additionally, this study found that impact severity measures derived from peak linear acceleration were the best predictors of immediately diagnosed concussions (Beckwith et al., 2013a). Other studies have also aimed to find kinematic predictors of concussion using data from the HIT system. Greenwald et al. (2008) found that weighted combinations of biomechanical inputs served as a better predictor of concussion than a single measure. Rowson et al. (2012) published injury risk functions for concussion based on almost 310,000 collegiate football impacts using maximum resultant head angular acceleration and velocity as predictor variables; this study associated a $50 \%$ risk of concussion with $6383 \mathrm{rad} / \mathrm{s}^{2}$ and $28.3 \mathrm{rad} / \mathrm{s}$ for angular acceleration and angular velocity, respectively.

Clearly, a large amount of real-world kinematic data has been collected from football impacts at various levels using the HIT system. Researchers have started to expand use of the HIT system to other sports as well. For example, researchers fitted youth ice hockey players with instrumented helmets to measure game and practice impacts; they found these athletes regularly sustained head impacts with magnitudes of $20 \mathrm{~g}$, values similar in severity to college football players (Mihalik et al., 2008). Studies have also used HITS in ice hockey players to describe the frequency, magnitude, and direction of impacts as a factor of player positions and found greater impact magnitudes in games than practices, but little difference due to position (Mihalik et al., 2008, 2012). Differences in youth ice hockey impact characteristics have been studied in a variety of situations. For example, higher measures of head impact severity have been associated with infractions sustained during play when compared with non-infraction collisions, higher head liner and angular accelerations were observed in open-ice collisions as opposed those along the playing boards, and anticipated collisions tended to result in lower angular accelerations than unanticipated impacts (Mihalik et al., 2010a, 2010b). 
Researchers have also conducted studies using athletes from collegiate men's and women's ice hockey to characterize the head impact exposure and investigate differences due to sex, position, and session type. Wilcox et al. (2014a) found that male and female players experienced impacts of similar linear accelerations, but male athletes had higher peak angular acceleration values. Men also had a higher frequency of head impacts due to contact with another player or with the boards than females did, and the impacts had larger rotational accelerations (Wilcox et al., 2014b). Further, male ice hockey players tended to have a higher frequency of impacts, despite women having a higher frequency of concussion diagnoses (Brainard et al., 2012; Wilcox et al., 2014a).

While the HIT system has been a valuable tool in measuring and characterizing head impacts in sports, it has a few important limitations that should be mentioned. Literature has noted that the HIT system was evaluated using a medium-sized helmet on a Hybrid III dummy, but this is the incorrect size based on the manufacturer's fitting instructions; the correctly sized helmet had measurements errors greater than 15\% in most cases (Jadischke et al., 2013). Differences is measurement values are likely due to relative motion between the helmet and head (Beckwith et al., 2012; Lewis et al., 2001; Manoogian, McNeely, Duma, Brolinson, \& Greenwald, 2006). Additionally, the HIT system cannot directly measure head angular acceleration (Funk et al., 2007). Instead, peak angular acceleration is calculated from the linear acceleration measures (Crisco et al., 2011; S. Rowson et al., 2012). Angular velocity is also estimated, using the calculated values of peak resultant angular accelerations (S. Rowson et al., 2012). Some studies did a 12-accelerometer, 6 DOF in helmet accelerometer array which can measure rotational head motion. However, both this 6 DOF device and the HIT system only record 40ms of head impact data, which may not be enough time for the kinematics, namely 
angular velocity, to fully develop; angular velocity has been suggested as the best predictor of brain strain response (Gabler et al., 2016a; Takhounts et al., 2013). Further, the material properties of brain tissue cause the strain response to lag the head velocity (Chatelin et al., 2010; Mao et al., 2013; Takhounts et al., 2008), necessitating longer time histories for use in FE models.

\subsubsection{Other wearable sensors}

While the HIT system is possibly the most established wearable technology, it is not the only one utilized by researchers. Naunheim et al. (2000) mounted a triaxial accelerometer on the vertex of a helmet to measure and compare head impacts experienced by high school hockey, football, and soccer athletes. Soccer players heading a ball recorded the largest head accelerations $(54.7 \pm 4.1 \mathrm{~g}, n=23)$ when compared to hockey players $(35.0 \pm 1.7 \mathrm{~g}, n=128)$ and football players $(29.2 \pm 1.1 \mathrm{~g}, n=132)$; distributions of these impacts were also reported (Naunheim et al., 2000). Researchers also mounted triaxial accelerometers on custom-made headgear fitted to volunteer boxers in order to obtain human head kinematics and physiological effects. They found angular accelerations higher than $3500 \mathrm{rad} / \mathrm{s}^{2}$, exceeding proposed tolerance thresholds for volunteers; a maximum angular velocity of $48 \mathrm{rad} / \mathrm{s}$ and linear accelerations of 159 g were also reported (Chamouard et al., 1987; Pincemaille et al., 1989).

A device called the GForceTracker (GForceTracker Inc., Markham, ON) has been developed to measure head kinematics in conjunction with helmets, headbands, or goggle straps. One study implemented the GForceTracker in men's collegiate lacrosse to study head impact frequency and location; goalies had the highest magnitude of impact whereas face-off players had the lowest (Miyashita et al., 2016). Other technologies developed to integrate with headbands or skull caps include the Shockbox (Impakt Protective Inc., Kanata, ON), the 
Checklight (Reebok International, Ltd., Canton, MA and MC10 Inc., Cambridge, MA) and the SIM-P and SIM-G (Triax Technologies Inc., Norwalk, CT) (Cummiskey, 2015). The Shockbox was implemented in the helmets of 22 youth American football players to monitor head impacts (Wong et al., 2014), as well as in other, unpublished studies on American football and ice hockey (Patton, 2016). The Checklight has also been used to monitor head impacts in youth and high school football players (Harper et al., 2015), as well as in a soccer heading study (Wu et al., 2016).

Adhesive patches used to mount accelerometers to the skin, such as the xPatch (X2 Biosystems, Inc., Seattle, WA), have been employed to measure head response in soccer. Press \& Rowson (2017) used this sensor system to quantify head impact exposure in collegiate women's soccer over the course of a single season and found that $90 \%$ of the impacts per player were due to heading the ball. Other studies have similarly aimed to characterize head impact frequency and magnitude with the xPatch in collegiate and high school women's soccer (Lynall et al., 2016; McCuen et al., 2015; Stucker, 2015) and other sports (D. A. King et al., 2016; O'Sullivan \& Fife, 2017; Reynolds et al., 2016, 2017). The xPatch has also been employed in studies in order to evaluate the effectiveness of rule changes and education in youth football (Kerr et al., 2015; Swartz et al., 2015). Instrumented mouthguards are being developed as another means to study head biomechanics resulting from concussion (Camarillo et al., 2013; D.

A. King et al., 2015; Kuo et al., 2016; Siegmund et al., 2014; Wu et al., 2014), as are instrumented earplugs (Knox, 2004; Knox et al., 2009; Self et al., 2004).

While many of these technologies have been employed in research, it should be noted that most were developed for consumer use as opposed to research (Allison, 2015; Patton, 2016). As such, some of these devices have limited usefulness (Harper et al., 2015). Further, like the 
HIT system, all have questions of coupling and error in the kinematic measures as a result of the relative motion between the head and head gear, mandible motion or non-rigid skin coupling (Camarillo et al., 2013; Kuo et al., 2016; McCuen et al., 2015; Patton, 2016; Siegmund et al., 2015; Wu et al., 2016). Evaluation of these sensors has reported differences up to $150 \%$ in the raw data (Allison et al., 2015; Campbell et al., 2016). Reynolds et al. (2016) stated that reported values from head impact sensors measured in vivo should be taken with a grain of salt given that data from individual hits are noisy, a sentiment reiterated by Siegmund et al. (2015).

\subsubsection{Reconstructions of real-life impact events}

Head biomechanics research has also relied on reconstructions of real-life accidents to examine head injury patterns when wearable sensors cannot be used. A number of studies have been carried out to computationally investigate real-life accidents. Auer et al. (2001) studied 25

fatal pedestrian crashes to establish tolerance curves for acute subdural hematoma, subarachnoid hematoma, and contusions. Willinger et al. (2000) also studied real-life automotive crashes, this time involving motorcycles, by replicating motorcycle helmet damage through drop tests of instrumented helmets. The kinematics collected from the drop tests were used as input to FE models, which the authors then used to propose injury criteria. Multiple studies have similarly studied motorcycle, pedestrian, and car crashes via reconstruction (Dokko et al., 2003; Kang et al., 1997; Willinger \& Baumgartner, 2003). One in particular reconstructed nine head injuries from car crashes and noted that computer simulation tools were mature enough to obtain useful biomechanical information through reconstructions (Thomson et al., 2001). This sentiment of model utility was echoed by O'Riordain et al. (2003), who used a multibody dynamics program to reconstruct real-life falls resulting in head injury in order to compare simulation kinematic output with sustained injuries. 
A number of studies have numerically reconstructed falling accidents. Raul et al. (2006) used finite element modelling to distinguish between possible and impossible injury mechanisms for the observed lesions. Multibody and FE models of reconstructed falls have also been used to compare kinematics and strain results to findings by other researchers and published tolerances (Doorly \& Gilchrist, 2006, 2009; Doorly et al., 2005a, 2005b).

Sports injuries and impacts have also served as an input for computational and experimental reconstructions. Somers et al. (2011) computationally reconstructed race car impacts using an FE model to generate injury risk probabilities for concussion; a similar study was conducted by Begeman \& Melvin (2002). Multiple studies have reconstructed concussive head impacts from unhelmeted Australian football players using a multibody dynamics program to evaluate predictors of concussion severity (Fréchède \& McIntosh, 2007, 2009; McIntosh et al., 2014). Videos of unhelmeted Australian football players were studied to determine closing speed and other biomechanical characteristics of concussive head impacts, which served as an input to the multibody models (McIntosh et al., 2000).

Video analysis was also used in concussion cases from impacts in professional American football to determine the relative impact velocity (Pellman et al., 2003). These closing velocities were applied to Hybrid III ATDs to generate head kinematics associated with the concussive and non-concussive head impacts (Newman et al., 1999, 2005; Pellman et al., 2003). This set of head kinematics has since been applied to various FE models as researchers attempt to reconstruct the brain deformation associated with concussion (Ghajari et al., 2017; Giordano \& Kleiven, 2014; Kimpara \& Iwamoto, 2012; A. I. King et al., 2003; Kleiven, 2007; Newman et al., 1999; Viano et al., 2005; Willinger \& Baumgartner, 2003; Zhang et al., 2004). 
Post et al. (2017) similarly reconstructed youth head impacts in a laboratory using head contact velocities as their input for the reconstructions. The head kinematics were applied to FE models to investigate differences related to subjects that had a history of concussion versus those who had one concussion. Other studies have reconstructed head impact events in this way to investigate brain tissue response related to various forms of head injury (Kendall et al., 2012; Oeur et al., 2015; Post, 2013; Post et al., 2015). Head impacts have also been reconstructed using data from wearable sensors as the input. A pneumatic impactor matched HIT system kinematic data to match impact location and peak resultant linear and angular head accelerations measured on field with the reconstructed kinematic parameters (B. Rowson et al., 2017a, 2017b, 2017c). The reconstructed kinematics were then used in conjunction with linear regression to quantify correlations between various brain injury metrics and kinematic parameters (B. Rowson et al., 2017a, 2017b, 2017c).

While injury reconstructions provide a reasonable measure of the head biomechanics associated with an injurious impact, this manner of data collected can only provide an indirect measure of head response, causing uncertainty in the fidelity of kinematic data (Gilchrist \& Doorly, 2009). Further, questions surrounding the reconstruction process exist for both laboratory and computational reconstructions, such as the correctness of the data used for model inputs and initial conditions, fidelity of the human surrogate employed in the reconstruction, validity of the sensor data in an ATD, and even material properties of from a computational model (Gilchrist \& Doorly, 2009; Newman et al., 2005; O’Riordain et al., 2003).

\subsection{Data sources in this thesis}

For this thesis, there were several inclusion criteria that needed to be met in order for the data to be considered for use (Figure 2). Six DOF head kinematics, measured via sensors and not 
photo markers, were required. The kinematics were assessed to verify that peak values did not occur at the end of time history to allow sufficient data for calculating injury metrics and simulating brain deformations. For injurious impact data, only diffuse type brain injuries were included (e.g., concussion, DAI). For non-injurious head responses, symptoms needed to be reported in the literature. Non-injurious data sources were chosen based on input pulses so that some more vigorous or severe impacts (e.g. sled pulse $>10 \mathrm{~g}$ ) were included. This threshold was chosen based on minimum recording thresholds for wearable sensors, and previous studies have also defined a minimum threshold when studying injury as relatively low severity impacts are unlikely to cause MTBI (Duma et al, 2005; Funk et al., 2007; Reynolds et al., 2016). Evidence has shown that female athletes may be at greater risk for concussion than their male counterparts in comparable sports (Davis-Hayes et al., 2017; Dick 2009); and that concussive symptoms and neurocognitive function vary between male and female subjects (Broshek et al., 2005; Chiang Colvin et al., 2009). Literature has also shown that there are gender-specific risks and responses to various forms of brain injury, such as cerebral contusion and stroke (Barrett-Connor \& Bush, 1991; Roof et al., 1993; Cadet et al., 1994; Kannel \& Thom, 1994). There are several theories on the reason for this, including the vascular effects of estrogen (Mendelsohn \& Karas, 1994; Farhat et al., 1996) and differences in neuroanatomy (Chiang Colvin et al., 2009; De Courten-Myers, 1999); it is currently debated whether estrogen has a detrimental or neuroprotective effect in regards to concussion, but limited research has been published on estrogen's role in concussion risk (Covassin \& Elbin, 2011). Given the uncertainty between concussion tolerance in male and female subjects, only adult male subjects aged $18-45$ years were considered in order to minimize the effect of differences in anthropometry, age, and gender. Finally, the data had to readily accessible and available for use. 


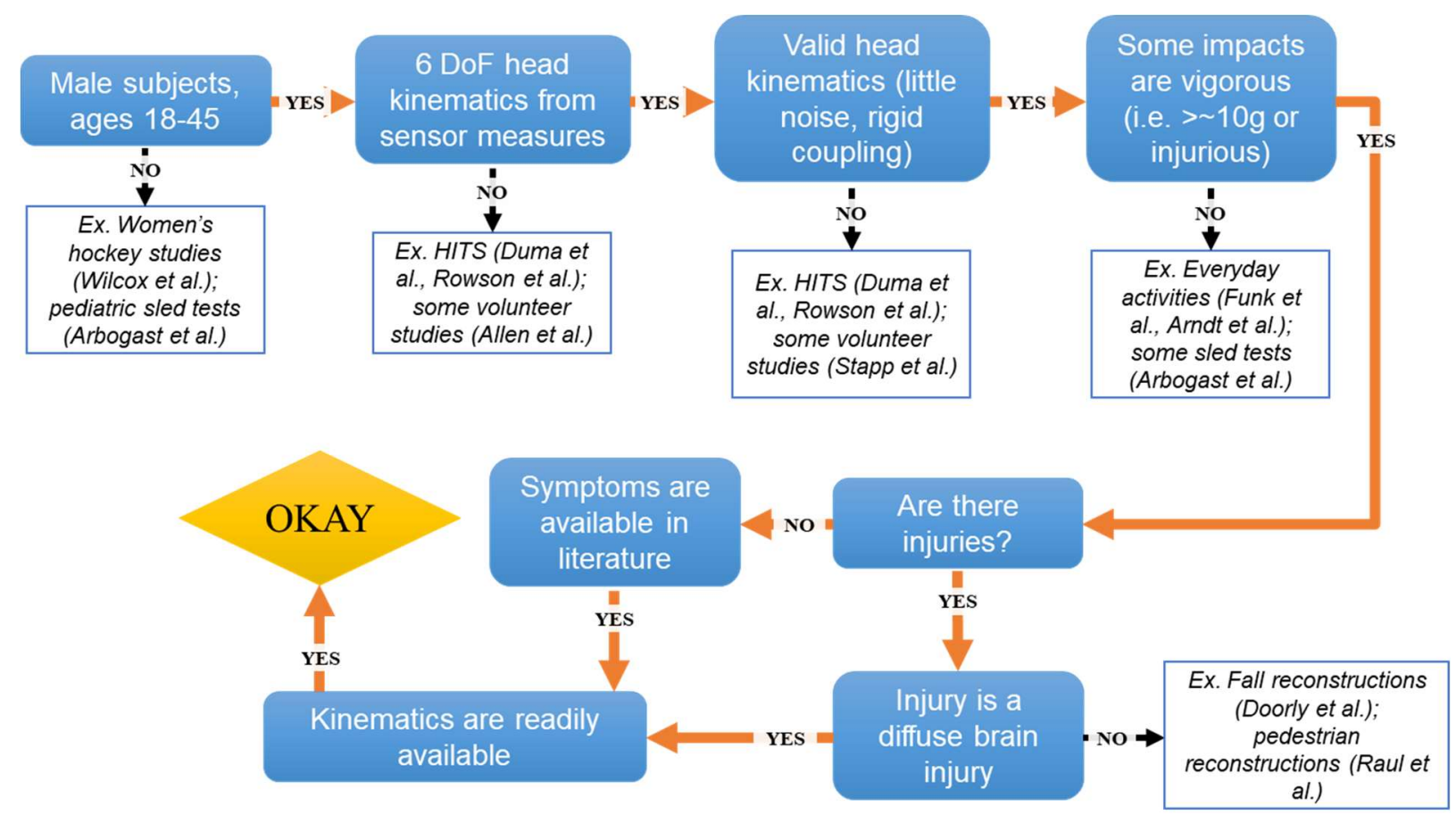

Figure 2. Flowchart of inclusion criteria to assist in the selection of data sources.

Of the data sources mentioned in section 2.2, three were chosen to use in this thesis using guidance from the inclusion criteria: data from of human research volunteer sled tests and professional football reconstructions. Datasets are comprised of healthy, male subjects, include complete measures of head kinematics, and have details on concussions diagnoses (if injured) and symptoms for the non-injurious dataset. Further details on the three data sources are outlined in the remainder of this section and a summary of the data can be found at the end of this chapter (Table 2).

\subsubsection{Human volunteer sled tests}

Three hundred thirty-five (335) individual, HRV sled tests were obtained from a larger database of sled tests performed by NBDL (Ewing et al., 1975, 1976, 1977, 1978, 1969; Ewing \& Thomas, 1972). Despite the wide variety of volunteer sled test data to choose from, the NBDL laboratory tests were the most extensive and included the highest severity sled pulses (16 g). 
Further, as outlined in the next chapter, the higher severity tests resulted in head kinematic values comparable to the football data sources described below.

A total of 22 individual volunteers were involved in these 335 sled tests. Volunteers were all adult males, aged 18 to 25 years, who served as sailors in the United States Navy. Prior to the start of testing, volunteers gave consent and underwent a qualification evaluation to ensure that all participants were healthy and had no prior deficiencies to put them at an increased risk of injury; extensive anthropometric measurements were also taken (Ewing \& Thomas, 1972).

Volunteers were restrained by a four-point belt in a seated posture on a sled buck and instrumented with angular rate sensors and accelerometers mounted at the mouth and over the posterior-superior aspect of the head (Ewing et al., 1969). The sled buck could be mounted in different orientations relative to the direction of the acceleration pulse; in this thesis, frontal $\left(0^{\circ}\right)$, oblique $\left(45^{\circ}\right)$, and pure lateral $\left(90^{\circ}\right)$ sled configurations were used (Figure 3). Sled accelerations varied in magnitude from 1 to $16 \mathrm{~g}$ and resulted in the inertial loading of the volunteer subject's head through the neck and torso. 

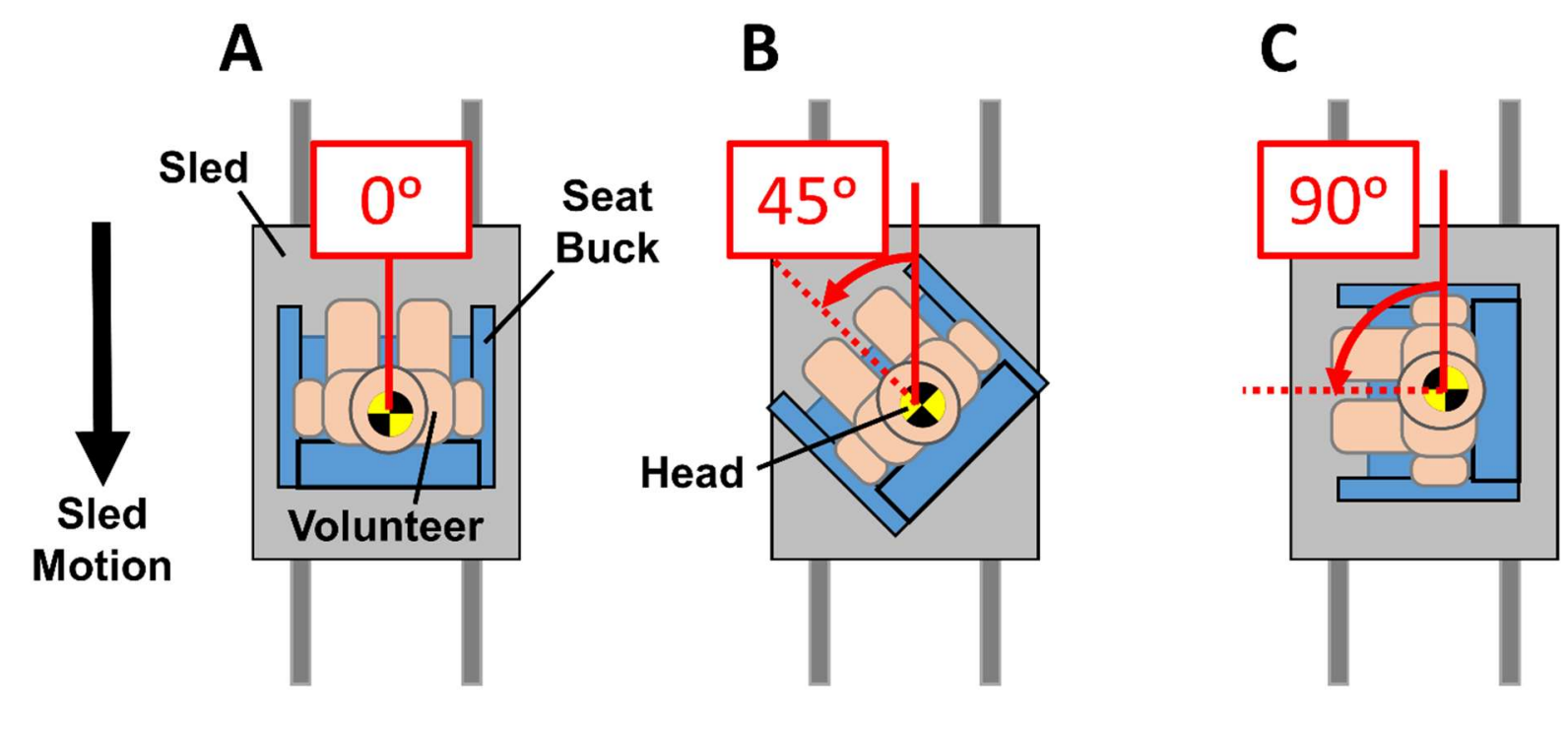

Figure 3. Volunteer sled test configurations for (A) frontal, (B) oblique, and (C) lateral.

Pre- and post-test medical examinations were conducted on each volunteer after every sled run to check for any developing ailments related to testing; generally, a follow up exam was performed 24 hours post-test (Ewing \& Thomas, 1972). A comprehensive examination was also completed at the end of the test series to ensure that no medical conditions existed that could be attributed to the sled runs. Medical reports were reviewed specifically looking for symptoms related to the current state of concussion and traumatic brain injury. Post-test clinical data from the HRVs revealed a history of headache occurring under the head anatomical mount, but was not considered neurologically significant (Ewing et al., 1969). Following the higher severity runs, some subjects had mild, musculoskeletal symptoms; however, these symptoms were transient and not considered clinically significant (Sanchez et al., 2017a).

\subsubsection{Professional football head impact reconstructions}

Video analysis and laboratory reconstructions were used to study concussive and nonconcussive head impacts in professional football (Newman et al., 1999, 2005; Pellman et al., 2003). Between 1996 and 2001, broadcast footage was collected from 21 football games and 
analyzed to estimate the location, direction, and speed for significant and concussive impacts. Initial impact conditions could be determined for 31 cases, of which 27 were helmet-to-helmet impacts and 4 were helmet-to-ground. Using the data from video analysis, a helmeted Hybrid III ATD head-neck was guided in freefall and struck a second ATD for helmet-to-helmet impacts or a simulated ground surface for helmet-to-ground impacts at the relative impact speed. Bungees helped position the moving and stationary ATDs, and rubber stoppers placed at the end of the track prevented the moving Hybrid III head-neck from falling off the track. In total, head kinematics for 58 players were reconstructed. Complete details on the video analysis and impact reconstruction methodology are available in literature (Newman et al., 1999, 2005; Pellman et al., 2003).

Concussive injury outcomes were gathered from team physicians who treated the players when injured using the definition introduced by the Committee on MTBI; this definition included similar diagnostic and symptomatic criteria to the 2012 Zurich Consensus definition of concussion (Pellman et al., 2004a, 2004b). Clinical and somatic symptoms were documented and fed into a database maintained to consider the epidemiology of MTBI (Pellman et al., 2004a). The cases were also independently verified by two team physicians using the clinical information, such as forms from the team physician and athletic trainer, and direct contact with medical staff for the injured player (Pellman et al., 2003). Symptoms for all concussions observed in professional football over the same time period as the reconstructions were reported by Pellman et al. (2004a). The most commonly observed symptoms included headaches, dizziness, and blurred visions, whereas the most common signs noted by physicians included problems with immediate recall, retrograde amnesia, and information-processing (Pellman et al., 2004a). In a subset of these data reconstructions consisting of 22 of the 25 concussed players, the 
most common symptoms included headache, memory problems, and dizziness (Viano et al., 2005); four of the 25 players also had loss of consciousness (Kleiven, 2007; Pellman et al., 2005).

\subsubsection{Reconstructions of collegiate football head impacts}

Fifty-five head impacts from were selected from a larger dataset of head impacts sustained by collegiate football players instrumented with the HIT system to be reconstructed in a laboratory (B. Rowson et al., 2017c). Players from 11 of these impacts resulted in concussion; the remaining 44 did not sustain a concussion. Impacts were reconstructed using a pneumatic linear impactor which struck a modified NOCSAE head form mounted on a Hybrid III $50^{\text {th }}$ percentile male neck instrumented with 3 linear accelerometers and 3 angular rate sensors. Impact locations reported by the HIT system were used as a starting point for each reconstruction, and impact speed and location were varied until peak resultant linear and angular accelerations fell within 5\% of the HIT system kinematic data (B. Rowson et al., 2017c).

Concussions in this dataset were defined as an alteration in the mental state resulting from a blow to the head, but not necessitating loss of consciousness (S. Rowson et al., 2012). Trained NCAA medical staff diagnosed all concussions using signs, symptoms, neurocognitive testing, and clinical judgment according to the guidelines set forth by the Second International Conference on Concussion in Sport in Prague. Symptoms included headache, nausea, vomiting, dizziness, fatigue, blurred vision, and difficulty concentrating (S. Rowson et al., 2012).

\subsection{Summary}

Researchers have seen the importance of human head kinematic data, especially in a field where the primary goal is to define human tolerance to injury. After outlining the availability of head kinematics data in the literature, inclusion criteria were determined to establish data sources 
to use for further analysis in this thesis. As described in section 2.3, three data sources were chosen; a detailed break-down of these data are provided below (Table 2). The remainder of this thesis focuses solely on analysis of this data and its application to injury risk functions.

Table 2. Summary of human head kinematic data sources used in this thesis.

\begin{tabular}{llll}
\hline \multicolumn{1}{c}{ Impact Type } & \multicolumn{1}{c}{ Data Source } & \multicolumn{1}{c}{ Test Details } & \multicolumn{1}{c}{$\begin{array}{c}\text { Sample Size } \\
\text { (Concussions) }\end{array}$} \\
\hline $\begin{array}{l}\text { Volunteer sled } \\
\text { tests }\end{array}$ & $\begin{array}{l}\text { Ewing \& Thomas } \\
(1972)\end{array}$ & $\begin{array}{l}\text { Volunteer sled acceleration } \\
\text { pulses up to } 16 \mathrm{~g} \text { at } 0^{\circ}, 45^{\circ},\end{array}$ & $335(0)$ \\
and $90^{\circ}$ & \\
$\begin{array}{l}\text { Collegiate } \\
\text { football } \\
\text { reconstructions }\end{array}$ & $\begin{array}{l}\text { Rowson et al. } \\
(2017 \mathrm{c})\end{array}$ & $\begin{array}{l}\text { Reconstructions with a } \\
\text { pneumatic impactor of on- } \\
\text { field impacts measured with } \\
\text { the HIT system }\end{array}$ & $55(11)$ \\
$\begin{array}{l}\text { Professional } \\
\text { football } \\
\text { reconstructions }\end{array}$ & $\begin{array}{l}\text { Pellman et al. } \\
(2003)\end{array}$ & $\begin{array}{l}\text { Helmet-to-helmet and helmet- } \\
\text { to-ground dummy } \\
\text { reconstructions of on field }\end{array}$ & \\
& & football impact &
\end{tabular}




\section{CHAPTER 3: ESTABLISHING A CONSISTENT DATASET}

\subsection{Introduction}

The first objective of this thesis was to establish a consistent dataset of concussive and non-concussive head impacts to use in evaluating head and brain injury risk functions. By calling the dataset consistent, this thesis means that only one injury diagnosis is considered (concussion). Further, the consistent definition is applied to the data, such that all data are processed in the same manner; this includes evaluating raw sensor measurements according to rigid body constraints, confirming head trajectories and/or kinematics via video review and other methods, identifying and eliminating secondary or artefactual impacts, and transforming the 6 DOF kinematics to the local head anatomic coordinate with an origin fixed at the head center-ofgravity (CG) and orientations defined according to SAE J211 (Figure 4; Comm, 2003). The dataset includes complete 6 DOF head kinematic time histories, which encompass a variety of magnitudes and durations. In this thesis, this dataset includes nearly $400(n=393)$ concussive and non-concussive head impacts taken from dummies and human volunteers; all data were obtained from previously published studies, as described in the previous chapter.

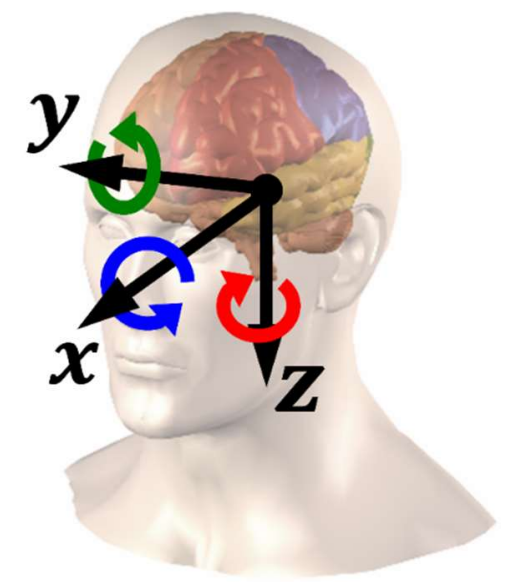

Figure 4. Local head anatomic coordinate system with orientations defined according to SAE J211. 


\subsection{Data preparation}

\subsubsection{Professional football reconstructions}

The professional football reconstructions were measured using a nine-accelerometer array package (NAAP or NAP) in the 3-2-2-2 configuration in order to calculate angular accelerations from rigid body constraints (DiMasi, 1995; Padgaonkar et al., 1975). The stability of the angular acceleration calculation using the NAP array stemmed from three extra measures of linear acceleration; however, it should be noted that these extra measures were also subject to rigid body constraints and as such, could be used to verify the consistency of each sensor measurement (Takhounts et al., 2009). Using rigid body constraints, Takhounts et al. (2009) derived a closed form, analytical solution for each accelerometer trace in the NAP array as a function of the array geometry and the other eight sensor measurements. If the analytical predictions matched the measured sensor traces, Takhounts et al. (2009) considered the NAP array consistent and would calculate rotational kinematics. Otherwise, if the analytical and measured traces did not match, the array was considered inconsistent and might have one or more sensor errors. One inconsistent accelerometer could be corrected by replacing its measured trace with the analytical solution, hereby preserving the required rigid body restraints for calculating 6 DOF kinematics (Takhounts et al., 2009).

The professional football reconstructions were evaluated for NAP consistency using the aforementioned method by Takhounts et al. (2009). When a NAP array was identified as inconsistent, each measured sensor trace was replaced with its analytical solution and new analytical solutions were calculated using the replaced sensor. The sum of squared errors (SSE) was calculated between the new analytical traces and the original; the combination that resulted in the smallest SSE value (i.e., the analytical solution that best matched the original traces) was 
identified and used to calculate 6 DOF head kinematics. Plots of consistent and inconsistent NAP arrays can be found in Appendix A.

Results of the sensor consistency check revealed that all the moving dummies and three of the stationary dummies had an inconsistent NAP array (Table 3). Minimum SSE was able to identify one channel corrections in the moving dummies: the Y-arm, X-direction sensor was identified as inconsistent in the first 12 reconstructions when organized chronologically and the Z-arm, Y direction accelerometer was identified in the remaining cases (Figure 5). The stationary dummy in the final three reconstructions identified the X-arm, Y sensor as erroneous. All corrected NAP arrays were reanalyzed for sensor consistency and found to be consistent with the exception of one reconstruction. Case 57, Player 2, had a $500 \mathrm{~g}$ spike in three NAP sensors which did not appear to be a physical response in the laboratory video. Given the three sensor error, the sensor traces in this reconstruction could not be corrected.

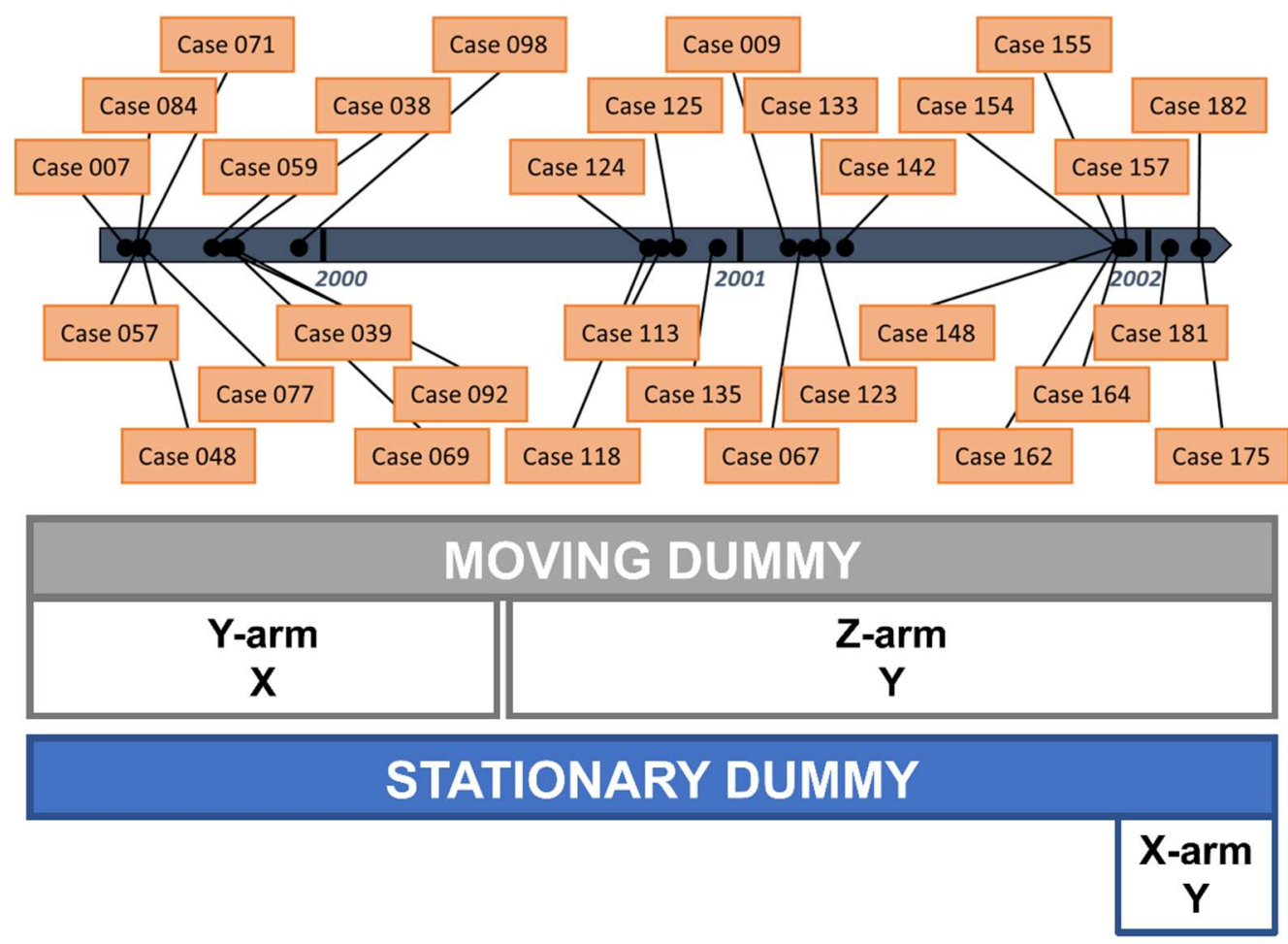

Figure 5. Chronological ordering of reconstructions showed that a common sensor was identified as inconsistent in subsets of the test series. 
Table 3. Inconsistent sensors in the NAP array and end times for each case. Blank rows under player 1 indicate helmet-to-ground impacts. Blank rows in the artefactual impact column indicate no secondary impact occurred.

\begin{tabular}{|c|c|c|c|c|}
\hline \multirow[b]{2}{*}{ Case } & \multicolumn{2}{|r|}{ Player 1} & \multicolumn{2}{|r|}{ Player 2} \\
\hline & End Time & Inconsistent Sensor & End Time & Inconsistent Sensor \\
\hline 57 & * & - & $x$ & $\dagger$ \\
\hline 84 & * & - & 40 & Y-arm X \\
\hline 48 & * & - & 44 & Y-arm X \\
\hline 77 & * & - & 36 & Y-arm X \\
\hline 71 & * & - & 66 & Y-arm X \\
\hline 59 & * & - & * & Y-arm X \\
\hline 39 & * & - & $x$ & Y-arm X \\
\hline 38 & * & - & 70 & Y-arm X \\
\hline 7 & * & - & 30 & Y-arm X \\
\hline 92 & * & - & 40 & Y-arm X \\
\hline 69 & * & - & 36 & Y-arm X \\
\hline 98 & * & - & 70 & Y-arm X \\
\hline 113 & * & - & 60 & Z-arm Y \\
\hline 124 & 42 & - & 42 & Z-arm Y \\
\hline 118 & 34 & - & 34 & Z-arm Y \\
\hline 125 & 48 & - & 48 & Z-arm Y \\
\hline 135 & 60 & - & 60 & Z-arm Y \\
\hline 9 & * & - & 52 & Z-arm Y \\
\hline 67 & & & $x$ & Z-arm Y \\
\hline 133 & & & $x$ & Z-arm Y \\
\hline 123 & & & $x$ & Z-arm Y \\
\hline 142 & & & * & Z-arm Y \\
\hline 148 & * & - & 76 & Z-arm Y \\
\hline 154 & * & - & 64 & Z-arm Y \\
\hline 162 & 100 & - & 60 & Z-arm Y \\
\hline 155 & * & - & 36 & Z-arm Y \\
\hline 164 & * & - & 36 & Z-arm Y \\
\hline 157 & * & - & 50 & Z-arm Y \\
\hline 175 & 40 & $X$-arm Y & 40 & Z-arm Y \\
\hline 182 & * & X-arm Y & 40 & Z-arm Y \\
\hline 181 & * & X-arm Y & 42 & Z-arm Y \\
\hline
\end{tabular}

*full time history can be used; NA: Not applicable; $\times$ secondary impact cannot be separated from reconstructed impact, reconstruction should not be used; †reconstruction may have multi-sensor error 
The corrected, calculated head kinematics were compared to the laboratory reconstruction videos to verify that the corrected kinematics matched the ATD motion. Euler angles were also calculated to match corrected head angles to the helmet motion seen in the laboratory reconstructions. In all cases, the corrected 6 DOF head kinematics were more consistent with the laboratory video, whereas the original head motions were not always representative of the motion seen. The laboratory videos were also reviewed to identify secondary impacts due to artefacts of the reconstruction. Artefactual events included impacts with the stopper at the end of the track or supporting bungees. Interactions were systematically identified and recorded for all reconstructions, as well as any possible impacts that occurred after the laboratory reconstruction videos ended. Videos and kinematics were reviewed to determine if the secondary impacts had enough temporal separation from the impact of interest. Sufficient temporal separation was defined such that the kinematics could develop (i.e., reach kinematic peaks and plateau) before the second impact occurred. End times based on the secondary impacts were then determined to dictate the region of applicability for each reconstruction.

Of the 58 reconstructed head kinematics, video analysis showed that 23 cases had no observed artefactual impacts and could be used in their entirety. On the other hand, five concussive reconstructions had secondary impacts that did not have sufficient temporal separation from the impact of interest. The head kinematics were therefore unable to fully develop and were not recommended for use in FE models. The remaining reconstructions ( $n=$ 30) had artefactual impacts sufficiently separated from the primary impact. These reconstructions impacted the end of the track $(n=14)$, a supporting bungee $(n=4)$, or the other ATD $(n=8)$, or kinematics indicated a potential impact after the laboratory video ended $(n=4)$. Head kinematics for these cases should be truncated between 30 and $100 \mathrm{~ms}$ (Table 3). 
The final, corrected dataset of head kinematics from the professional football reconstructions showed an overall increase in rotational head kinematics from the NAP corrections and the kinematic end times (Figure 6). There was no change in linear accelerations of any reconstruction because none of the CG linear accelerometers in the NAP array were identified as inconsistent; however, because five concussive reconstructions were eliminated from analysis, there was a slight decrease linear acceleration values as an entire distribution when comparing the corrected values to what was originally published (Figure 6).
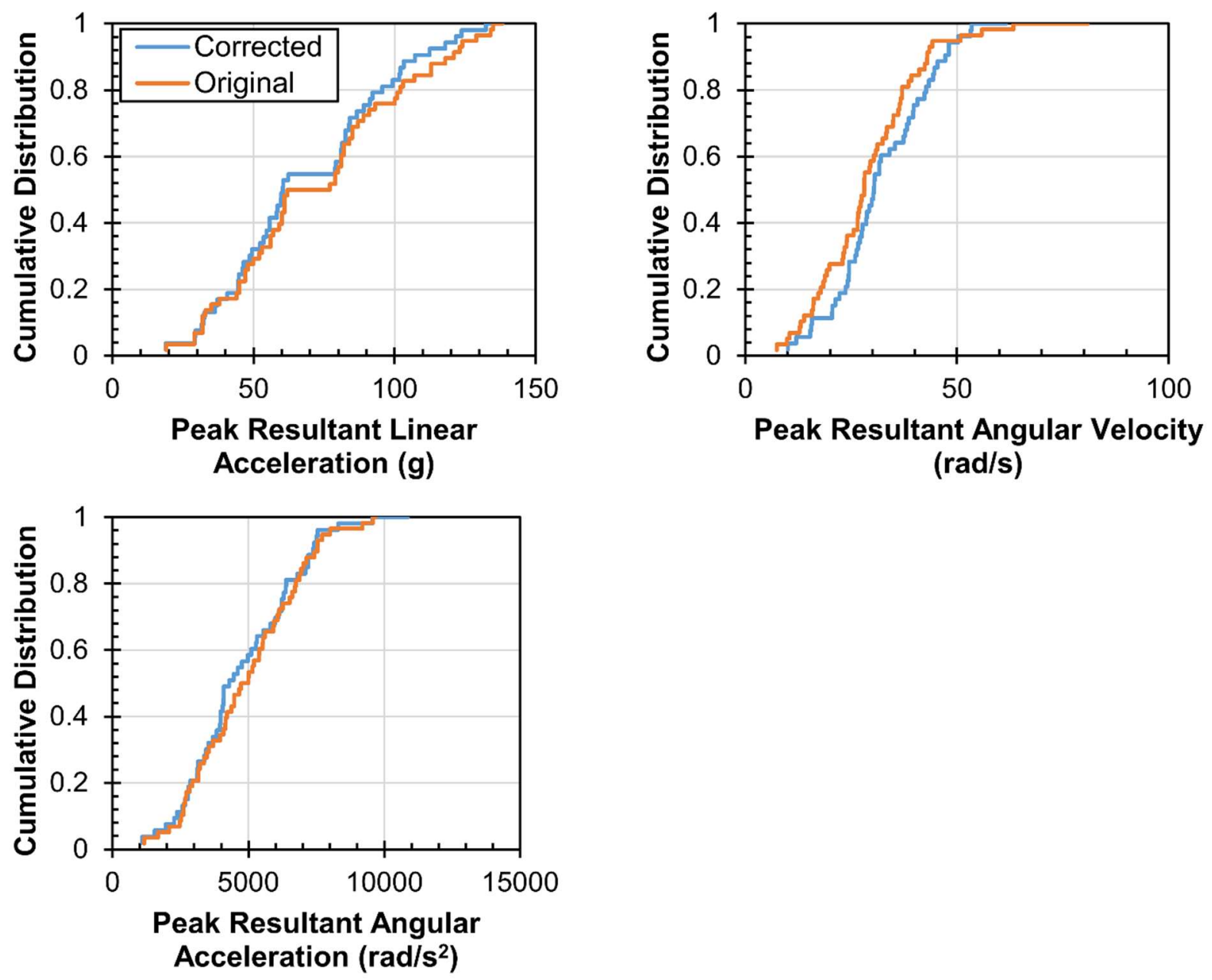

Figure 6. Change in distribution of the resultant head kinematics as a result of the corrections made to the professional football reconstruction data. 


\subsubsection{HRV sled tests}

Raw 6 DOF head kinematics from the HRV sled tests were measured in previous studies using a head-mounted sensor array and verified via high speed video analysis (Ewing \& Thomas, 1972; Thunnissen et al., 1995; Wismans et al., 1987). The head kinematics were previously processed using subject anthropometry to spatially transform the sensor data to a local head anatomic coordinate system near the head CG (Wismans et al., 1987). These data were obtained from the Netherlands Organization for Applied Scientific Research (TNO; $n=249$ ); head kinematics for an additional 86 sled tests were obtained from the publicly available biomechanics test database administered by the National Highway Traffic Safety Administration (NHTSA; ("NHTSA VSR" n.d.). The processed head kinematics included 3 linear accelerations, 3 angular accelerations, and 3 angular velocities of the head along the X, Y, and Z-axes. An additional spatial transformation was performed to define the data traces in a coordinate system with an origin fixed at the head $C G$ with the $+Z$ axis directed inferior (Figure 4 ). While the tests from the NHTSA database specified impact angles, the other 249 tests did not note the relative direction of the sled pulse with respect to the buck orientation. Trends in the head kinematics were observed, however, that suggested impact directions for these tests. Frontal sled pulses were characterized by high angular velocities about the $\mathrm{Y}$-axis of the head, with very little rotation about the other two anatomical axes. Similarly, tests in the lateral sled configuration had dominant rotations about the $\mathrm{X}$ - and Z-axes and little rotation about the $\mathrm{Y}$. Oblique tests showed significant angular velocity of similar magnitude in all three directions. Impact angles were confirmed by calculating head trajectories relative to the sled pulse (Figure 7). The dataset of HRV sled tests in this thesis consists of 132 frontal impacts, 97 lateral, and 106 oblique. 


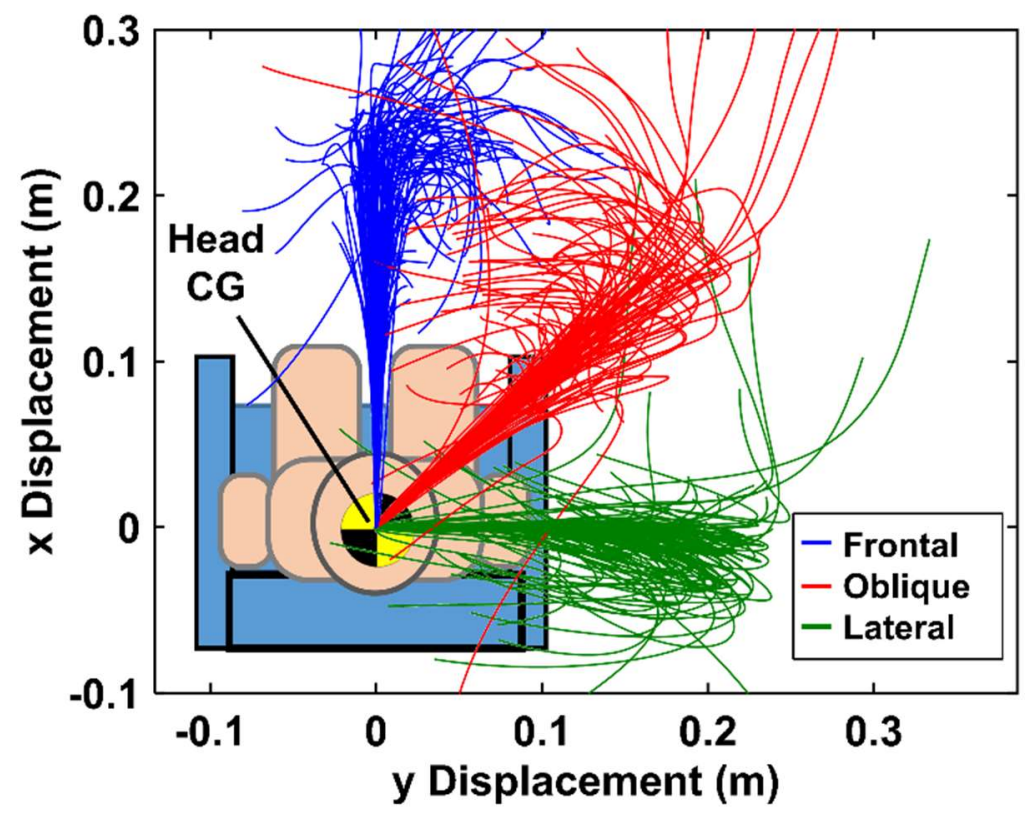

Figure 7. Linear displacement trajectories for each impact condition in the XY anatomical plane. Trajectories are plotted with respect to a coordinate system attached to the seat. The photo target indicates the location of the head $C G$.

\subsubsection{Collegiate football reconstructions}

Raw 5 DOF kinematics were previously measured during the 2004 to 2015 NCAA football season using the HIT system and used as an input for the collegiate football reconstructions such that the peak resultant linear and angular accelerations from the reconstruction fell within a 5\% margin of error from the original HIT system data. The kinematics from the reconstructions were then processed in a previous study (B. Rowson et al., 2017c, 2017a, 2017b). This processing included filtering the data to channel frequency class (CFC) 1000 for the linear accelerations and to CFC 155 for the angular rates using a four-pole, phase-less Butterworth low pass filter. Angular rate data were differentiated to find angular acceleration. For this thesis, processed data in the form of 3 linear accelerations and 3 angular accelerations were obtained and oriented with respect to the head anatomic coordinate system at 
the head CG. Angular velocities were calculated by integrating the angular accelerations using a backwards trapezoid method.

Distributions of the peak kinematic parameters were compared using the reconstructed data and the HIT system measures and saw that the distributions were closely matched, indicating agreement between the on-field and laboratory data (B. Rowson et al., 2017a, 2017b, 2017c). Further, impact locations were noted for the 11 concussive reconstructions. Head kinematics were assessed for agreement with the impact location and found to match.

\subsubsection{Data processing}

Head kinematics from all three data sources were processed using established techniques (Gabler et al., 2016a). Linear and angular accelerations were filtered to CFC 1000 (1650 Hz) using a zero-phase shift, digital 8 pole Butterworth filter, while angular velocities were filtered to CFC 60, or $100 \mathrm{~Hz}$ (NHTSA, 2015). Six degree-of-freedom processed head kinematics were used to calculate a variety of existing kinematics-based head and brain injury metrics and their respective injury probabilities (3.3) and as input for FE models (3.4).

\subsection{Kinematics-based injury risk metrics and injury risk functions}

Processed head kinematics were used to calculate several existing, kinematics-based head injury metrics and the associated probabilities of injury based on the injury risk functions. Metrics chosen for this thesis included a variety of translation and rotational head kinematic parameters based on several kinematic parameters, including linear acceleration $(\boldsymbol{a}(t))$, angular acceleration $(\boldsymbol{\alpha}(t))$, and angular velocity $(\boldsymbol{\omega}(t))$ time histories of the head. Each of the injury metrics also had an associated injury risk function published in the literature. All injury risk functions were developed using data involving either direct or indirect measures of human head kinematics or scaled animal data. Metric and risk function development is described below and is 
summarized at the end of this section (Table 4). Table 4 also contains values for the injury risk function coefficients.

\subsubsection{Peak resultant kinematics}

Injury risk curves predicting concussion were developed by Pellman et al. (2003) and Newman et al. (2000a) based on peak resultant linear head acceleration, $a_{\max }$, using reconstructed football head impacts. Pellman et al. developed two risk curves, one comprised of data only from the struck players and the second developed using data from both striking and struck players. Both injury risk functions by Pellman et al. were based on logistic regression analysis, taking the form of:

$$
P(x)=\frac{1}{1+e^{-g(x)}}
$$

Equation 1

where

$$
g(x)=\beta_{0}+\beta_{1} x
$$

Equation 2

is the basis function and $\beta_{i}$ are the fitted coefficients based on regression analysis. The curve by Newman et al. was digitized from a published plot to the same logistic form. Funk et al. (2007) also developed an injury risk curve for $a_{\max }$ from the same reconstruction data, but incorporated unbiased exposure data collected from collegiate football head impacts. Their injury risk function is based on a Weibull distribution:

$$
P(x)=1-e^{-\left(\frac{x-b}{\lambda}\right)^{k}}
$$

Equation 3

where $x$ is the maximum value of resultant linear acceleration and $\lambda, k$, and $b$ are the scale, shape and offset parameters determined from survival analysis.

Rowson et al. (2012) developed concussion-based risk curves for peak resultant angular acceleration, $\alpha_{\max }$, and maximum resultant angular velocity, $\omega_{\max }$, using data from collegiate 
football head impacts directly measured via the HIT system. The injury risk curves for these studies were based on logistic regression analysis (Equations 1 and 2).

\subsubsection{Head Injury Criterion (HIC)}

HIC, the current standard head injury metric for automotive safety and helmet design regulations, has been commonly used for evaluating head and brain injury potential. The mathematical formulation for HIC was derived by Versace, (1971):

$$
\mathrm{HIC}=\max _{\left(t_{1}, t_{2}\right)}\left\{\left(t_{2}-t_{1}\right)\left[\frac{1}{t_{2}-t_{1}} \int_{t_{1}}^{t_{2}}|\boldsymbol{a}(t)| d t\right]^{2.5}\right\} \quad \text { Equation } 4
$$

Resultant head acceleration, $|\boldsymbol{a}(t)|$, is expressed with units of $\mathrm{g}$. Times $t_{1}$ and $t_{2}$ have the unit of seconds and should be selected to maximize HIC over the full time history of the impact event (Versace, 1971); these values have also been constrained such that $t_{1}-t_{2} \leq 15 \mathrm{~ms}$ for current standards (Prasad \& Mertz, 1985). The injury risk curves used for HIC were developed from the expanded Prasad-Mertz curves using PMHS skull fracture data where the basis function, $\mathrm{g}(\mathrm{HIC})$, for Equation 1 was developed as follows:

$$
g(H I C)=\beta_{0}+\beta_{1} H I C^{-1}+\beta_{2} H I C
$$

Equation 5

\subsubsection{Generalized Acceleration Model for Brain Injury Threshold (GAMBIT)}

Newman (1986) saw the value of considering the combined effects of rotational and translational head motion when assessing for injury. Thus, he created GAMBIT as one of the first injury criterion to use both linear and angular head kinematics in the mathematical formulation:

$$
\operatorname{GAMBIT}=\max _{t}\left\{\left[\left(\frac{|\boldsymbol{a}(t)|}{a_{c r}}\right)^{2}+\left(\frac{|\boldsymbol{\alpha}(t)|}{\alpha_{c r}}\right)^{2}\right]^{1 / 2}\right\}
$$

Equation 6 
Critical values were defined as $a_{c r}=250 \mathrm{~g}$ and $\alpha_{c r}=25000 \mathrm{rad} / \mathrm{s}^{2}$ based on injury thresholds from literature. An injury risk curve for concussion using GAMBIT as the predictor variable was developed from 24 reconstructed football impacts using logistic regression (Equations 1 and 2; Newman et al., 2000b)

\subsubsection{Head Impact Power (HIP)}

Newman et al. (2000a) proposed a second injury metric which again combined translational and rotational head kinematics. HIP was developed based on a general expression for the rate of change of translational and rotational kinetic energy, also known as power. The coefficients were set equal to the mass $(m)$ and approximate mass moments of inertia $\left(I_{i i}\right)$ of a 50th percentile human head:

$$
\mathrm{HIP}=\max _{t}\left\{m \sum a_{i}(t) \int a_{i}(t) d t+\sum I_{i i} \alpha_{i}(t) \int \alpha_{i}(t) d t\right\} \quad \text { Equation } 7
$$

The principal moments of inertia of the head about the anatomical axes $\left(I_{i i}\right)$ were set to $0.016,0.024$, and $0.022 \mathrm{~N}-\mathrm{m}-\mathrm{s}^{2}$ for each direction, respectively, and $m$ is approximately equal to head mass $(4.5 \mathrm{~kg})$. Concussive and non-concussive football reconstructions were again used as the basis for a logistic regression (Equations 1 and 2) injury risk function for HIP (Newman et al., 2000a).

\subsubsection{Combined Probability of Concussion (CP)}

Rowson \& Duma (2013) introduced a new injury metric based on the values of maximum linear and angular acceleration of the head in order to predict the likelihood of sustaining a concussion for a given impact. The injury risk function was developed using logistic regression (Equation 1) based on instrumented collegiate football player data and used the following basis:

$$
g\left(a_{\max }, \alpha_{\max }\right)=\beta_{0}+\beta_{1} a_{\max }+\beta_{2} \alpha_{\max }+\beta_{3} a_{\max } \alpha_{\max } \quad \text { Equation } 8
$$




\subsubsection{Power Rotational Head Injury Criterion (PRHIC)}

Efforts have been made to modify HIC, which only uses translational kinematic measures, with rotational parameters. Kimpara et al. (2011) proposed PRHIC as brain injury criterion by replacing the linear acceleration term of HIC with the rotational component of HIP:

$$
\text { PRHIC }=\max _{\left(t_{1}, t_{2}\right)}\left\{\left(t_{2}-t_{1}\right)\left[\frac{1}{t_{2}-t_{1}} \int_{t_{1}}^{t_{2}} \mathrm{HIP}_{\mathrm{ang}} d t\right]^{2.5}\right\}
$$

where HIP ang is the angular component of HIP:

$$
\operatorname{HIP}_{\text {ang }}(\mathrm{t})=\sum I_{i i} \alpha_{i}(t) \int \alpha_{i}(t) d t
$$

Equation 10

Values for the mass moments of inertia $\left(I_{i i}\right)$ are reported above. A time duration constraint was set such that $t_{1}-t_{2} \leq 36 \mathrm{~ms}$ based on correlations with football head impact data. An injury risk function for PRHIC was developed with logistic regression using kinematic data from instrumented collegiate football impacts and professional football reconstructions (Equations 1 and 2; Kimpara \& Iwamoto, 2012).

\subsubsection{Rotational Injury Criterion (RIC)}

Kimpara \& Iwamoto (2012) proposed a second modification to HIC using rotational head kinematics. RIC was defined to have the same mathematical formulation as HIC, however, the linear acceleration term was replaced with angular acceleration.

$$
\mathrm{RIC}=\max _{\left(t_{1}, t_{2}\right)}\left\{\left(t_{2}-t_{1}\right)\left[\frac{1}{t_{2}-t_{1}} \int_{t_{1}}^{t_{2}}|\boldsymbol{\alpha}(t)| d t\right]^{2.5}\right\}
$$

Angular acceleration, $|\boldsymbol{\alpha}(t)|$, is expressed in $\mathrm{rad} / \mathrm{s}^{2}$ and a time constraint of $t_{1}-t_{2} \leq 36$ ms was again chosen from football impact data. An injury risk function developed for RIC was 
proposed by Kimpara \& Iwamoto (2012) based on logistic regression using the same dataset as the PRHIC risk curve (Equations 1 and 2).

\subsubsection{Brain Injury Criterion (BrIC)}

BrIC was developed to serve as a complement to HIC in the automotive industry in order to address HIC's inability to capture the rotationally induced brain injuries happening in car crashes (Takhounts et al., 2013). It was formulated from the maximum magnitudes of head angular velocity in three orthogonal components in order to incorporate the directional dependence seen in simulations of brain deformation.

$$
\operatorname{BrIC}=\sqrt{\left(\frac{\omega_{x}}{\omega_{x c r}}\right)^{2}+\left(\frac{\omega_{y}}{\omega_{y c r}}\right)^{2}+\left(\frac{\omega_{z}}{\omega_{z c r}}\right)^{2}} \quad \text { Equation } 12
$$

where $\omega_{i c r}$ are critical values as determined by FE simulation of head impacts from crash and pendulum tests $\left(\omega_{x c r}=66.25, \omega_{y c r}=56.45, \omega_{z c r}=42.87\right.$; Takhounts et al., 2013). Takhounts et al. (2013) developed injury risk functions for BrIC by correlating BrIC values with two strain metrics (MPS and CSDM) calculated from scaled animal data. As a result, BrIC injury risk probabilities may be calculated through either the MPS- or CSDM-based injury risk functions; this thesis uses both. Injury risk functions took the form of a Weibull distribution (Equation 3), where $x$ is BrIC. 
Table 4. Existing kinematics-based injury risk functions used in this thesis.

\begin{tabular}{|c|c|c|c|c|c|}
\hline $\begin{array}{l}\text { Metric } \\
\text { used } \\
\text { in IRF }\end{array}$ & $\begin{array}{c}\text { IRF } \\
\text { Reference }\end{array}$ & $\begin{array}{l}\text { Injury } \\
\text { Assessment } \\
\text { Type }\end{array}$ & $\begin{array}{l}\text { Data Source for IRF } \\
\text { Development }\end{array}$ & $\begin{array}{c}\text { IRF } \\
\text { Equations }\end{array}$ & $\begin{array}{c}\text { IRF } \\
\text { Coefficients }\end{array}$ \\
\hline$a_{\max }$ & $\begin{array}{l}\text { Newman et } \\
\text { al., (2000a) }\end{array}$ & Concussion & $\begin{array}{l}\text { Football impact } \\
\text { reconstructions }\end{array}$ & 1 and 2 & $\begin{array}{l}+\beta_{0}=-6.15 \\
\beta_{1}=7.91 \mathrm{E}-2\end{array}$ \\
\hline$a_{\max }$ & $\begin{array}{l}\text { Pellman et } \\
\text { al., (2003) }\end{array}$ & Concussion & $\begin{array}{l}\text { Football impact } \\
\text { reconstructions }\end{array}$ & 1 and 2 & $\begin{array}{l}\beta_{0}=-4.90 \\
\beta_{1}=6.06 \mathrm{E}-2\end{array}$ \\
\hline$a_{\max }$ & $\begin{array}{l}\text { Pellman et } \\
\text { al., (2003) }\end{array}$ & Concussion & $\begin{array}{l}\text { Football impact } \\
\text { reconstructions of } \\
\text { struck players only }\end{array}$ & 1 and 2 & $\begin{array}{l}\beta_{0}=-2.92 \\
\beta_{1}=5.59 \mathrm{E}-2\end{array}$ \\
\hline$a_{\max }$ & $\begin{array}{l}\text { Funk et al., } \\
\text { (2007) }\end{array}$ & Concussion & $\begin{array}{l}\text { Football impacts } \\
\text { measured with } \\
\text { wearable sensors and } \\
\text { reconstructions }\end{array}$ & 3 & $\begin{array}{l}b=0 \\
\lambda=397 \\
k=3.53\end{array}$ \\
\hline$\alpha_{\max }$ & $\begin{array}{l}\text { (S. Rowson } \\
\text { et al., } \\
\text { 2012) }\end{array}$ & Concussion & $\begin{array}{l}\text { Football impacts } \\
\text { measured with } \\
\text { wearable sensors }\end{array}$ & 1 and 2 & $\begin{array}{l}\beta_{0}=-12.5 \\
\beta_{1}=2.00 \mathrm{E}-3\end{array}$ \\
\hline$\omega_{\max }$ & $\begin{array}{l}\text { Rowson et } \\
\text { al., (2012) }\end{array}$ & Concussion & $\begin{array}{l}\text { Football impacts } \\
\text { measured with } \\
\text { wearable sensors }\end{array}$ & 1 and 2 & $\begin{array}{l}{ }^{*} \beta_{0}=-12.5 \\
\beta_{1}=4.42 E-1\end{array}$ \\
\hline $\mathrm{HIC}$ & $\begin{array}{r}\text { NHTSA, } \\
(1995)\end{array}$ & $\begin{array}{l}\text { Skull Fracture, } \\
\text { TBI }\end{array}$ & Human cadavers & 1 and 5 & $\begin{array}{l}\beta_{0}=-2.49 \\
\beta_{1}=-200 \\
\beta_{2}=4.83 E-3\end{array}$ \\
\hline GAMBIT & $\begin{array}{l}\text { Newman et } \\
\text { al., (2000b) }\end{array}$ & Concussion & $\begin{array}{l}\text { Football impact } \\
\text { reconstructions }\end{array}$ & 1 and 2 & $\begin{array}{l}+\beta_{0}=-6.78 \\
\beta_{1}=17.3\end{array}$ \\
\hline HIP & $\begin{array}{l}\text { Newman et } \\
\text { al., (2000a) }\end{array}$ & Concussion & $\begin{array}{l}\text { Football impact } \\
\text { reconstructions }\end{array}$ & 1 and 2 & $\begin{array}{l}+\beta_{0}=-4.68 \\
\beta_{1}=3.66 \mathrm{E}-4\end{array}$ \\
\hline $\mathrm{CP}$ & $\begin{array}{l}\text { Rowson \& } \\
\text { Duma, } \\
(2013)\end{array}$ & Concussion & $\begin{array}{l}\text { Football impacts } \\
\text { measured with } \\
\text { wearable sensors }\end{array}$ & 1 and 8 & $\begin{array}{l}\beta_{0}=-10.2 ; \\
\beta_{1}=4.33 \mathrm{E}-2 ; \\
\beta_{2}=8.73 \mathrm{E}-4 \\
\beta_{3}=-9.20 \mathrm{E}-7\end{array}$ \\
\hline RIC & $\begin{array}{l}\text { Kimpara \& } \\
\text { Iwamoto, } \\
(2012)\end{array}$ & Concussion & $\begin{array}{l}\text { Football impacts } \\
\text { measured with } \\
\text { wearable sensors and } \\
\text { reconstructions }\end{array}$ & 1 and 2 & $\begin{array}{l}+\beta_{0}=-7.04 \\
\beta_{1}=6.79 \mathrm{E}-7\end{array}$ \\
\hline
\end{tabular}




\begin{tabular}{|c|c|c|c|c|c|}
\hline PRHIC & $\begin{array}{l}\text { Kimpara \& } \\
\text { Iwamoto, } \\
(2012)\end{array}$ & Concussion & $\begin{array}{l}\text { Football impacts } \\
\text { measured with } \\
\text { wearable sensors and } \\
\text { reconstructions }\end{array}$ & 1 and 2 & $\begin{array}{l}\dagger \beta_{0}=-6.67 \\
\beta_{1}=7.58 E-6\end{array}$ \\
\hline $\begin{array}{l}\mathrm{BrIC} \\
\text { (MPS) }\end{array}$ & $\begin{array}{l}\text { Takhounts et } \\
\text { al., (2013) }\end{array}$ & DAI & $\begin{array}{l}\text { Scaled animal impact } \\
\text { data }\end{array}$ & 3 & $\begin{array}{l}b=0 \\
\lambda=0.602 \\
k=2.84\end{array}$ \\
\hline $\begin{array}{l}\text { BrIC } \\
\text { (CSDM) }\end{array}$ & $\begin{array}{l}\text { Takhounts et } \\
\text { al., (2013) }\end{array}$ & DAI & $\begin{array}{l}\text { Scaled animal impact } \\
\text { data }\end{array}$ & 3 & $\begin{array}{l}b=0.523 \\
\lambda=0.324 \\
k=1.8\end{array}$ \\
\hline
\end{tabular}

\footnotetext{
*reported to nominal injury risk values; $\uparrow$ data digitized from published plot
}

\subsection{Strain-based injury criteria and injury probability predictions}

Two FE models of the human head were used to obtain strain-based injury metrics (Figure 8). The Global Human Body Models Consortium-owed (GHBMC) 50th percentile male (M50) detailed seated occupant (v4.3) head and brain model was extra extracted from the whole body model and simulated independently by turning the dura into a rigid body (Mao et al., 2013). The second model, the Simulated Injury Monitor (SIMon v4.0), is a 50th percentile male human head model developed and distributed by NHTSA consisting of a brain and skull (Takhounts et al., 2008). Both models are comprised of the major brain structures, including the cerebrum, cerebellum, and brain stem, modelled as Kelvin-Maxwell viscoelastic materials. The skull was modeled as a rigid shell fixed to the head local anatomic coordinate system. The GHBMC brain model consists of over 120,000 elements and was validated against intracranial pressure data and relative brain-skull motion using PMHS data (Mao et al., 2013). The SIMon model has just under 45,000 elements and was similarly validated (Takhounts et al., 2008). Both models have been used in numerous computational brain injury. 
SIMon 4.0

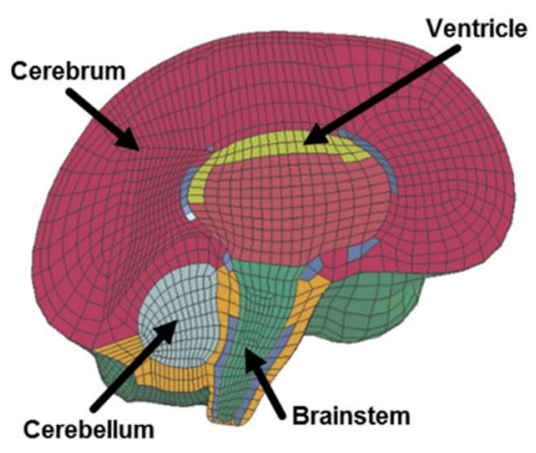

GHBMC 4.3

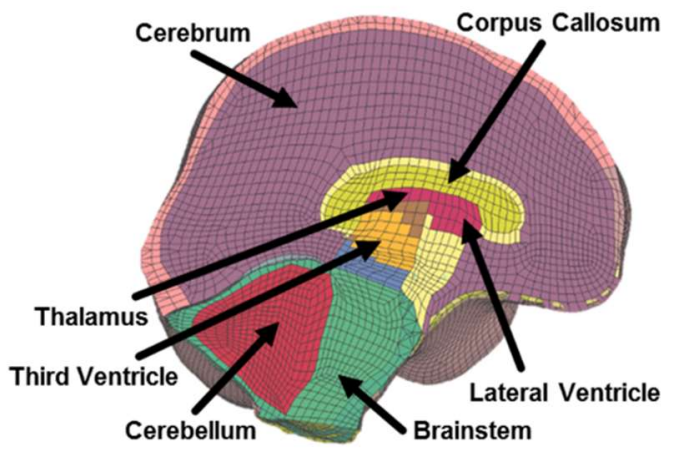

Figure 8. Mid sagittal plane view of the FE human head models used in this thesis.

The processed 6 DOF head kinematic time histories (3 linear accelerations and 3 angular velocities) were prescribed directly to the rigid skull of both FE models to calculate two injury criteria: MPS and CSDM. MPS is defined as the maximum strain occurring in all elements of the brain, while CSDM is the cumulative ratio of elements exceeding a set strain threshold (Figure 9). In this thesis, strain thresholds for CSDM of 0.15 and 0.25 were used based on previous work (Bandak, 1995; Sanchez et al., 2017b; Takhounts et al., 2003, 2013). A 95th percentile MPS value was also calculated to avoid potential numerical instabilities associated with the 100th percentile value of MPS (Panzer et al., 2012). Simulations were performed in LS-DYNA (v971 R7.1.1, double precision; LSTC, Livermore, CA).

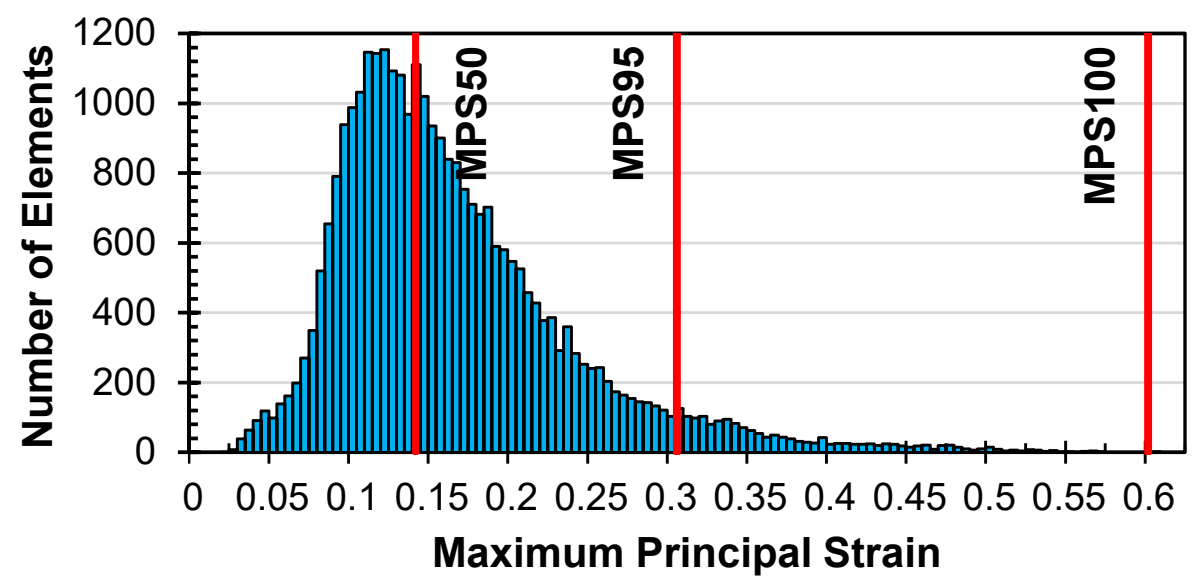

Figure 9. Distribution of MPS over all elements from the FE model and strain metrics calculated from the distribution. 
Injury risk functions for MPS100 and CSDM25 were previously developed using the SIMon FE model based on survival analysis of scaled animal data (Table 5; Takhounts et al., 2013). The injury risk functions took the form of a Weibull distribution (Equation 3). Injury risk functions have not been developed for the GHBMC strain metrics. As such, injury probabilities were only calculated for strain-based injury metrics from SIMon brain model. The GHBMC strain values of MPS95 and CSDM15 were chosen as baseline measures of brain deformation based on previous work (Gabler et al., 2016b; Takhounts et al., 2008; Sanchez et al., 2017b); they were used in later chapters to correlate with injury probability predictions from kinematicsbased and strain-based metrics. GHBMC strain values were also calculated to assess consistency between the two FE models.

Table 5. Strain-based injury risk functions used in this thesis.

\begin{tabular}{|c|c|c|c|c|c|}
\hline $\begin{array}{l}\text { Metric } \\
\text { used } \\
\text { in IRF }\end{array}$ & IRF Reference & $\begin{array}{l}\text { Injury } \\
\text { Assessment } \\
\text { Type }\end{array}$ & $\begin{array}{l}\text { Data Source for IRF } \\
\text { Development }\end{array}$ & $\begin{array}{l}\text { IRF } \\
\text { Equations }\end{array}$ & $\begin{array}{l}\text { IRF } \\
\text { Coefficients }\end{array}$ \\
\hline MPS & $\begin{array}{l}\text { Takhounts et } \\
\text { al., (2013) }\end{array}$ & $\mathrm{DAl}$ & $\begin{array}{l}\text { Scaled animal impact } \\
\text { data }\end{array}$ & 3 & $\begin{array}{l}b=0 \\
\lambda=0.505 \\
k=2.84\end{array}$ \\
\hline CSDM & $\begin{array}{l}\text { Takhounts et } \\
\text { al., (2013) }\end{array}$ & DAI & $\begin{array}{l}\text { Scaled animal impact } \\
\text { data }\end{array}$ & 3 & $\begin{array}{l}b=0 \\
\lambda=0.300 \\
k=1.8\end{array}$ \\
\hline
\end{tabular}

\subsection{Results}

\subsubsection{Head kinematics}

The HRVs were exposed to maximum resultant head linear accelerations up to $40 \mathrm{~g}$ (12.6 $\pm 6.8 \mathrm{~g})$ and peak resultant angular velocities and accelerations of $38 \mathrm{rad} / \mathrm{s}(18.4 \pm 7.7 \mathrm{rad} / \mathrm{s})$ and $2917 \mathrm{rad} / \mathrm{s}^{2}\left(743 \pm 490 \mathrm{rad} / \mathrm{s}^{2}\right)$, respectively, from the sled tests. Compared to the oblique and 
lateral tests, frontal sled configurations were generally able to achieve high sled acceleration pulses. However, lateral sled configurations typically resulted in higher rotational kinematics for the same sled pulse magnitude when compared with frontal and oblique impacts.

The collegiate football reconstructions had slightly more severe impacts, as well as incorporating some concussive outcomes. The non-concussed players saw an average linear acceleration of $60.1 \pm 21.2 \mathrm{~g}$; rotational kinematics measured $2757 \pm 1067 \mathrm{rad} / \mathrm{s}^{2}$ and $17.5 \pm 7.1$ $\mathrm{rad} / \mathrm{s}$ for angular acceleration and angular velocity, respectively. As expected, the concussive head impacts had higher values of maximum kinematics. These players experienced peak resultant linear acceleration of $114.5 \pm 37.8 \mathrm{~g}$, maximum resultant angular accelerations of 5430 $\pm 1956 \mathrm{rad} / \mathrm{s}^{2}$, and peak resultant angular velocities of $27.2 \pm 7.9 \mathrm{rad} / \mathrm{s}$.

In the professional football reconstructions, the non-concussed reconstructions had average linear and angular accelerations of $56.3 \pm 22.1 \mathrm{~g}$ and $3788 \pm 1315 \mathrm{rad} / \mathrm{s}^{2}$, respectively, while resultant angular velocity averaged around $27.7 \pm 8.8 \mathrm{rad} / \mathrm{s}$. The concussed cases generally achieved higher kinematic peaks; peak resultant linear acceleration had an average of $93.6 \pm 27.6$ $\mathrm{g}$, angular acceleration of $6776 \pm 1836 \mathrm{rad} / \mathrm{s}^{2}$, and angular velocity of $42.7 \pm 11.3 \mathrm{rad} / \mathrm{s}$.

Combining and comparing the datasets shows good overlap in the distributions of data. That is, the higher severity HRV data are similar and magnitude to the lower severity, nonconcussive football reconstruction kinematics, as are the magnitudes of the higher severity, nonconcussive football impacts and lower severity, concussive reconstructions (Figure 10). 

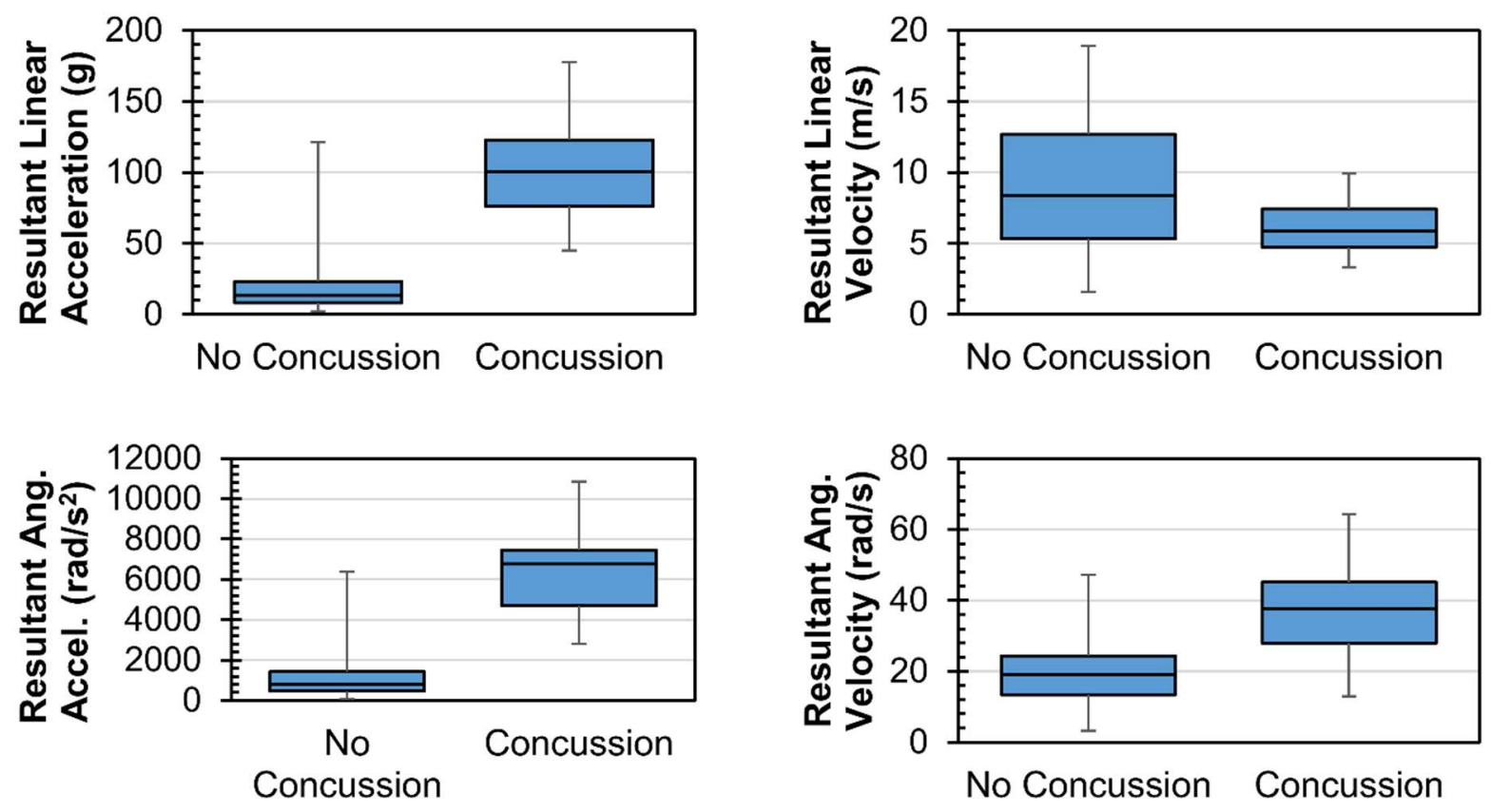

Figure 10. Distribution of maximum resultant kinematic parameters based on the 443 head impacts.

\subsubsection{Kinematic injury metrics and injury probability predictions}

Distributions for all injury metrics and injury probability predictions showed overlap between the concussive and non-concussive data (Table 6; Appendix B). The maximum resultant linear head acceleration of all non-concussive data (121.1 g) corresponded to a $2 \%$ (Funk), $97 \%$ (Newman), 92\% (Pellman - combined), and 98\% (Pellman - struck) risk of concussion, whereas the minimum injury probabilities from the concussive dataset indicated risks of $<1 \%, 7 \%, 10 \%$, and $40 \%$ for Funk, Newman, Pellman combined and Pellman struck, respectively. The peak angular velocity for non-concussed impact data (47.2 rad/s) indicated 100\% risk of concussion and peak angular acceleration $\left(6382 \mathrm{rad} / \mathrm{s}^{2}\right)$ showed a risk of $56 \%$; the concussive data had a minimum risk of concussion of $<1 \%$ for angular velocity (12.9 $\mathrm{rad} / \mathrm{s})$ angular acceleration (2795 $\left.\mathrm{rad} / \mathrm{s}^{2}\right)$. 
HIC values did not exceed 455 (32\% AIS 2+ injury) for the uninjured cases and had a minimum of 79 in the concussed data (1\%, AIS 2+). The lowest injury risks for PRHIC, RIC, $\mathrm{CP}$, and $\mathrm{HIC}$ in concussive cases were all less than $1 \%$, but had injury risk probabilities up to 92\% (PRHIC), 72\% (RIC), 24\% (CP), and 31\% (HIC) for non-concussive cases. HIP and GAMBIT had similarly low probabilities of concussion as the smallest value in concussive impact data ( $6 \%$ and $4 \%$, respectively); the maximum, non-concussed injury risk predictions were $96 \%$ for HIP and $85 \%$ for GAMBIT. The highest BrIC value in the non-concussive dataset was 0.85 , or a $93 \%$ risk of an AIS $2+$ injury using the MPS-based injury risk function and a $64 \%$ using the CSDM-based risk function. Concussive data had BrIC values as low as 0.24, corresponding to injury risk probabilities of approximately $7 \%$ according to the MPS-based injury risk function and less than $1 \%$ for the CSDM-based correlation.

Table 6. Peak non-concussive injury metrics and probabilities and minimum concussive injury metrics and risks.

\begin{tabular}{|c|c|c|c|c|}
\hline & \multicolumn{2}{|c|}{ Maximum Non-concussed Case } & \multicolumn{2}{|c|}{ Minimum Concussed Case } \\
\hline & Metric Value & $P(\operatorname{Inj})$ & Metric Value & $P(\operatorname{Inj})$ \\
\hline$a_{\text {max }}{ }^{*}$ & $121.1 \mathrm{~g}$ & $\begin{array}{c}2 \% ; 97 \% ; 92 \% \\
98 \%\end{array}$ & $45.1 \mathrm{~g}$ & $\begin{array}{c}<1 \% ; 7 \% ; 10 \% \\
40 \%\end{array}$ \\
\hline$\omega_{\max }$ & $47.2 \mathrm{rad} / \mathrm{s}$ & $100 \%$ & $12.9 \mathrm{rad} / \mathrm{s}$ & $<1 \%$ \\
\hline$\alpha_{\max }$ & $6382 \mathrm{rad} / \mathrm{s}^{2}$ & $56 \%$ & $2795 \mathrm{rad} / \mathrm{s}^{2}$ & $<1 \%$ \\
\hline HIC & 455 & $32 \%$ & 79 & $<1 \%$ \\
\hline PRHIC & 1.21E6 & $92 \%$ & 8960 & $<1 \%$ \\
\hline RIC & 1.18E7 & $72 \%$ & 1.63E6 & $<1 \%$ \\
\hline $\mathrm{CP}$ & - & $24 \%$ & - & $<1 \%$ \\
\hline HIP & 21403 & $96 \%$ & 5245 & $6 \%$ \\
\hline GAMBIT & 0.49 & $85 \%$ & 0.21 & $4 \%$ \\
\hline $\mathrm{BrIC} \neq$ & 0.85 & $93 \% ; 64 \%$ & 0.24 & $7 \% ;<1 \%$ \\
\hline MPS100 & 0.72 & $93 \%$ & 0.23 & $10 \%$ \\
\hline CSDM25 & 0.26 & $60 \%$ & 0.00 & $<1 \%$ \\
\hline
\end{tabular}

*Semicolons separate injury probabilities calculated from Funk, Newman, Pellman - combined, and Pellman - struck, respectively

¥Semicolons separate the MPS-based and CSDM-based probabilities of injury 


\subsubsection{Strain results and injury risks}

Strain-based metrics calculated through simulations using the SIMon FE model spanned a range of values. MPS100 varied between 0.10 and 0.72 for the non-concussive impacts, where an MPS100 of 0.72 corresponds to a 93\% risk of an AIS 2+ injury, and between 0.23 and 1.24 for concussive impacts. CSDM25 values peaked at 0.26 for non-concussive simulations, or approximately a $60 \%$ probability of AIS $2+$ injury. The concussed data had CSDM 25 values ranging up to 0.81 , spanning injury risk probabilities reaching $99.7 \%$. Distributions of the injury probabilities can be seen in Figure 11.

Compared to the SIMon simulations, the GHBMC model calculated similar results. MPS and CSDM values were generally lower in the GHBMC model, but overall correlations between the models were reasonable. MPS100 had the lowest correlation $\left(\mathrm{R}^{2}=0.83\right)$, while MPS95, CSDM15, and CSDM25 all showed better correlation $\left(\mathrm{R}^{2}>0.94\right)$. GHBMC strain-based metrics also spanned a range of values. MPS95 ranged from 0.01 to 0.37 in non-concussive simulations and between 0.15 and 0.64 in concussive ones; similarly, CSDM15 had values varied between 0.05 and 0.87 for concussive impacts and 0 through 0.57 from the non-concussive data (Figure 12).

\subsection{Summary}

Given that various injury metrics and risk functions have been proposed, it is not always clear which injury risk function performs best for specific loading conditions, much less across a variety of impact conditions. In this chapter, a dataset was established consisting both injurious and non-injurious head impacts with various loading conditions to use in evaluating brain injury risk functions. The data contained complete time histories of 6 DOF head kinematics and were assessed in terms of the validity of the sensor measurements and likeness to the impacts of the 

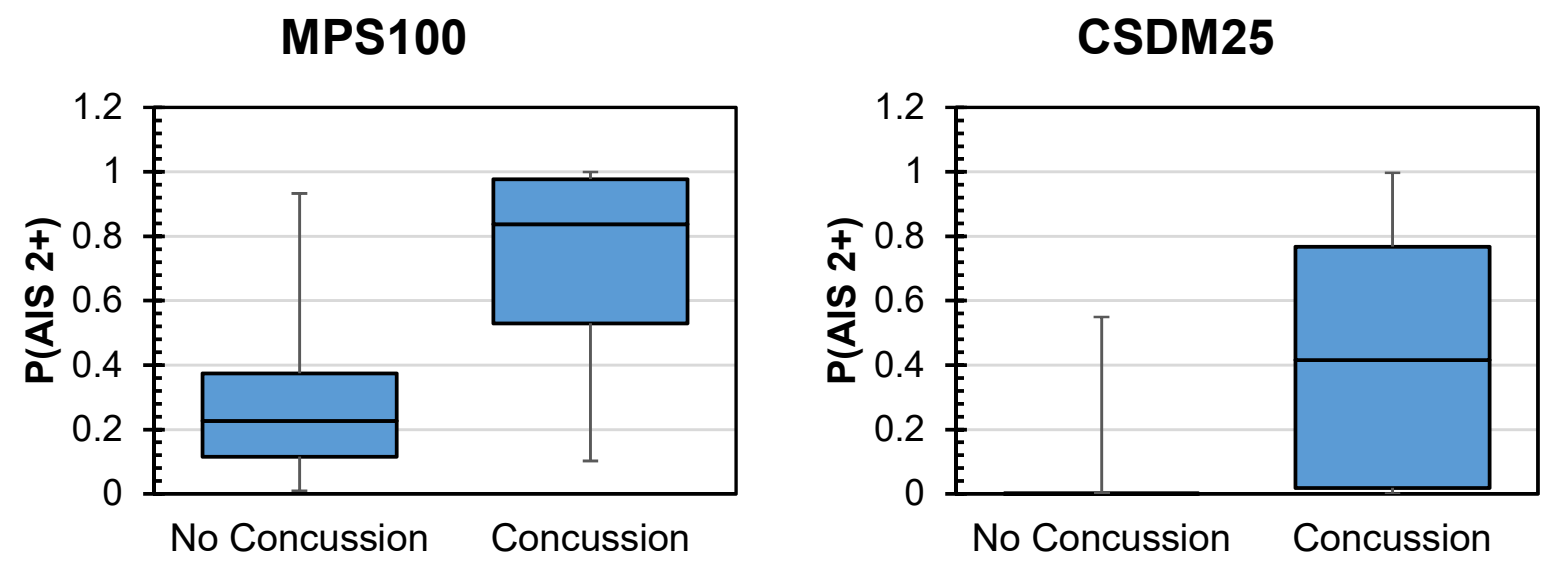

Figure 11. Distributions of the probabilities of injuries calculated from curves in Takhounts et al. (2013) using SIMon strain metrics as the predictor variable.
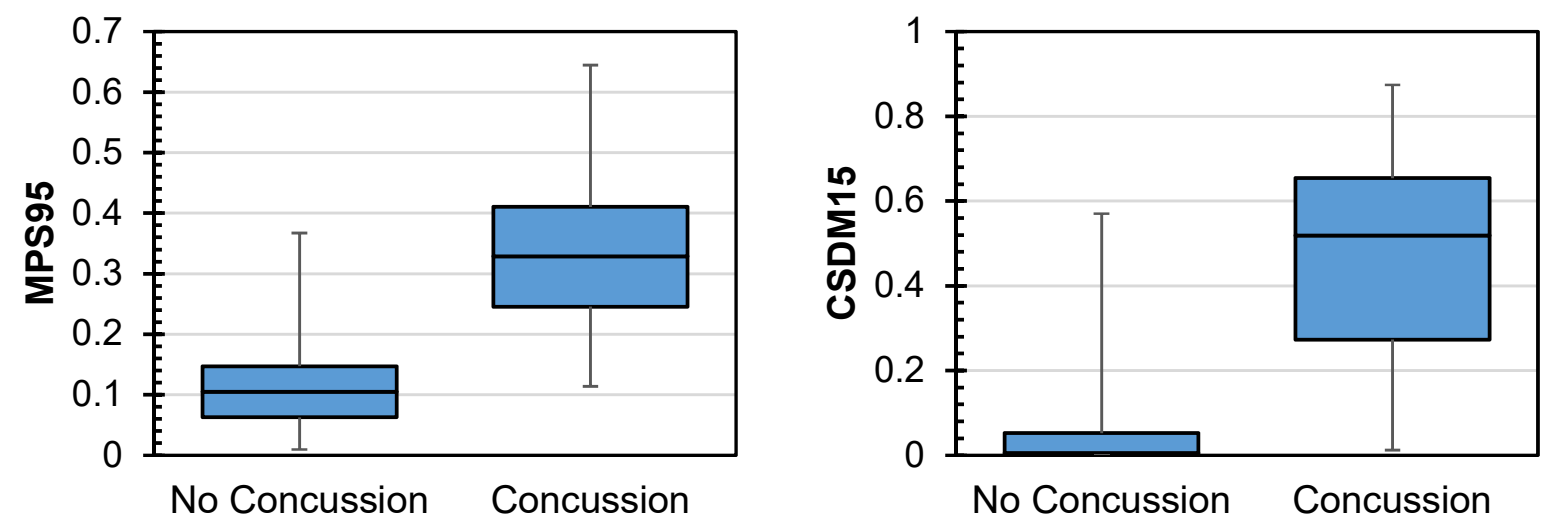

Figure 12. Distribution of strain metric values calculated via the GHBMC simulations.

sled tests or reconstruction impacts. Injury probabilities were calculated using risk functions for both kinematic and strain-based injury criteria. In the following section, these injury risk values are used in conjunction with their injury outcomes to assess the efficacy of the injury risk functions. 


\section{CHAPTER 4: EVALUATION OF CURRENT INJURY RISK FUNCTIONS}

\subsection{Introduction}

A fundamental goal of the field of injury biomechanics is to develop risk assessment models that can accurately assess a probability of injury based on one or more mechanical response variables. In chapter 2, a database of head impacts was established to be used to evaluate the risk functions outlined in chapter 3. This chapter employs various statistical analysis tools to evaluate the efficacy of the aforementioned injury risk functions. Studies have previously evaluated injury risk metrics and functions using correlations with strain (Gabler et al., 2016a; Kleiven, 2006) and through comparisons with field-based head injury rates. In this thesis, injury risk functions are similarly assessed via strain correlations. Their diagnostic capability is also evaluated using receiver operating characteristic (ROC) curves, and their ability to correctly predict injury outcomes over the whole dataset are assessed using expected injuries and calculations of likelihood estimates. The goal is to determine which metric can best distinguish between injurious and non-injurious impacts and how well each risk function predicts the observed injuries from the database.

\subsection{Correlations with strain metrics}

\subsubsection{Background}

Brain injuries from closed-head impacts cover a wide range of mild-to-severe types, including concussions and diffuse axonal injuries. Various mechanical variables have been proposed as brain injury predictors, such as intracranial pressure (Nahum et al., 1977; Zhao \& Ji, 2016). However, the primary belief is that these injuries are caused by tissue-level strains due to the inertial loading of the brain; numerous theoretical, experimental, and computational studies 
have supported this claim (Gennarelli et al., 1982; Holbourn, 1943). Given this link, researchers have strived to assess the correlations between head and brain injury metrics and strain metrics determined from FE models (Gabler et al., 2016a; Hernandez et al., 2015). The premise of these studies has been that injury metrics capable of predicting strain-based brain injuries should be well correlated with strain metrics. This idea also applies to injury risk functions; if injury risk functions can predict diffuse type brain injuries caused by brain deformation, they should have a relationship (or, be well correlated) with strain metrics.

\subsubsection{Results}

For each set of head kinematics, the injury probability estimated by each injury risk function was plotted with respect to the MPS95 and CSDM15 strain metrics calculated via GHBMC; correlations between the strain metrics and injury risk functions were determined using the entire dataset (Figure 13). The strongest correlates with MPS95 were MPS-based BrIC and the MPS100 injury risk function developed for SIMon with $\mathrm{R}^{2}$ values of 0.91 and 0.89 , respectively. Other injury risk functions that had good correlation with MPS95 include maximum angular velocity $\left(\mathrm{R}^{2}=0.84\right)$, HIP $\left(\mathrm{R}^{2}=0.77\right), \operatorname{CSDM} 25\left(\mathrm{R}^{2}=0.76\right)$, CSDM-based BrIC $\left(R^{2}=0.75\right)$ and a maximum linear acceleration curve $\left(\right.$ Pellman struck; $\left.\mathrm{R}^{2}=0.74\right)$. Funk et al.'s peak linear acceleration curve had the worst correlation with MPS95 $\left(\mathrm{R}^{2}=0.46\right)$, followed by CP $\left(\mathrm{R}^{2}=0.59\right)$. The injury risk functions developed for peak angular velocity, MPS95, CSDM25, MPS- and CSDM-based BrIC, HIP, all correlated well with CSDM15 $\left(\mathrm{R}^{2} \geq 0.80\right)$. Again, the worst correlations were with Funk et al.'s peak linear acceleration injury risk function $\left(\mathrm{R}^{2}=0.51\right)$ and the injury risk function developed for $\mathrm{CP}\left(\mathrm{R}^{2}=0.66\right)$. 


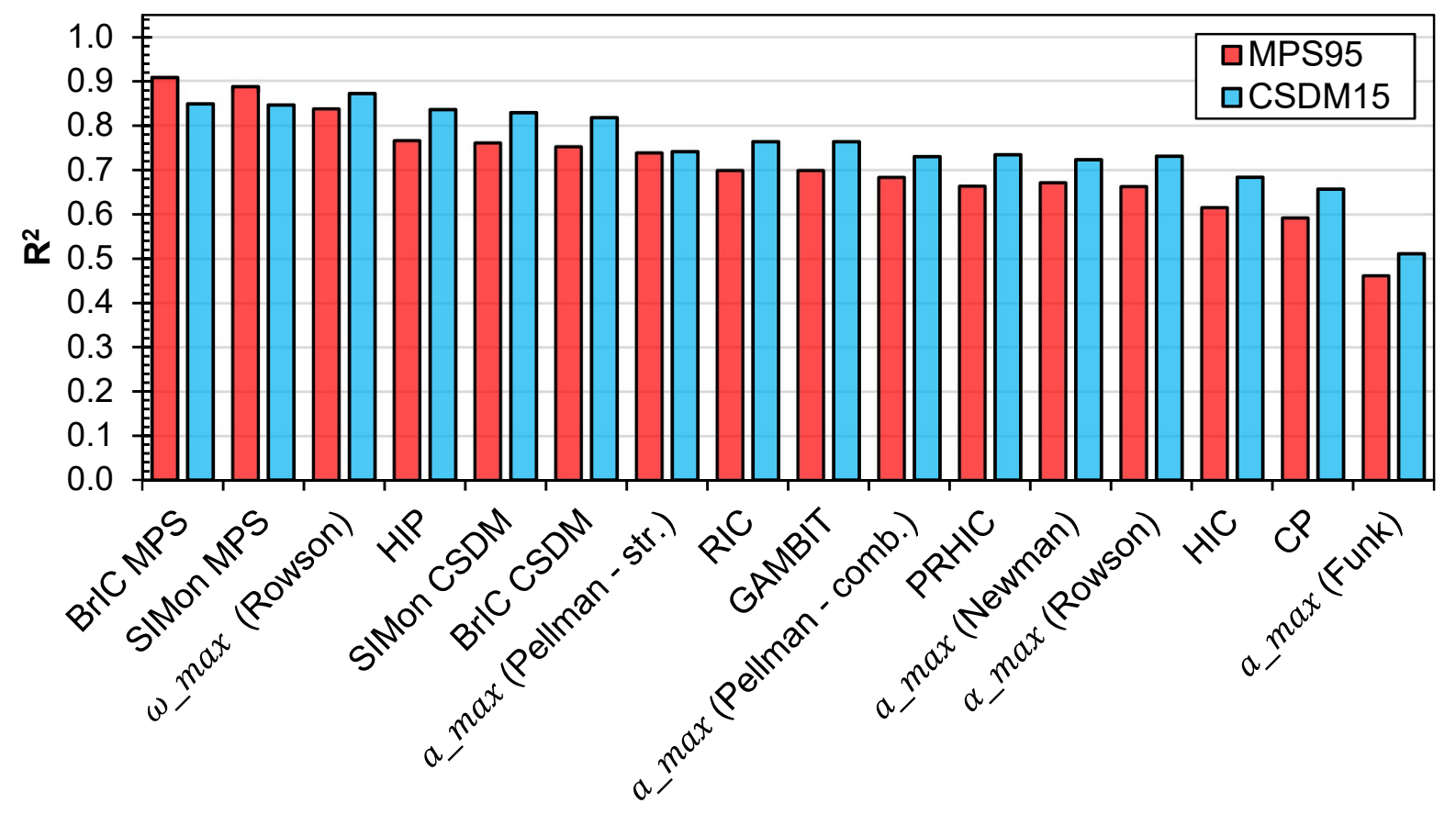

Figure 13. Overall correlation between strain metrics from GHBMC and injury risk probabilities from kinematic- and strain-based metrics.

\subsubsection{Discussion and Limitations}

When predicting brain strains as calculated with the GHBMC FE model, the strain-based injury probabilities unsurprisingly had some of the best correlations with strains. Although the injury risk functions were calculated from the SIMon model, strain metrics were well correlated between the two models (Figure 13). Overall correlation between the two models for MPS100 and CSDM25 were $\mathrm{R}^{2}=0.83$ and $\mathrm{R}^{2}=0.94$, respectively. Although MPS100 is used to calculate injury probabilities, this thesis used the $95^{\text {th }}$ percentile of MPS rather than the hundredth percentile value as the strain metric for correlations, as has been previously done (Gabler et al., 2016b; Takhounts et al., 2008). This made it so that simulation results were not driven by the response of a single element. Previous work has shown better model correlation using MPS95 (Gabler et al., 2016a); this is also the case for this dataset $\left(\mathrm{R}^{2}=0.93\right)$. 
Of all kinematic-based injury probabilities, the rotationally based risks had the strongest correlations with strain metrics; correlation values ranged from $\mathrm{R}^{2}=0.66$ to $\mathrm{R}^{2}=0.91$, where $\mathrm{R}^{2}$ values above 0.5 are considered to be moderately correlated while values above 0.7 are strong correlations (Rumsey, 2016). These good correlations are not surprising given previous work in literature of theoretical, experimental, and computational work to support this outcome (Gabler et al., 2016b; Holbourn, 1943; Ommaya \& Gennarelli, 1974; Takhounts et al., 2008, 2013). Injury risks calculated from translationally-based metrics were moderately correlated $\left(\mathrm{R}^{2}=0.46\right.$ to $\mathrm{R}^{2}=0.74$ ), while combination metrics (i.e., both linear and angular kinematic parameters) performed only slightly better than translational metrics.

While correlations were determined between injury risk probabilities and strain metrics, the correlations are likely more representative of the injury metric rather than the injury risk function. Injury risk functions are monotonically increasing and are generally one-to-one functions. In other words, every injury risk value corresponds to exactly one value of the injury metric or predictor variable, and as the predictor variable increases, so will the injury risk prediction. Previously published works have shown that rotational metrics to have better correlations with strain (Gabler et al., 2016a); this may support that these correlation values are more indicative of the injury metric themselves.

A limitation of the strain metric correlations is that they do not consider injury outcome. While this dataset showed that the concussive impacts generally had higher kinematic and strain values (Figure 10 and Figure 11), the actual injury outcomes were never considered in the correlations. Correlating the injury probabilities with strains showed that injury risk functions may be capable of predicting strain-based brain response, and while this thesis assumed that brain strains were indicative of injury, there could be concussive impacts with very little brain 
deformation and non-concussive impacts with large brain strains. Based on correlations alone, and not including observed injury outcomes, there is little way to differentiate between the injury risk metrics and the injury risk functions.

\subsection{Receiver operating characteristic curves}

\subsubsection{Background}

ROC curves are a means of assessing the performance of a metric for classifying data in two groups according to a binary response. Introduced by Egan (1975), the ROC curve illustrates the diagnostic capability of a predictor variable at various discrimination thresholds with respect to, in this thesis, a concussive or non-concussive outcome. An ROC curve plots the sensitivity of a dataset (i.e., true positive rate) versus 1-specificity (i.e., false positive rate) as the cut-off thresholds run through the range of all possible values. Different points on the curve correspond to the different thresholds used to determine which tests are considered a positive injury outcome (1)and which cases' injury risk values are considered uninjured (0; Rosner, 2015). The injury risk function which maximizes the number of true positives while minimizing the number of false positives on the ROC curve is identified as the curve closest to the upper left portion of the plot.

ROC curves are commonly compared by calculating the area under the curve (AUC; Faraggi \& Reiser, 2002). An AUC value of 1 represents a perfect predictor, meaning that there exists a threshold that perfectly divides the injured and uninjured data; this is drawn as a perfect right angle in the upper left corner of the plot. An AUC score of 0.5 serves as a practical lower limit for the AUC value, drawn as a line from the lower left corner $(0,0)$ to the upper right $(1,1)$ and indicative of random guessing; the AUC of each injury risk function can then be compared to random guessing to assess if the metric is a better indicator of concussion (Hanley \& McNeil, 
1982). Pairwise comparisons of the predictive capability of the injury risk functions can also be made to determine if any predictors are significantly different from others (Hanley \& McNeil, 1983).

ROC curves and their respective AUC values have been previously used to assess brain injury metrics. Greenwald et al. (2008) used AUC to determine which biomechanical measure was most sensitive to the prediction of concussion injury, defined as minimizing the false response rate. The study determined that a principal component score derived from HIC, SI, peak resultant linear acceleration, and peak resultant angular acceleration resulted in a lower false response rate when compared to each individual metric (Greenwald et al., 2008). ROC curves were also used to determine which threshold for CSDM was best used as a predictor of DAI (Takhounts et al., 2003); a threshold of 0.15 was chosen as the critical strain level and corresponded to experimentally determined strain values. Rowson \& Duma (2013) assessed the capabilities of linear and angular acceleration using AUC and in response, developed $\mathrm{CP}$ as a better indicator of concussion. However, while ROC curves and AUC have assessed brain injury metrics, they have yet to be used to assess the associated injury risk functions.

\subsubsection{Results}

ROC curves were drawn using the injury risk probabilities from each injury risk function (Figure 14). The AUC of each predictor was also computed for all ROC curves to compare the predictive capability of the different injury risk functions (Figure 14). As expected, all injury risk probabilities were statistically better than random guessing $(\mathrm{p}<0.01)$. The $\mathrm{CP}$ and angular acceleration injury risk functions by Rowson et al. (2012) and Rowson \& Duma (2013) were determined as the best predictors, with AUC values of 0.983 and 0.982 , respectively. However, most injury risk functions had AUC values above 0.90. The exceptions are rusk curves for 

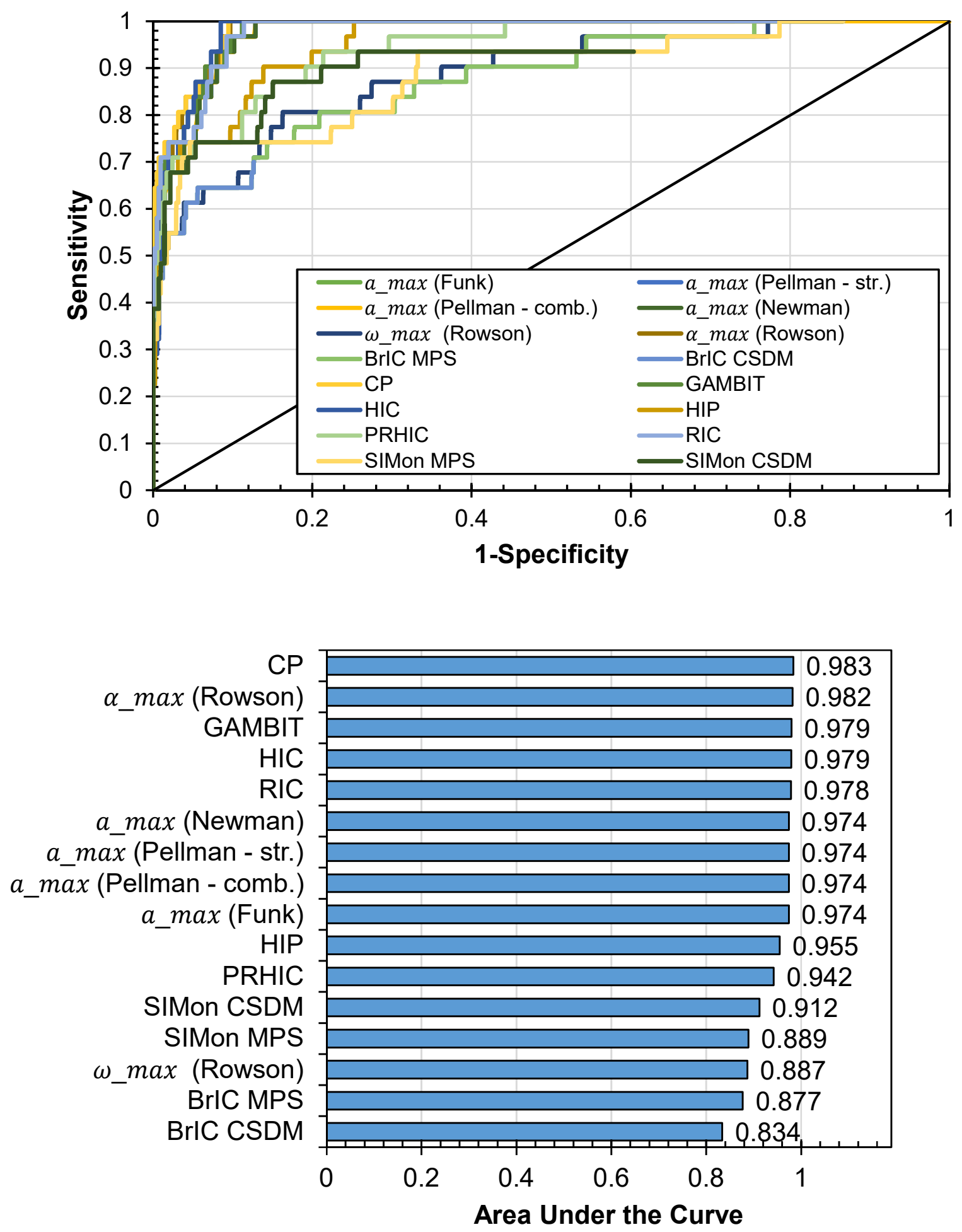

Figure 14. ROC curves (top) and their respective area under the curves (bottom) them of the injury risk probabilities estimated for various injury risk metrics 
MPS100 (0.889), peak angular velocity (0.887), and MPS- and CSDM-based BrIC (0.877 and

0.834, respectively). Pairwise comparisons of the AUCs using Hanley's method found statistically significant differences between the top 4 of the top AUC values (CP, GAMBIT, HIC, RIC) and the three lowest (BrIC MPS, BrIC CSDM, and peak angular velocity) at $\mathrm{p}<0.05$. There were also significant differences between peak angular velocity and peak angular acceleration AUC values, PRHIC with both BrIC MPS and BrIC CSDM, and BrIC CSDM with the SIMon CSDM AUC.

\subsubsection{Discussion and Limitations}

Using area under the ROC curve, results were quite different from the strain correlations; AUC values showed that injury risk values predicted from combination metrics ( $\mathrm{CP}, \mathrm{GAMBIT})$ performed best, while injury risk predictions based on angular velocity values performed worst (BrIC MPS, BrIC CSDM, peak angular velocity). Further, FE based injury risk probabilities had some of the lower AUC values, although these values were not significantly different from the higher AUCs. Compared to the strain correlations, the risk probabilities that had highest AUC values generally had the lowest correlation values. The reason for this could be that there was significant separation in the predictor variables. ROC curves are plots of how well the data can be separated by injurious outcomes; therefore, if the injury risk probabilities for the injurious cases are much higher than the non-injurious impacts, it would be easy to distinguish between the datasets and the AUC value would be high. Further, if many of the injury risks are near 0, this could set the injury risk function up to have good separation between concussive and nonconcussive impacts, but would worsen the correlations.

Similarly to the strain correlations, it is possible that the AUC values are indicative of the metric rather than the injury risk function. As previously mentioned, injury risk functions are 
monotonically increasing, therefore if, for example, the peak resultant angular acceleration values in the concussive impacts were much higher than the non-concussive cases, the injury risk values would also be much higher and would cause high AUC values. This also explains why the four linear acceleration risk functions have the same AUC. All use the same predictor variable (peak resultant linear acceleration) in their injury risk functions. While each point on each of these ROC curves may represent a different cutoff threshold, they have the same plot of true positive rate and false positive rate. The same could be expected from the two BrIC based injury risk curves. However, while both the CSDM- and MPS-based BrIC curves have the same input variable, the formulation of the CSDM-based curve assigns all BrIC values less than 0.523 a $0 \%$ risk of AIS 2+ injury, causing slightly different ROC values.

It is also important to note that some of the injury risk functions seem to have large separation between concussive and non-concussive injury risk probabilities, as seen in the boxplots in Appendix B. For example, CP has a median concussive probability of injury of 39\% and a $25^{\text {th }}$ percentile value of $9 \%$. On the other hand, the $75^{\text {th }}$ percentile injury risk value for the non-concussive impacts is a $0 \%$ injury probability. The large separation within the data likely explains the high AUC value. Several other risk functions have a similar concussive and nonconcussive distribution. Thus, these high AUC values are highly dependent on the underlying data being used in this assessment and could explain why some risk functions perform well in terms of ROC, but do not represent the mechanism of injury as illustrated by the strain correlations (section 4.2).

One property of ROC curves is their invariance under transformations. In other words, all the injury probabilities could be uniformly scaled down such that the maximum probability of injury was less than $10 \%$ and still maintain the shape of the ROC curve. This is because the 
shape of the ROC curve is not determined by the injury threshold, but rather by the ratio of true positives and false positives at all possible thresholds. Therefore, while an ROC curve can show the diagnostic ability and can determine thresholds for separating injury from non-injury, it may not be the best tool for evaluating the predictive capability of the injury risk functions as it does not assess the how well the injury risk probabilities correspond to injury outcome.

\subsection{Log-likelihood estimates}

\subsubsection{Background}

Unlike ROC curves and strain correlations, log-likelihood estimates assess a curve's ability to match a given outcome. The theory driving likelihood estimation is the belief that a good estimate of an unknown parameter would be a value that maximizes the probability, or likelihood, of getting a result similar to the observed data. For a fixed set of data and statistical model, the method of likelihood estimation chooses the model parameters that have the best chance of matching the observed outcomes. In terms of injury risk functions with parameters defined in literature and injurious outcomes, log-likelihood estimates can be used to assess how the predicted injury probabilities from each injury risk function match the injury outcomes. The log-likelihood estimates penalize injury risk values that are not indicative of the injury result; summing these values then ranks the injury risk functions. The function that maximizes the loglikelihood estimate, or as calculated below, minimizes the negative log-likelihood estimate, indicates a better predictor of injury.

Log-likelihood estimates were calculated by first performing the logit transformation on all calculated injury risk probabilities from each injury risk function. The logit function calculated the log-odds (i.e., the log of the probability that an event will happen divided by the

probability that the event will not happen) and was used to transform data in order to remove the 
boundary restrictions (i.e. $0<\mathrm{x}<1$ ). Negative log-likelihood estimates were then calculated from each of the transformed injury probabilities:

$$
\ln \left(1+e^{-\operatorname{logit}(\text { injury probability }) * \text { injury outcome }}\right)
$$

Equation 13

where

$$
\operatorname{logit}(x)=\ln \left(\frac{x}{1-x}\right)
$$

Equation 14

and

$$
\text { injury outcome }=\left\{\begin{aligned}
1, & \text { injury } \\
-1, & \text { non }- \text { injury }
\end{aligned}\right.
$$

In calculating the log-likelihood estimates, injurious cases with low injury risk probabilities and uninjured cases with high probabilities of injury risk were penalized more than uninjured cases that correctly estimated low injury risk values and injured impact data that correctly estimated a high injury probability (Figure 15). Log-likelihood estimates were summed over all cases for each injury risk function, and the risk curves were ranked by the sum of the log-likelihood values. It should be noted that the negative log-likelihood values were calculated, therefore lower values indicated better performance than higher values. 


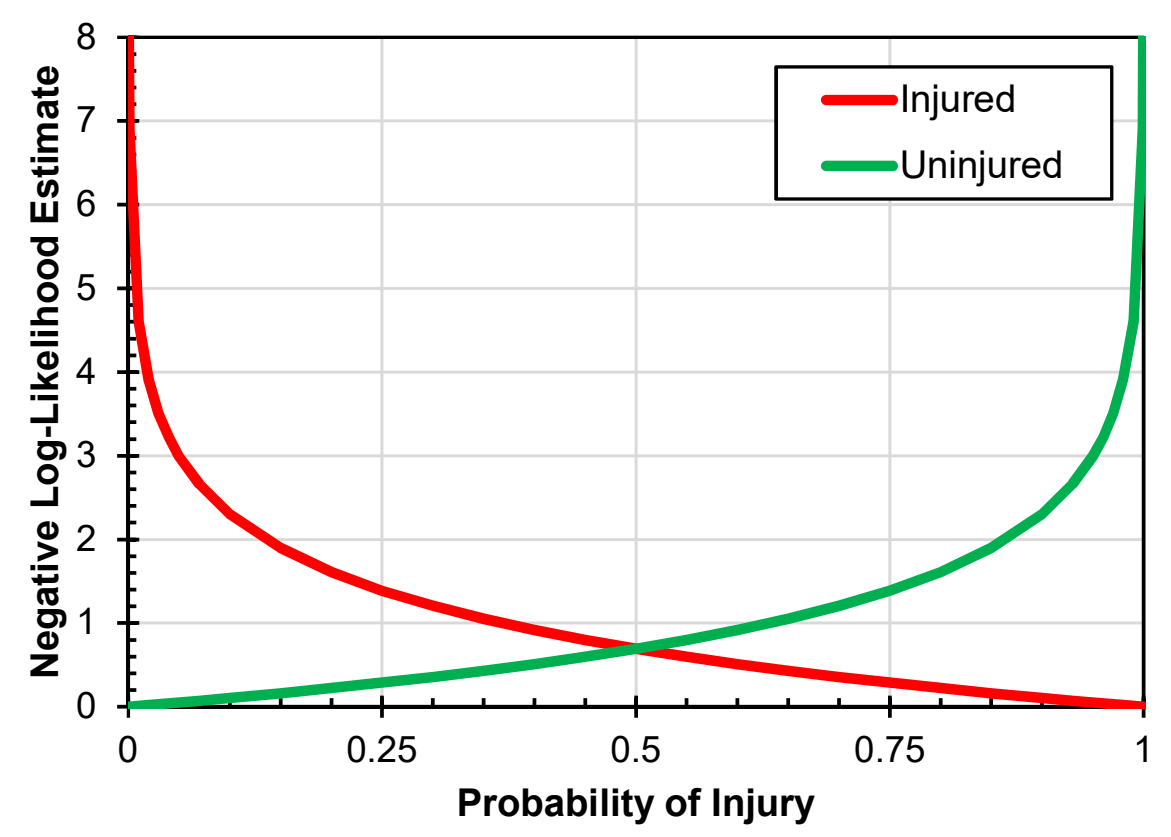

Figure 15. Plot of the negative log-likelihood estimates. Injurious cases (red line) that have a low probability of injury will have high values. The same is true with non-injurious cases (green line) that have a high risk of injury.

\subsubsection{Results}

Ranking the log-likelihood estimates relative to 31 concussions, the GAMBIT risk function and two peak resultant linear acceleration curves (Pellman combined and Newman) outperformed the other injury risk functions with estimates of 41.1, 52.7, and 52.8, respectively (Figure 16). Risk functions for CP, peak angular acceleration, RIC, HIP, HIC, PRHIC, and CSDM all had estimates under 100. The Pellman et al. struck and Funk et al. risk curves for peak linear acceleration had values of 123.2 and 156.0, respectively. While CSDM25 was ranked in the middle, the CSDM-based BrIC curve performed the worst, with a negative log-likelihood estimate of 209.2. The MPS-based BrIC (152.1) and MPS100 (161.9) risk functions ranked slightly higher than CSDM-based BrIC. Rowson et al.'s curve for peak resultant angular velocity had one of the largest negative log-likelihood estimates (177.7) despite being derived from the same data as the maximum resultant angular acceleration injury risk function. 


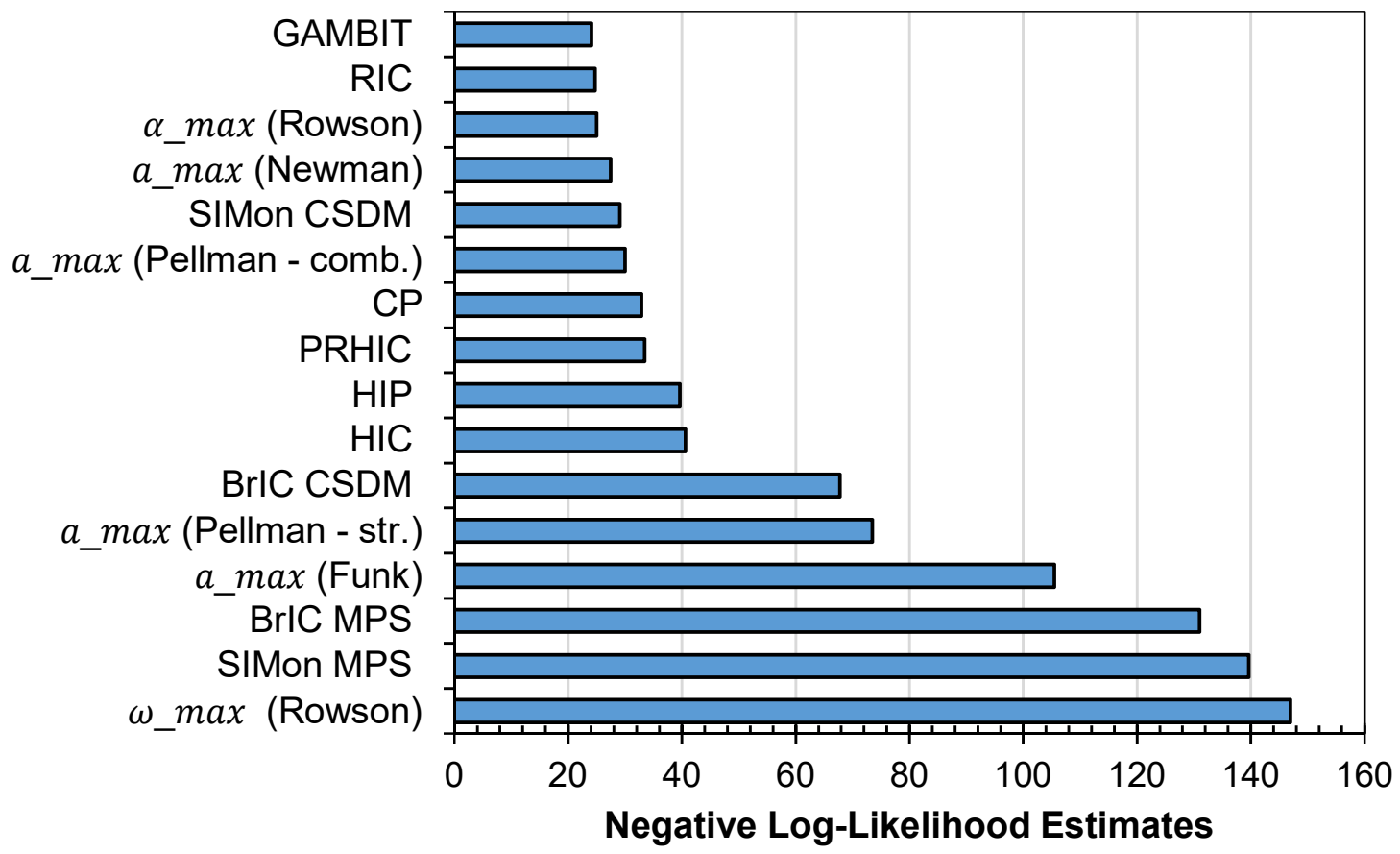

Figure 16. Negative log-likelihood estimates of all injury risk functions relative to 20 concussions.

\subsubsection{Discussion and Limitations}

Log-likelihood estimates are generally used to estimate the parameters of a statistical model given various outcomes by finding the parameter values that maximize the probability of the model making the correct observations given the parameters. In other words, maximum likelihood estimates help pick model parameter values so that they maximize the agreement of the observed data and calculated outcome. In this section, where the model parameters were previously determined in the literature, likelihood estimates were used to assess the parameter values given the injury observations from the database used in this thesis. The likelihood estimates then provided a ranking of how well each injury risk function predicts injuries based on the observed outcomes.

Using likelihood estimates, the risk curve for GAMBIT had the lowest value and was best able to predict the observed data outcomes. On the other hand, CSDM-based BrIC was the 
least capable of estimating outcomes based on the observed injuries. This result may be driven by eight of the concussive impacts, which all had BrIC values under 0.5 and an estimated $0 \%$ risk of AIS 2+ injury using the BrIC CSDM risk curve. Recall that injury probabilities that do not relate to their respective outcome are penalized (Figure 16); in this case the injury risk values $(0 \%)$ are the opposite of the injury outcomes (concussion) and greatly increase the likelihood estimate. Without these 8 impacts, the BrIC CSDM risk curve would have a likelihood estimate closer to 40 as opposed to 209 . At the same time, an injury risk function should never predict a $0 \%$ injury risk for a concussive injury outcome.

When calculating the log-likelihood estimates, the extreme injury probabilities are asymptotic when they are not indicative of injury outcomes. That is, when an injurious impact has a $0 \%$ probability of injury, the likelihood estimate approaches infinity. This is because the logit function is undefined at values of 0 and 1 ( $0 \%$ and $100 \%$ risk of injury). For all injury risk functions, there were cases of estimated $0 \%$ and $100 \%$ probability of injury. An offset of $10^{-9}$ was added to (or subtracted from) these injury risk values in order to calculate a finite value for plotting the likelihood estimates. However, an injury risk function should never predict a $0 \%$ probability of injury on a concussive case, nor should it predict a $100 \%$ probability of injury for a non-injurious case. Two injury risk functions, however, did predict a $0 \%$ injury risk for concussive cases: the BrIC CSDM and the SIMon CSDM risk curves.

A limitation of log-likelihood estimates is that there is no threshold that defines a good fit or a bad fit. Likelihood estimates are used to determine parameters for the best fit of a model, where the best fit maximizes the likelihood of correctly estimating the observations and therefore has the lowest likelihood estimate. Thus, likelihood estimates are only capable of ranking the 
injury risk functions in terms of ability to predict the injury functions. It cannot be used by itself to determine good or poor model fit.

\subsection{Expected injuries}

\subsubsection{Background}

A second method of assessing the ability of an injury risk function to correctly predict injury outcomes over a dataset is through expected injuries or the expected value. In statistics, the expected value of a random variable is the average result of an experiment after many repetitions, or what should be anticipated as the result of many trials. For example, the expected value of rolling a die is 3.5. In regression analysis with binary outcomes, the expected value of the regression probabilities should be close to the number of observed outcomes, where large the differences between observed values and expected values indicate poor the fit of the model to the data. The number of expected injuries was calculated using this database to compare the injury risk function estimates to the number of observed injuries. Injury probabilities were summed over all the tests to obtain the expected number of injuries for each of the 16 injury risk assessment tools. The accuracy of each injury risk function was evaluated by comparing the expected number of injuries relative to the injury diagnoses in this cohort $(n=31)$.

\subsubsection{Results}

The injury risk function developed for GAMBIT estimated 35 expected injuries, the closest prediction of expected injuries out of all injury risk functions when compared with the number of observed injuries ( $n=31$; Figure 17). Injury risk functions developed for RIC, maximum resultant angular acceleration, PRHIC, CSDM25, and BrIC CSDM estimated 18 to 20 expected injuries, compared to 31 observed injuries. While the CSDM-based BrIC and CSDM25 
risk functions slightly underestimated the number of injuries, their counterparts of MPS-based BrIC and MPS100 overestimated the expected injuries with values of 121 and 131, respectively. These were the highest seen for all risk functions. The peak linear acceleration curves showed mixed results. The Pellman struck (105), Pellman combined (47), and Newman (45) curves overestimated the count of observed injuries, while the curve developed by Funk calculated less than 1 expected injury in the entire dataset, severely underestimating the number of injuries. The maximum angular velocity curve estimated 87 expected injuries, compared to the 20 predicted by the peak angular acceleration risk function developed from the same dataset. HIP also overestimated the number of injuries (48), while CP and HIC underestimated them (14 and 10, respectively).

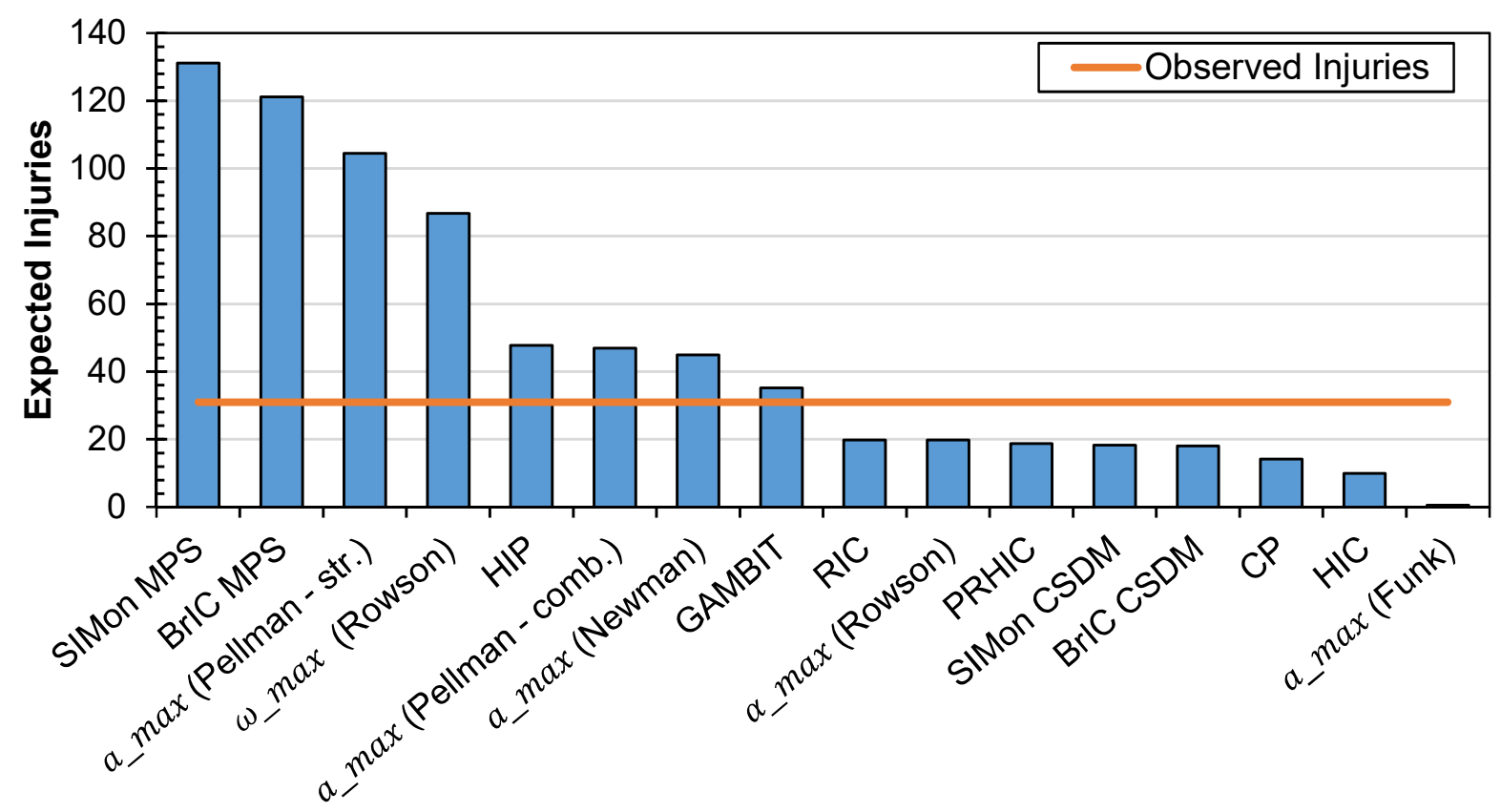

Figure 17. Expected number of AIS 2+ and concussive injuries predicted using each of the injury risk functions calculated using the full dataset $(n=443)$. The full dataset had 31 observed concussions.

Sanchez et al. (2017), in a similar analysis, noted that an assumption of the volunteer sled tests is that each test is independent, when in fact, all 335 sled tests were conducted using 22 subjects. As such, it would be surprising if a volunteer was not injured on the most severe sled 
pulse but was injured at a lesser exposure. While sled test data were available for all $22 \mathrm{HRVs}$, subject-specific identifiers were only available for the 249 sled tests obtained from TNO (16 subjects). For each of these volunteers, the most severe sled run, determined by highest injury risk, was chosen for each of the injury risk functions examined. In some cases, individuals achieved their highest injury risks in different sled tests for different risk functions. For each risk function, the injury probability of the most severe runs for each subject was collected and summed to obtain the number of expected injuries from the smaller cohort of data. Recalculating expected injuries using only the most severe sled runs found that trends were similar to before (Figure 18). GAMBIT again had the nearest estimate of expected injuries to observed (34 expected compared with 31 observed). BrIC-based MPS, MPS100, and the Pellman struck linear acceleration curves resulted in the largest expected injuries (54, 58, and 73 expected injuries), while Funk's linear acceleration curve again estimated less than 1 injury.

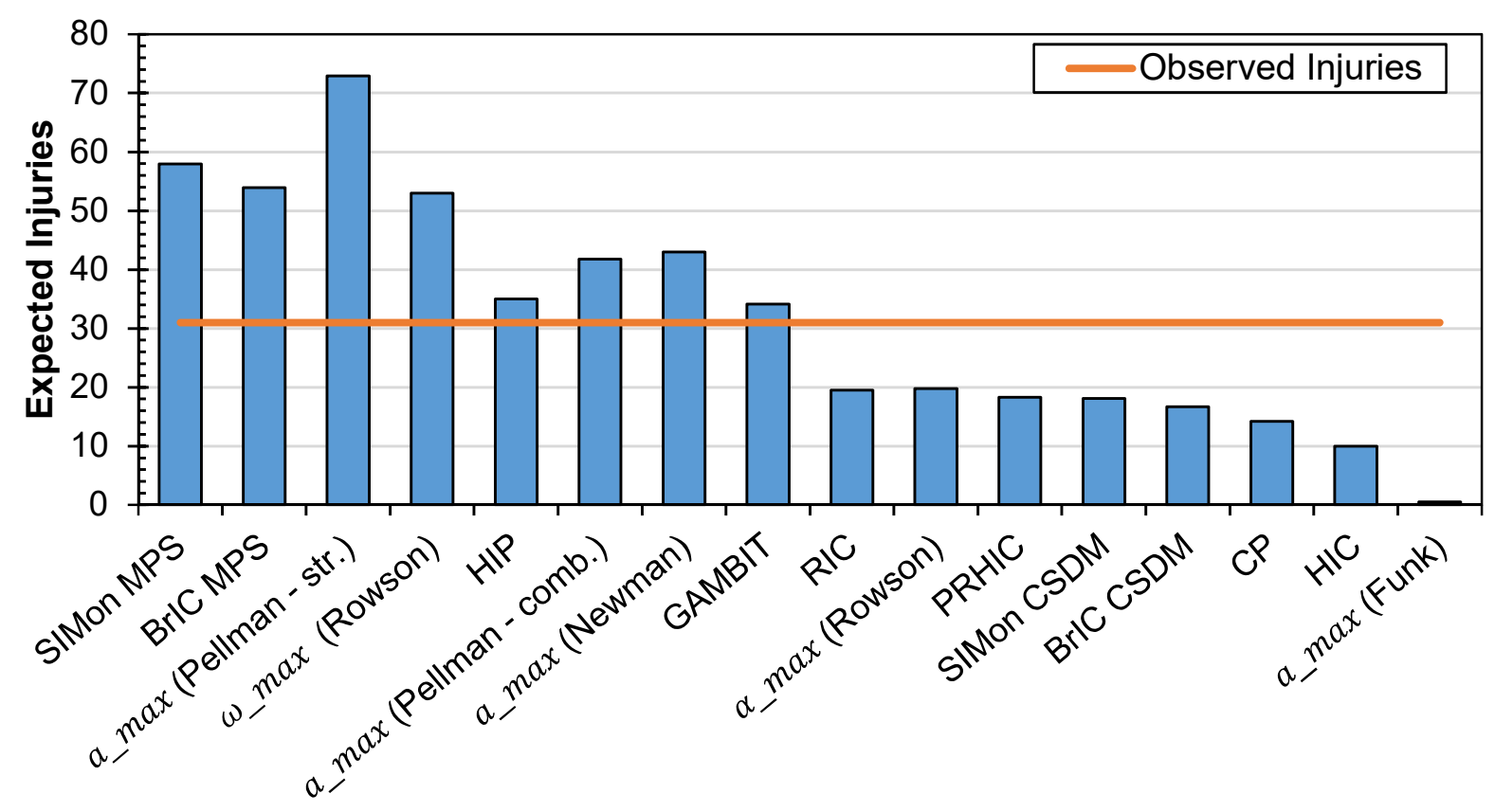

Figure 18. Expected number of injuries predicted for each injury risk function using only the most severe tests for each volunteer rather than all $335 \mathrm{HRV}$ sled tests. The full dataset still had 31 observed concussions. 


\subsubsection{Discussion and Limitations}

Results for the expected number of injuries estimated by all 16 injury risk functions were inconsistent; the trends in risk curve performance, however, were similar using the entire dataset and using a subset of data based on the most severe volunteer sled data. For example, using the linear acceleration risk curve developed by Funk would estimate less than 1 injury out of 31 observed injuries. On the other hand, the Pellman struck curve estimated 105 expected injuries, or $24 \%$ of the total dataset, compared to $7 \%$ observed injuries in the data $(59 \%$ of the subset using the most severe cases, compared to $25 \%$ ). The other linear acceleration risk curves (Pellman combined and Newman) had closer estimates to the observed number of injuries. The Pellman combined curve estimated $11 \%$ of injuries over the full dataset and $34 \%$ in the smaller cohort; the Newman risk function calculated $45(10 \%)$ and $43(35 \%)$ expected injuries from the total and smaller datasets, respectively.

Similarly, the BrIC CSDM risk curve would predict approximately 18 AIS 2+ injuries in the entire data $(4 \% ; 17$ injuries or $13 \%$ of the smaller subset of data) while the MPS-based BrIC injury risk function had one of the highest numbers of expected injuries (122 or 27\% of the full dataset and 54 or $13 \%$ of the smaller cohort). The large difference between these two curves can likely be attributed to the large number of $0 \%$ injury risk probabilities estimated by the CSDMbased BrIC curve; as previously mentioned, this curve associates a $0 \%$ probability of injury to every BrIC value below 0.523 due to the offset value $(b)$ in its formulation (Equation 3 ).

A concern of including the less severe volunteer sled exposures is that these could inflate the number of expected injuries by having such a large number of impacts. Further, these less severe sled tests are comprised from the same 22 volunteers, therefore, it would be surprising if the volunteers were injured on a less severe impact and not on a higher severity sled run. To 
account for this, expected injuries were calculated using only the most severe sled impacts. As mentioned above, the trends using both the full dataset and the smaller subset were similar. However, by comparing differences in the expected injuries predictions using the full dataset and the most severe runs, the average injury risk probability can be calculated for the less severe HRV sled tests. That is, by calculating the difference in the expected number of injuries from both methods and dividing the difference by the number of sled tests not used in the smaller data cohort, the average injury probability of these sled tests can be found. For most injury risk functions, the less severe sled tests had an average injury risk value of around or less than $1 \%$. However, injury risks calculated from the Pellman struck maximum linear acceleration and peak angular velocity curves averaged $10 \%$ for the less severe sled impacts. The MPS-based risk curves (MPS100 and BrIC) each had average injury probabilities of 23\% and $21 \%$. Given that these were volunteer sled tests which must be designed with little to no likelihood of injury, it seems likely that an average injury probability of $10 \%$ or greater is indicative of an overpredictive injury risk function.

\subsection{Discussion}

In the previous four sections, the ability of 16 head and brain injury risk functions were assessed on their ability to correctly predict injury outcomes over the full dataset using 6 DOF

head kinematic responses from more than 400 volunteer sled tests and football reconstructions. Numerous kinematic- and strain-based injury criteria and their associated risk functions have been developed to predict the risk brain injury. Having an accurate injury risk assessment tool can have a significant effect in head protection and the development of safety systems such as helmets and automobile restraints. Therefore, the accuracy of the risk functions for correctly assessing brain injury risks over a wide range of severities is of utmost importance. In this 
chapter, numerous risk assessment curves were evaluated using several statistical analysis techniques with mixed results.

For example, while CP had the best AUC value, it had the second worst correlation with both MPS95 and

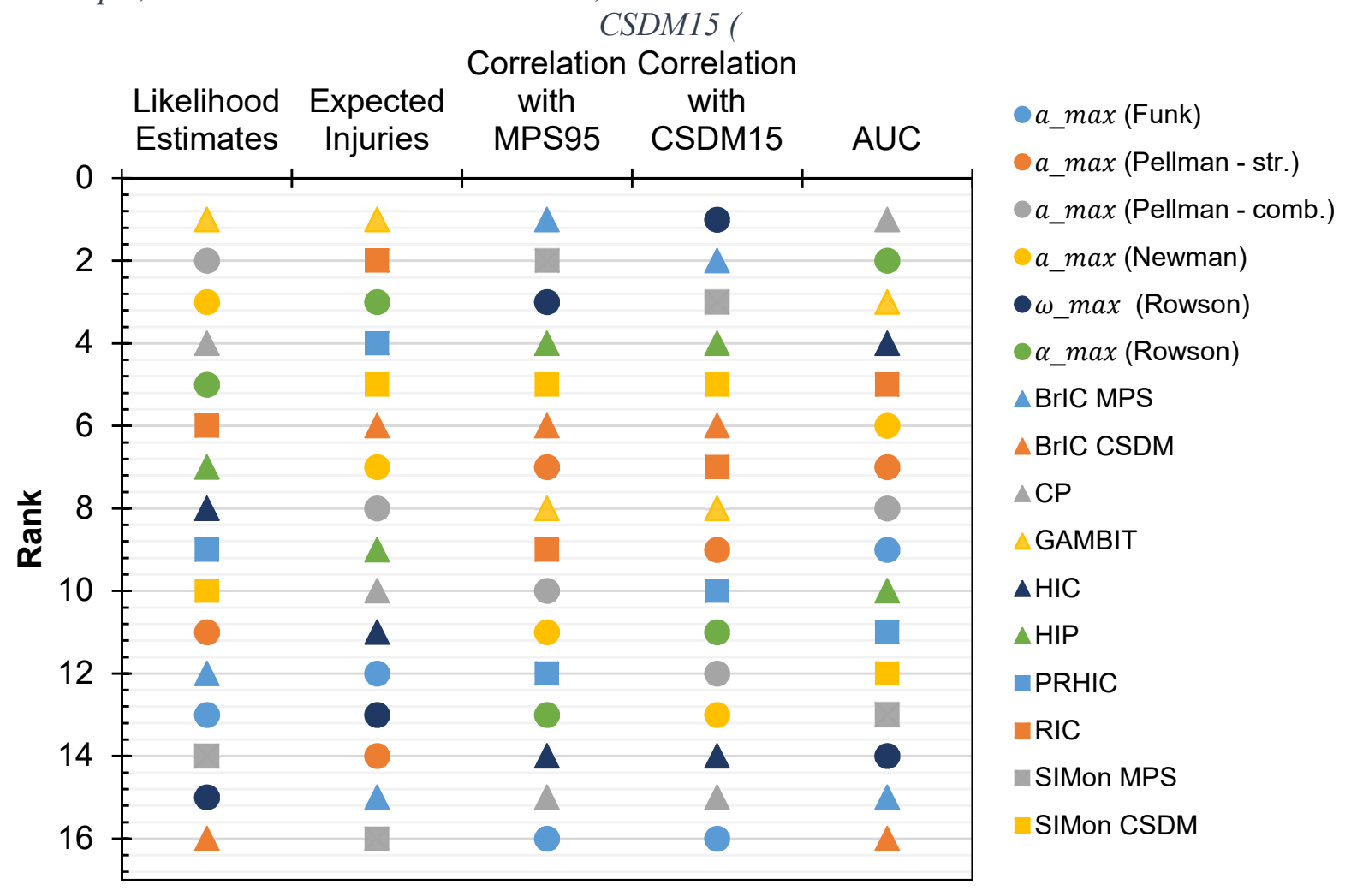

Figure 19). Alternatively, while the MPS-based BrIC injury risk function had the strongest correlation with the MPS95 strain metric and the second strongest correlation with CSDM15, it grossly over-predicted the number of expected injuries with respect to the entire dataset and the smaller data subset of only the most severe volunteer sled runs. 


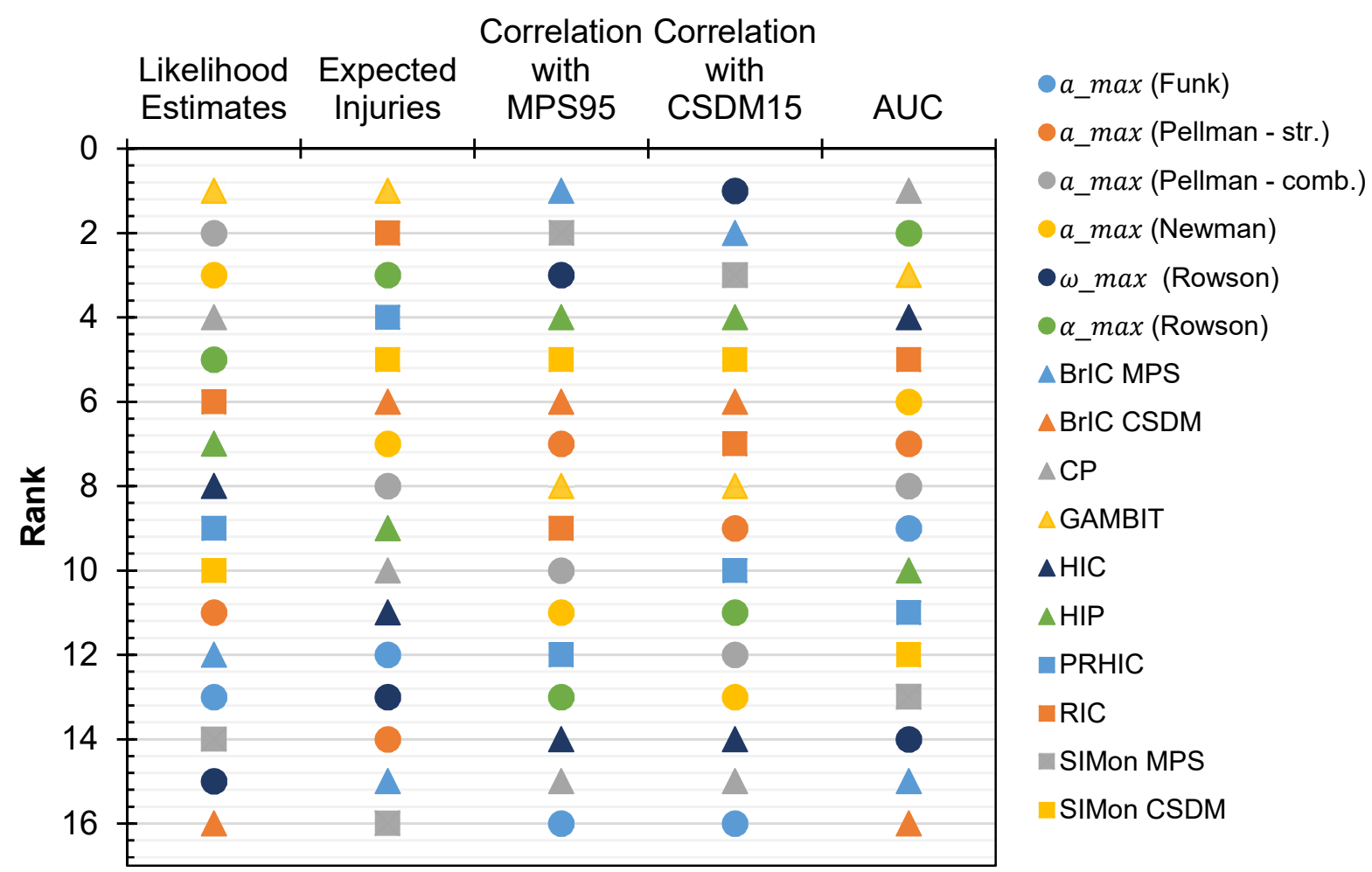

Figure 19. Injury risk function ranks with respect to each evaluation metric.

Results in literature also show mixed results. Hernandez et al. (2015) evaluated brain injury metrics (not injury risk functions) on their ability to predict mild traumatic brain injuries using head impact data from football, boxing, and martial arts. They found that metrics comprised of translational and rotational kinematic measures, such as GAMBIT and HIP, were among the best kinematic-based predictors of injury, as was maximum resultant angular acceleration. Change in peak angular velocity, BrIC, and PRHIC and translation-only metrics were less capable of predicting injury outcomes (Hernandez et al., 2015). Gabler et al. (2016a) used 660 head impacts from occupant and pedestrian crash tests and pendulum head impacts to assess the capability of fifteen kinematic-based injury metrics for predicting strain-based brain response. Correlations between the kinematic and strain-based metrics showed that rotationallybased metrics, particularly those derived from angular velocity, had the highest correlations with strain. Specifically, BrIC had one of the highest overall correlations. Metrics derived from linear 
acceleration were the least correlative with strains (Gabler et al., 2016a). While Hernandez et al. (2015) found that metrics incorporating 6 DOF kinematic measures were more capable of predicting injury, this study by Gabler et al. (2016a) suggested that rotational head kinematics are the most important parameters for brain injury criteria. It is worth noting that these studies did not assess the injury risk functions; rather, their results were drawn from analysis of head and brain injury risk metrics used as input to the risk functions.

Studies have also evaluated the efficacy of kinematic-based brain injury criteria using field data from motor vehicle crashes. Laituri et al. (2015) assessed brain injury risk functions by comparing injury risk probabilities from FE reconstructions to real-world injury rates obtained from the National Automotive Sampling System (NASS-CDS). They found that the AIS 2+ injury rates based on $\mathrm{HIC}$ and $\mathrm{CP}$ were more consistent with the real-world injury rates than the MPS-based BrIC injury risk function (Laituri et al., 2015). Another study also used the NASSCDS database to identify head injury rates in real-world automotive crashes and compared the crash configurations, vehicle damage, and occupant patterns to overlap crash tests from the Insurance Institute of Highway Safety (Mueller et al., 2015). They found injury probabilities estimated from HIC were closer to real-world estimates for AIS 3+ and 4+ injury severity levels than those predicted by BrIC (Mueller et al., 2015). Similarly, NHTSA's oblique frontal crash tests were used to predict BrIC injury risks and showed that the BrIC injury probabilities estimate higher rates of AIS $3+$ head injuries than currently seen in similar real-world crashes (Prasad et al., 2014).

While results are mixed in terms of injury risk function performance, if one risk metric and function had to be used, the strain-based injury risk functions are likely the best choice. Despite the CSDM risk curve estimating a $0 \%$ probability of injury for a concussive case and 
that the MPS injury risk function over-estimating the number of expected injuries, strain is the presumed mechanism of diffuse type brain injury; the strain-based injury risk functions had the best correlations with strain metrics (section 4.2). Therefore, the design of injury countermeasures and head protective equipment, which aim to reduce the probability of injury, should decrease the strain metrics. As mentioned in section 1.2.3, injury risk functions are monotonically increasing functions with injury probabilities increasing as the predictor variable increases. As such, regardless of the injury risk function used, reducing the strain metrics will also reduce the injury probability even if the injury risk function cannot correctly estimate injury outcome. Until there is an injury risk function that both matches the underlying injury mechanism and is capable of correctly estimating injury outcomes, strain metrics should be reduced as a means for reducing injury risk probability.

The reason for the disagreements seen in this thesis and in literature is likely due to the underlying data used in this thesis, as well as the data from which injury risk functions are developed. The HIC injury risk function was derived from PMHS data for skull fracture, but functional injuries such as concussion cannot be assessed using this type of data and without a physiologic response. Data from animal models, such as that used to develop the BrIC and strain injury risk functions, must be scaled to apply to humans, making it difficult to relate kinematic parameters and injury metrics to injury outcomes (Ommaya, 1985; Panzer et al., 2014). Some injury risk functions were derived from indirect measures of human head kinematics in the form of football impact reconstructions. However, these reconstructions were of significant hits, and these hits are not necessarily representative of the total exposure to head impact seen during the course of a football game or season. Rather, the selection bias of these reconstructions towards the concussive and significant impacts may cause injury risk curves derived from the data to 
over-predict the risk of injury (Broglio et al., 2010; Funk et al., 2007, 2012; Schnebel et al., 2007).

Several injury risk curves were developed using exposure data, yet were still unable to perform well across all evaluation metrics. These risk curves include the linear acceleration curve by Funk et al. (2007), Rowson et al.'s (2012) curves for peak angular acceleration and angular velocity, and the curve developed using CP by Rowson \& Duma (2013). For the linear acceleration curve and the $\mathrm{CP}$ curve (which includes peak resultant linear acceleration), it is also possible that these did not perform well due to their use of translational kinematics. Rotational measures of motion have been found to be better indicators of strains given that rotation is the simplest way to generate shear strain in the brain (Gabler et al., 2016a; Gennarelli et al., 1982; Holbourn, 1943). The peak angular velocity and acceleration curves, on the other hand, should be well-suited for predicting concussion according to the previous statement. However, these curves were developed using indirect measures of rotational motion. The angular acceleration term used as a predictor for the risk function was calculated using an equation with linear acceleration as the input; peak angular velocities were then calculated from the estimated angular acceleration terms in another linear equation (S. Rowson et al., 2012).

It should also be noted that the data used in this thesis for the assessment of the injury risk functions was also biased. The professional football reconstructions were again of significant and concussive hits, with the smallest magnitude at just $32 \mathrm{~g}$. Exposure data measured from the HIT system in collegiate football showed that $70 \%$ of impacts occur at magnitudes below $30 \mathrm{~g}$ (Funk et al., 2007). Similar results are found with the collegiate football reconstructions, although the magnitudes of these impacts were slightly more representative of the overall exposure data. Further, while the dataset in this thesis seems to include too many 
significant hits, the inclusion of 335 volunteer sled tests may also cause the dataset to over exaggerate the number of non-injurious impacts. As such, the overall exposure rate of the data may not be representative which could equally affect the assessments on the injury risk functions.

\subsection{Summary}

This chapter used several statistical techniques to evaluate injury risk functions, yet there was no overall consensus between these techniques that one risk function was more capable at predicting injury than the others. Further, literature shows that there is currently no consensus on a universally accepted injury metric and risk function. Studies disagree as to whether combination metrics or rotationally-based metrics are more capable or predicting the brain strain response (Gabler et al., 2016a; Hernandez et al., 2015). Studies also show that some injury risk functions may overestimate field-based injury rates (Laituri et al., 2015; Mueller et al., 2015; Prasad et al., 2014).

Reasons for disagreement were explored, especially as it relates to selection bias in both the development of the injury risk functions as well as selection bias within the dataset used for evaluation in this thesis. The next chapter explores the effects of selection bias and exposure rate through simulations in order to better illustrate how these may affect injury risk functions. The next chapter explores this idea through simulations to better illustrate the effect of selection bias and impact exposure. 


\section{CHAPTER 5: CASE-CONTROL DATA AND LOGISTIC REGRESSION}

\subsection{Introduction}

When injury risk curves are derived from case-control data, such as the professional football reconstructions, they may over-predict the absolute injury risk (Broglio et al., 2010; Funk et al., 2007, 2012; Schnebel et al., 2007). However, this type of data may be able to correctly estimate some parameters of the injury risk functions (Agresti, 2013). For example, logistic regression models derived from retrospective and case-control studies have been shown to correctly determine the relationship between an unknown explanatory variable and a known outcome (Agresti, 2013; Prentice \& Pyke, 1979). In terms of the injury risk functions from chapter 3, the coefficients on the predictor variables $\left(\beta_{1}, \beta_{2}\right.$, and $\beta_{3}$ in Equations 2,5 , and 8) found using case-controlled may be valid estimates in measuring the odds of having a positive outcome for a population. On the other hand, the intercept term $\left(\beta_{0}\right.$ in Equations 2,5 and 8$)$ is not a valid estimate because data have a fixed number of observed outcomes set by the case-control design (Agresti, 2013).

This characteristic of model parameters is only possible using logistic regression and the logit transformation (Equation 14; Agresti, 2013). When a logistic regression model is developed using the logit transformation, the regression coefficient is called the odds ratio and denotes the estimated increase in the log-odds of the outcome of the outcome per unit increase in the exposure value (Szumilas, 2010). Odds ratios can be used to compare the relative odds of an observed outcome given a predictor variable, and can determine association between a predictor variable and an outcome. While literature shows that this is a characteristic of logistic regression in case-control studies, it does not list requirements on the data sampling. This chapter explores 
possible limitations of this property through statistical simulations of data sampling in order to determine the necessity of exposure data in creating injury risk functions.

\subsection{Methods}

An overall distribution of head impact exposure in collegiate football impacts was created using real-time head acceleration data measured via the HIT. Funk et al. (2007) expressed this exposure data as a standard Weibull distribution and fitted the scale and shape parameters to the true distribution of impact magnitudes (scale $=24.41$, shape $=1.03$; Figure 20). For the simulations, this Weibull distribution was used to pick a random sample of peak resultant linear acceleration values to represent head impacts.
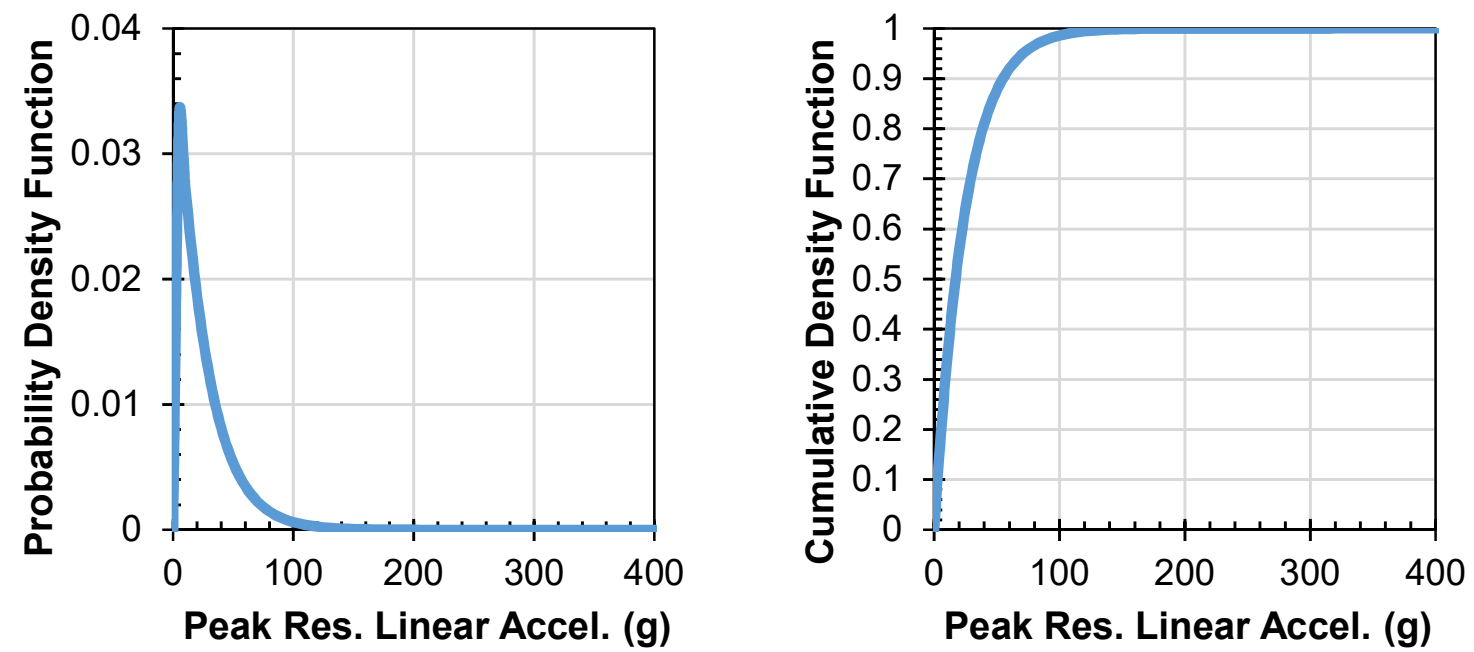

Figure 20. Weibull distribution fitted to the resultant head linear acceleration exposure in collegiate football impacts.

Injury outcomes had to be assigned to each of the sampled head linear accelerations; therefore, an injury risk function was defined to serve as a true risk curve. This curve was based on the peak resultant linear acceleration curve determined by Funk et al. (2007). The curve by Funk et al. was developed using real-time head exposure data measured via the HIT system as well as concussive and non-concussive football reconstruction data. Unlike other risk curves 
developed for peak linear acceleration, this curve includes unbiased estimates of impact exposure and is therefore likely to be more representative of absolute risk compared to the other linear acceleration curves. The injury risk function by Funk et al. was defined using a Weibull distribution, however, as previously mentioned, the ability to correctly approximate coefficients on the predictor variables only applies to logistic regression models. Therefore, a new logistic regression model was developed by fitting parameters to the Weibull curve that minimize the difference between the two models $\left(\beta_{0}=-5.31, \beta_{1}=.0149\right.$; Figure 21).

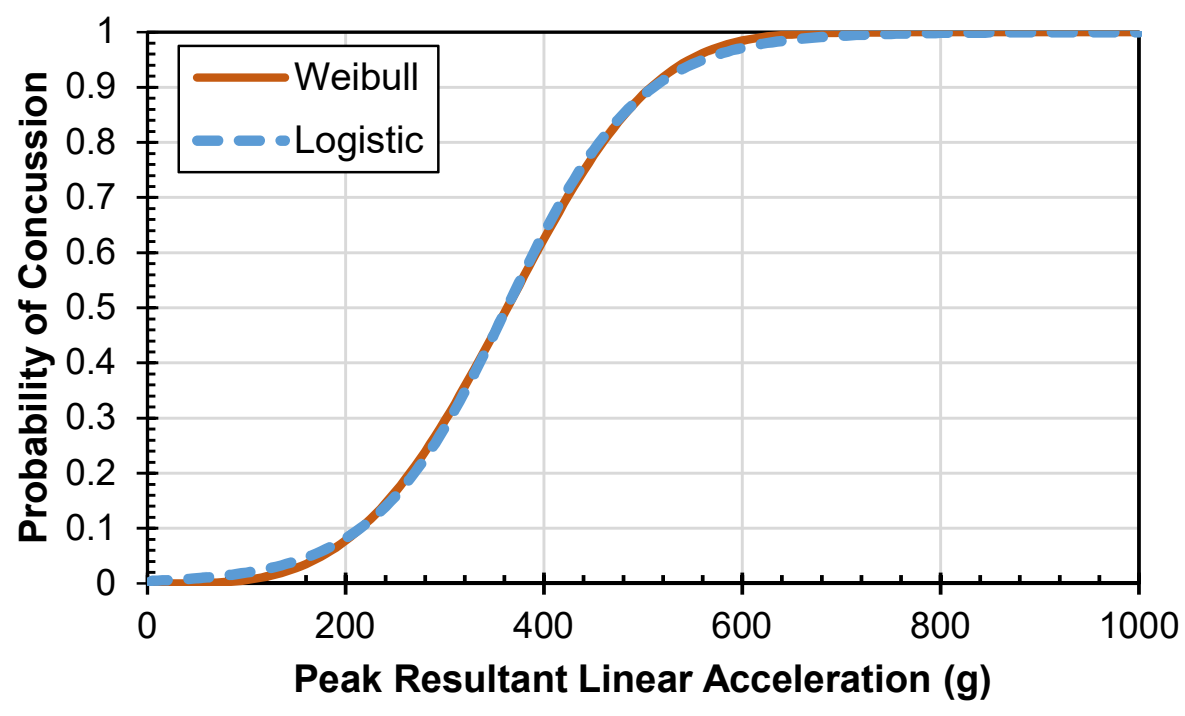

Figure 21. Plots of the original injury risk function developed by Funk et al. (2011) using a weibull distribution compared with its equivalent developed using logistic regression. Regression coefficients were chosen to mimize the difference between the two curves

For each sampled peak resultant linear acceleration value, a probability of injury was calculated using the defined true injury risk curve. Injury outcomes were then determined by sampling from the probability of injury risk. For example, a $200 \mathrm{~g}$ head impact was associated with an injury risk of about $10 \%$. Consequently, a vector was defined such that $10 \%$ of the values had an injurious outcome (i.e., a value of 1 ) and $90 \%$ represented a non-injurious outcome 
(i.e., a value of 0). A single value was randomly chosen from this vector to represent the $200 \mathrm{~g}$ head impact injury outcome.

Several situations were studied using these simulations. First, 1000 samples were taken from the distribution to ensure a similar fit to the true risk curve (case A). Next, a random sample of 60 significant hits was defined (case B). Significant hits were defined in this thesis as having peak resultant acceleration values above $35 \mathrm{~g}$; therefore, samples were randomly chosen from a truncated exposure distribution to ensure all values were above this threshold (Figure 22). The lower limit of $35 \mathrm{~g}$ was chosen based on the lower head acceleration values determined in professional football reconstructions (Pellman et al., 2003), as well as from thresholds previously used in the literature (Funk et al., 2007). When calculating injury outcomes, one sample had to be forced to have a positive injury outcome so that the injury risk curve would have a non-zero slope.

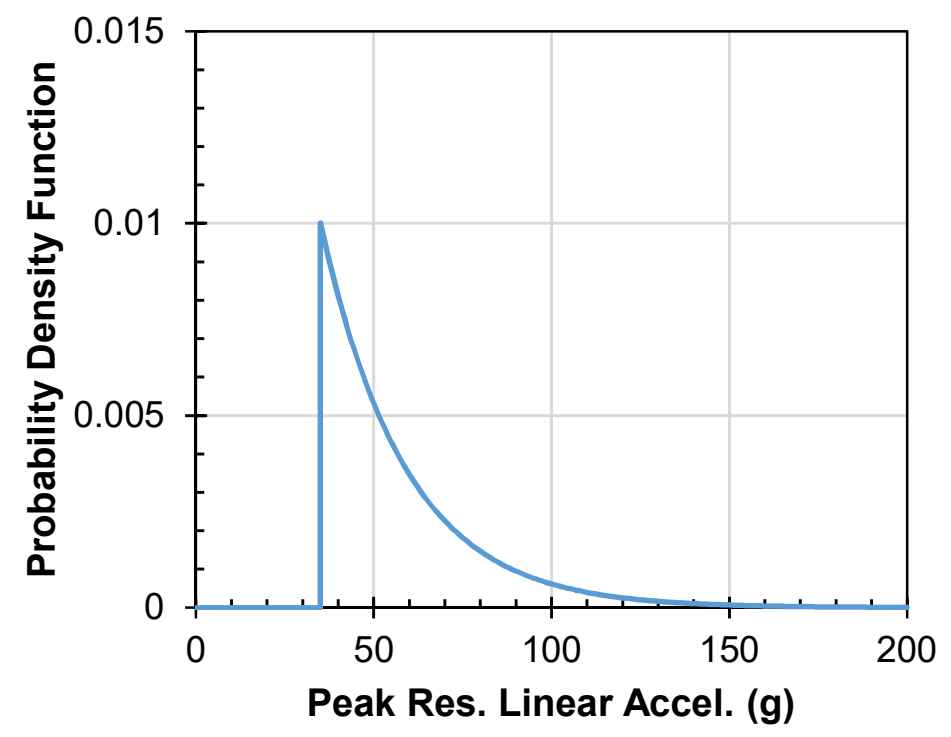

Figure 22. Exposure distribution used for sample scenarios $B$ and $C$, in which only significant hits (i.e., above $35 \mathrm{~g}$ ) were considered. The Weibull exposure distribution is defined using the same coefficients as in Figure 20. 
Third, a sample was created to replicate the professional football dataset in order to create a sample of selection bias (case C). The impacts in the professional football reconstruction dataset were originally chosen strictly because they were concussive or big hits, not because they were representative of the impacts seen over the course of a game or season. Head acceleration values were sampled as in case B with a lower limit of $35 \mathrm{~g}$ (Figure 22). Unlike case B (significant hits), however, the selection bias sample scenario aimed to have a higher percentage of concussive injury outcomes. Therefore, rather than sampling injury outcomes, these were assigned such that the first 25 samples were given an injurious outcome and the remaining 35 impacts were assigned a 0 (non-injurious).

Finally, case D still wanted to have a large number of concussive impacts, but aimed to have both the concussive and non-concussive impact distributions be representative of the impact exposure. As such, for case D, 10,000 samples were taken from the distribution and their injury outcomes were determined. This was split into a concussive subset (injury $=1$ ) and a nonconcussive subset; examples of the distributions of these can be seen in Figure 23. Values were then randomly sampled from both of these subsets 25 times so that the final sample set had 25 concussive impacts and 25 non-concussive impacts with their associated head linear accelerations. Samples were made with replacement to account for the fact that there may be less than 25 values in the concussive data subset. 


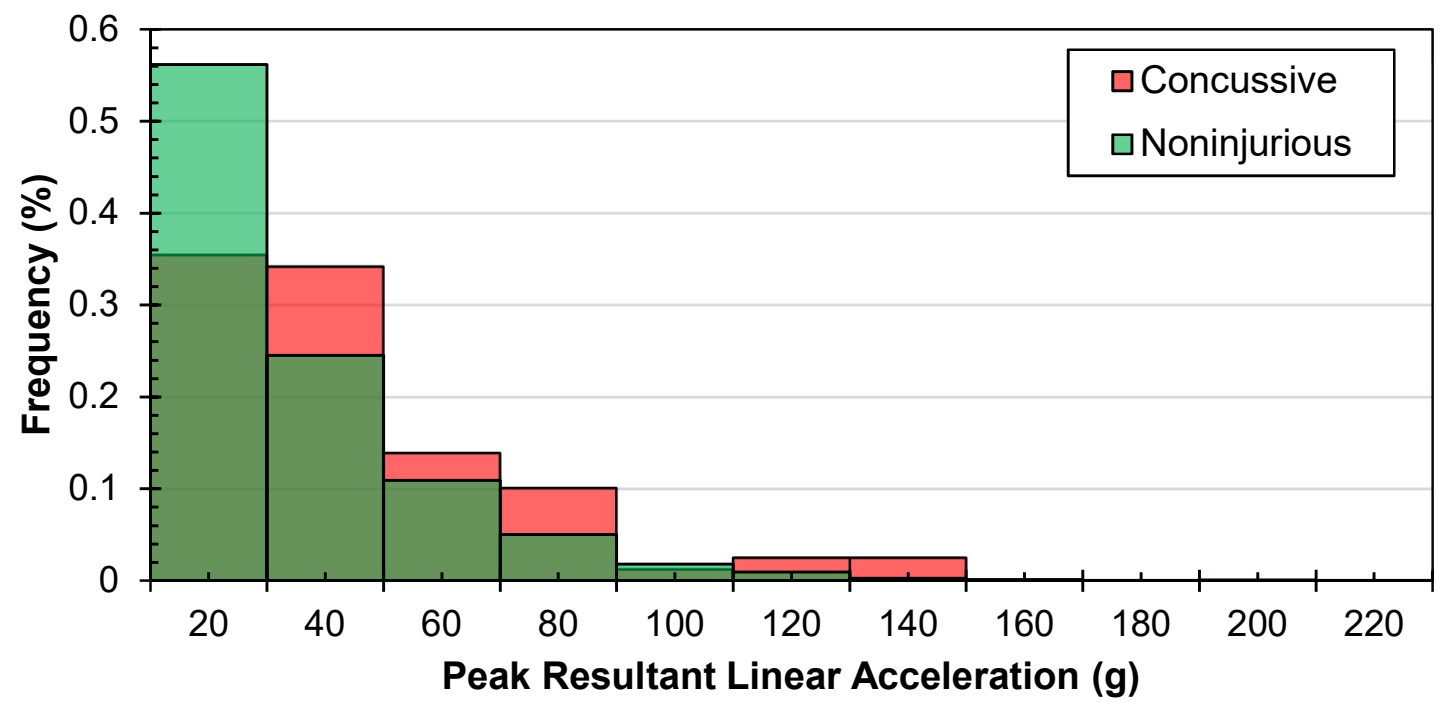

Figure 23. Distribution of the concussive and non-concussive impacts based on 10,000 samples.

After each sample in the scenarios described above, the data were fit with a logistic regression model in the form of Equations Equation 1 and Equation 2. One thousand samples were taken for each scenario in order to determine an average injury risk curve, as well as a $95^{\text {th }}$ percentile confidence interval. Both the median slope coefficient $\left(\beta_{1}\right)$ and the intercept value $\left(\beta_{0}\right)$ from the 1000 samples in each scenario were compared to the true risk curve to determine limitations on using case-control data to create an injury risk function representative of the absolute risk of the population.

\subsection{Results}

The injury risk curves (Figure 24) had a range of slope and intercept parameters, as described in Table 7 . The slope $\left(\beta_{1}\right)$ for case A, the 1000 sample set to ensure similar fit to the true risk curve, had a value of 0.0141 . This slope value fell within a 5\% error margin compared to the real slope value of 0.149 . The intercepts were also similar $(-5.29$ vs -5.31 for the true risk curve; $0.4 \%$ error). Case B created a logistic regression model using significant hits (i.e., head accelerations about $35 \mathrm{~g}$ ) and estimated a slope of 0.0054 (63.5\% error). The intercept value (- 
$4.22 ; 20.5 \%$ error) also varied with respect to the true risk curve's intercept term. The sample set in case C had a larger (and therefore steeper) slope value of 0.0437 (194.2\% error) and an incorrect intercept as well $(-2.97 ; 44.1 \%$ error). The case D intercept estimate had the largest percent error of all intercept terms $\left(-0.47 ; 91.1 \%\right.$ error), but its $\beta_{1}$ value of 0.0157 (5.6\% error) was much closer to that of the true risk curve's than cases B and C and was nearly identical in percent to the slope estimated by case A.

Table 7. Coefficients for the logistic regression models determined from each of the four sampling scenarios. Parentheses indicate 95\% confidence intervals.

\begin{tabular}{c|cc}
\hline & $\boldsymbol{\beta}_{0}$ & $\boldsymbol{\beta}_{1}$ \\
\hline True Risk & -5.31 & 0.0149 \\
Case A & $-5.29(-6.55,-4.49)$ & $0.0141(-0.190,0.031)$ \\
Case B & $-4.22(-12.99,9.86)$ & $0.0054(-0.339,0.101)$ \\
Case C & $-2.97(-5.42,-1.36)$ & $0.0437(0.168,0.089)$ \\
Case D & $-0.472(-1.18,0.061)$ & $0.0157(-0.003,0.042)$ \\
\hline
\end{tabular}

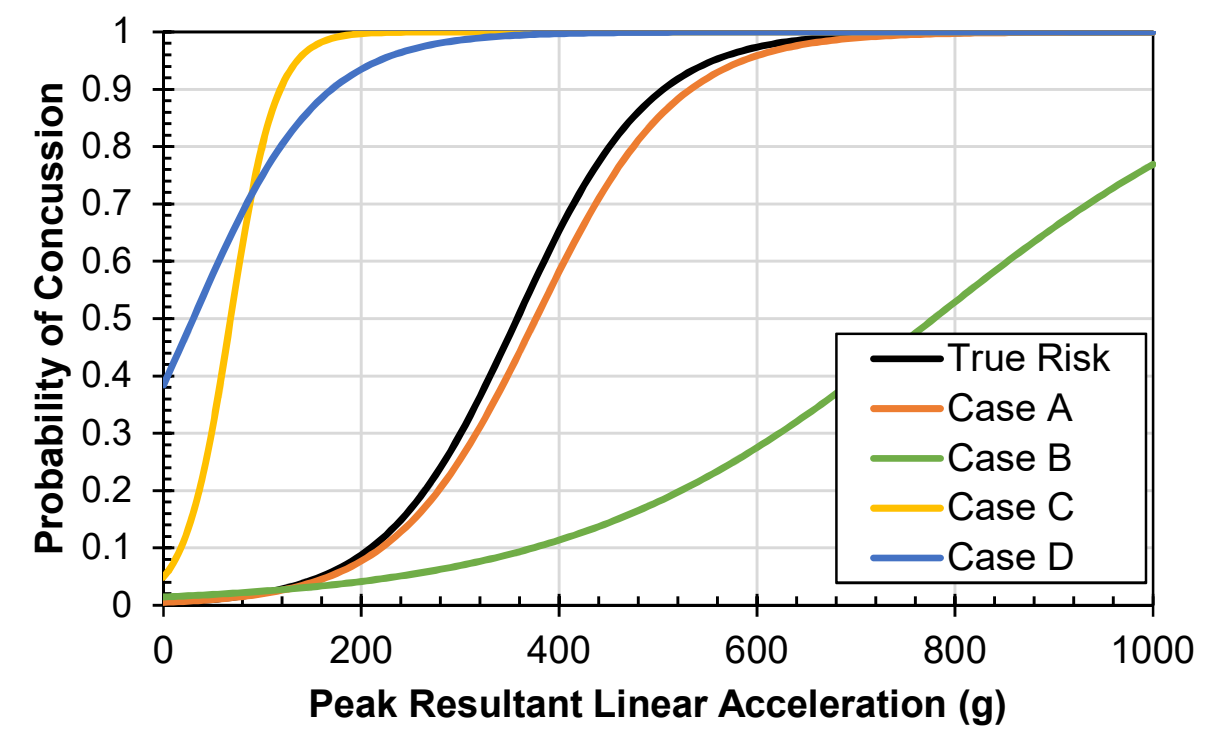

Figure 24. Injury risk curves determined by the four sampling scenarios, compared to the curve defined to represent true injury risk. 


\subsection{Discussion}

Plotting the curves created by these four scenarios shows that only case A recreated the predefined true risk curve with some accuracy (Figure 24). The scenario which only included significant hits (case B) resulted in a much shallower slope than that of the true risk curve; this can be attributed to the data only having 1 positive injury outcome. Even though this situation was defined to have head impacts resulting in accelerations above $35 \mathrm{~g}$, samples still had to be forced in order for there to be a single positive injury outcome. Further, these impacts still tended towards lower severities and were clustered just above the $35 \mathrm{~g}$ threshold due to the higher rate of exposure at this level of impact. In case $\mathrm{C}$, on the other hand, the assigned injury rates of 25 concussions versus 31 non-injurious impacts caused the slope to be much steeper than the true risk curve. The predefined injury rate $(45 \%)$ was much higher than that observed in both scenario A (about 1\%) and B (approximately $2 \%$ ). It was also higher than incidence rates reported in literature; Rowson et al. (2012) reported 0.726 concussions per 1000 impacts when considering underreporting of concussive injury and Lincoln et al. (2011) reported an incidence rate of 0.60 concussions per 1000 exposures in football, where an exposure is defined as one athlete participating in one game or practice.

The slope parameter estimated by case D, however, was very near to the slope of the true risk curve; recall that in case $\mathrm{D}$ an equal number of concussive and non-concussive impacts were sampled from exposure data to create the logistic regression model. This correct slope estimate was determined despite having a 50\% injury incidence rate. However, because the random sampling was representative of the underlying exposure curve, this type of case-control simulation could estimate the change in likelihood of concussion per unit change in head acceleration (Prentice \& Pyke, 1979). 
Perhaps the simplest explanation for case $\mathrm{D}$ being able to correctly estimate the odds ratio $\left(\beta_{1}\right)$ when cases $\mathrm{B}$ and $\mathrm{C}$ could not can be seen with a frequency table (Figure 25). In medical studies, the odds ratio is the ratio between the odds of developing a disease given exposure to the disease and the odds of developing the disease given non-exposure (Szumilas, 2010). In terms of the frequency table, this equates to the ratio between exposed cases and exposed controls (i.e., the probability of getting the disease given exposure) divided by the ratio between the number of non-exposed cases and the number of non-exposed controls (i.e., the probability of getting the disease given non-exposure). In other words, the odds ratio is:

$$
\mathrm{OR}=\frac{a / b}{c / d}
$$

Equation 16

or

$$
\mathrm{OR}=\frac{a / c}{b / d}
$$

Equation 17

\begin{tabular}{r|c|c|}
\multicolumn{1}{c}{} & \multicolumn{1}{c}{ Injured } & \multicolumn{1}{c}{ Uninjured } \\
\cline { 2 - 3 } Exposed & $\mathrm{a}$ & $\mathrm{b}$ \\
\cline { 2 - 3 } Unexposed & $\mathrm{c}$ & $\mathrm{d}$ \\
\cline { 2 - 3 } & &
\end{tabular}

Figure 25. Two-by-two frequency table used for calculating the odds-ratio.

To apply this to head impacts and concussions, a threshold of $100 \mathrm{~g}$ is used as an example to determine exposure. That is, if the head linear acceleration from impact exceeds $100 \mathrm{~g}$, the impact was considered as an exposed impact; if the acceleration falls below $100 \mathrm{~g}$, this case was considered unexposed. Therefore, $a, b, c$, and $d$ were defined as the areas shown in Figure 26. 

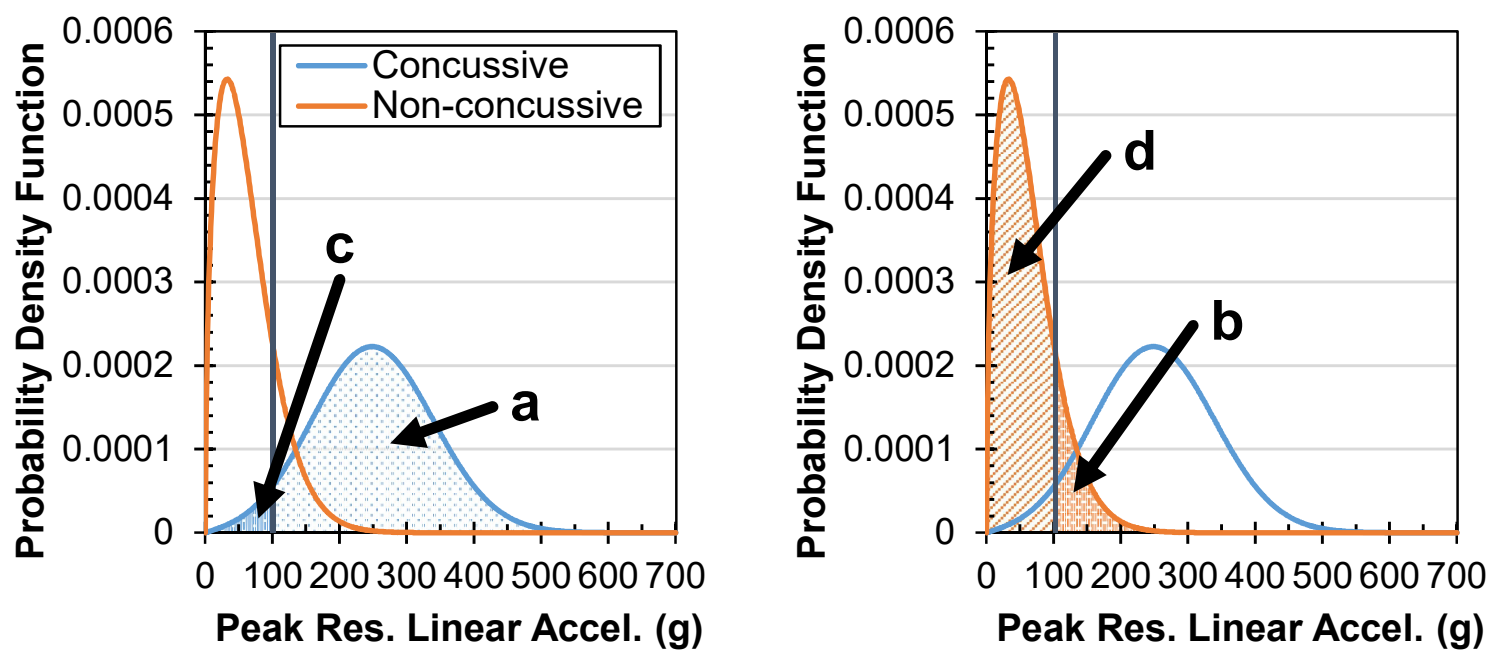

Figure 26. Definitions of $a, b, c$, and $d$ from the frequency table (Figure 25), as they may apply to injury and non-injury distributions in football head impacts.

For case A, the values of all four areas are at their maximums and equal to the true distribution of concussive and non-concussive impacts. On the other hand, in case B the values of $c$ and $d$ decreased due to the defined lower threshold of $35 \mathrm{~g}$ for significant hits. The area lost in $d$ was greater than that lost for $c$; this, in turn, decreased the odds ratio, or the slope value, by increasing the denominator in Equation 17. Similarly, case C decreased the values of $c$ and $d$ due to the defined lower threshold; however, by assigning injury risk values rather than randomly sampling changed the distributions themselves. In other words, the number of cases (concussions) is artificially inflated, causing both $a$ and $c$ to increase. The overall effect on the odds ratio is an increase in value. This equated to the increase in slope $\left(\beta_{1}\right)$ seen from the simulations.

In terms of the intercept, the only case capable of adequately estimating the intercept was case A, the scenario which randomly sampled 1000 head impact exposures and their associated injury outcomes. Case D had one of the closest slope parameter estimate, but also had the largest percent error for the intercept term (91.1\%). According to Agresti (2013), the intercept term is 
comprised of the true intercept value plus the log of the ratio of the probabilities of sampling a case versus sampling a control:

$$
\beta_{0}^{*}=\beta_{0}+\log \left(\frac{\rho_{1}}{\rho_{0}}\right)
$$

where $\beta_{0}$ is the true intercept of the population, $\rho_{l}$ is the probability of sampling a concussion, and $\rho_{0}$ is the probability of sampling a non-injurious case. If the probabilities of sampling a concussion $\left(\rho_{1}\right)$ and a non-injurious impact $\left(\rho_{0}\right)$ are the same, the log term would be 0 and only the true intercept term remains. Otherwise, if the sampling rate for cases is greater than that for controls, the intercept estimated is larger than the true intercept term. In case D, there were much fewer concussive cases to sample from than non-concussive data points; therefore, the probability of sampling an injurious case was lower than the probability of sampling a noninjurious case. This resulted in the intercept in case A to be smaller than the intercept term defined for true risk.

The effects of the various samples on the intercept and slope parameters in the various injury risk functions had a drastic effect on injury risk values such that a fifty percent probability of injury was associated with a range of head linear accelerations. Case D, with the smallest intercept and best estimation of the slope, associated $29.1 \mathrm{~g}$ with a $50 \%$ risk of injury while case B (significant hits), which had relatively poor estimates of the slope and intercept, assigned a $50 \%$ probability of injury to $778.5 \mathrm{~g}$. The true value defined to represent a $50 \%$ probability of injury was about $357.4 \mathrm{~g}$.

These injury risk values were drastically different from one another, and showed limitations in the methods currently used to develop injury risk functions for concussions and other types of diffuse head injuries. Excluding exposure data, while possibly capable of correctly estimating the relative change in injury risk per unit increase of predictor variable, could still 
create curves that express injury risks much too conservative or liberal compared to the true risk of a population depending on the sampling frequency of concussive and non-concussive injuries. Traffic safety can help illustrate this phenomenon. The risk of fatality in a crash at $30 \mathrm{mph}$ is about $10 \%$ despite crashes of this magnitude or less accounting for about half of all fatal crashes (Evans, 2004; Funk et al., 2007). Selection bias in sampling, such as choosing only significant hits, could incorporate too few or too many concussions compared to the true incidence and exposure rates, thereby estimating incorrect injury risk curve parameters.

Sample size may also effect the coefficients of the injury risk curve. In the scenarios above, 50-60 simulated head impacts were used in order to generate an injury risk function. These were repeated 1000 times so that an average risk function could be determined, however in real-life, researchers will not have the ability to create 1000 samples of size 60 to calculate a risk curve. Repeating the above process using various sample sizes found that, generally, the risk curve coefficients converged to those plotted in Figure 24 using 200 head impacts to for two of the sampling scenarios (cases $\mathrm{C}$ and $\mathrm{D}$ ). This was computed using measures of standard error; using the original sample size for cases $\mathrm{C}$ and $\mathrm{D}$, the standard error of the samples were between $6 \%$ and $8 \%$ for both $\beta_{0}$ and $\beta_{1}$. These standard error values dropped below $2 \%$ for both coefficients in all three cases when using 200 head impacts to generate an injury risk function and fell below $1 \%$ using 500 head impacts. For case B, which only included impacts above $35 \mathrm{~g}$ in peak resultant linear head acceleration, 600 head impacts were needed for similar standard errors (below 2\%).

While it may be possible to collect 200 or 600 head impacts via wearable sensors, reconstructing this number of head impact events, even with unbiased exposure data, is a large task. However, it could be possible to use weight the reconstructions in order to develop injury 
risk function rather than explicitly using unbiased exposure data. This is similar to the National Automotive Sampling System Crashworthiness Data System (NASS-CDS) maintained by NHTSA. The NASS-CDS is a comprehensive national traffic crash database that contains data weighted to represent all police reported motor vehicle crashes occurring in the United States during the year (Zhang \& Chen, 2013). Similarly, risk curves could be developed by determining weights based on the exposure to impact at various head impact severities. Funk et al. (2007) calculated concussion risk curves as a function of impact severity by normalizing the concussion incidence data (found via professional football reconstructions) to head impact exposure data (via HIT system data). Similarly, concussion incidence rates have been used to weight and combine concussive and non-concussive impact datasets in order develop injury risk functions (Rowson et al., 2012). This distribution weighting technique does not require a direct estimate of impact exposure, but may be create an injury risk curve more representative of the true risk.

Case D, which randomly sampled impacts from the concussive and non-concussive exposure, was able to correctly estimate the slope parameter of the injury risk function. However, it should be noted that this is only true if the injury risk function is calculated using logistic regression. When a logistic regression model is calculated using the logit transformation, the odds ratio can be found and used to compare the relative increase in probability as a result of a unit increase in the predictor variable (Szumilas, 2010). Unlike odds ratios, however, the effect of the predictor variable on an outcome determined from a different distribution (such as Weibull) using case-control data are not necessarily equal to the effect the predictor variable would have on the outcome of an entire population (Agresti, 2013). This is an important distinction, and a potential advantage of logistic regression models. As mentioned in chapter 1, the common procedure in literature for developing an injury risk function was to assign a form 
for the injury risk function a priori and then determine the parameters to maximize the function's fit to the data (e.g., using least-squares, maximum likelihood). Several statistical models have been used to define the form of injury risk functions; when the performance of four such distributions (log normal, logistic, normal, and Weibull) were compared, none were found to be consistently more appropriate for the datasets considered (Kent \& Funk, 2004). Given the similarity in performance between the distributions, it may be advantageous for developers of future injury risk functions to consider the ability of logistic models to correctly assess the odds ratio when weighing their distribution choices. This advantage may be lost if the logistic distribution is not appropriate underlying distribution of the population (DiDomenico \& Nusholtz, 2003), but it is likely that most experimental datasets do not collect sufficient data to justify which distribution is most appropriate for the larger population (Kent \& Funk, 2004). Researchers should also remember, regardless of the form of the injury risk function, that the samples of data used to develop the injury risk function will have profound effects on the final fit.

\subsection{Summary}

In this chapter, an exposure distribution and injury risk curve were defined to represent the true exposure to head impact and risk of concussion from head impact using data from the literature (Funk et al., 2007). Various samples of both injurious and non-injurious head impacts were taken using this distribution and injury risk function in order to explore how well different samples were able to recreate the parameters of logistic regression used to define the true injury risk curve. Of the three scenarios tested, two had slope and intercept parameters for the logistic regression model that had margins of error between $20 \%$ and $200 \%$. These two situations only considered significant hits, or in other words, hits with a magnitude of resultant head linear 
acceleration above $35 \mathrm{~g}$; hits of these magnitudes, however, account for only about $30 \%$ of the hits defined by the distribution. The third scenario randomly sampled 25 concussive and 25 nonconcussive impacts. This sample set was able to correctly estimate the slope, or the odds ratio, of the true injury risk curve. Based on knowledge about case-control studies and the logit transformation, this outcome was not surprising. This set was not, however, capable of recreating the intercept value of the true injury risk function because there were a controlled number of injurious and non-injurious outcomes.

This capability is a property of logistic regression models, and should be taken under consideration when developing injury risk functions. That is, researchers should consider both the sample of data they are using to build an injury risk curve as well as the form of the curve they are defining. Further, these simulations highlight the importance of exposure data when creating injury risk functions from head impacts. The scenario that was able to correctly estimate the slope parameter had sample distributions similar to that of the defined exposure distribution. The other situations were unable to reproduce any coefficient in the regression model due to their lack of ability to have the underlying exposure. At the same time, researchers should be aware that injury risk curves generated using case-control data are generally much too conservative compared to the real probabilities of injury and should know that without the correct exposure data for a given predictor variable, the risk of injury at severity levels where most injuries occur likely has a low risk of injury. 


\section{CHAPTER 6: CONCLUSIONS}

\subsection{Concluding remarks}

The goal of this thesis was to establish a verified set of human head kinematic data and to use the data to evaluate a variety of brain injury risk functions. To achieve this goal, a literature review of head kinematic data was conducted in chapter 2 in order to illustrate the availability of

these data as well as outline different manners of directly and indirectly measuring human head kinematics. These included laboratory tests using human research volunteers, the use wearable sensors on contact sport athletes to collect data from potentially injurious impacts, and computational and laboratory reconstructions of injurious head impact events. After understanding the types of data available in literature, specific data sources were chosen for use in a dataset of head impact kinematics.

In chapter 3, the head kinematic data collected in chapter 2 were assessed in order to establish a consistent dataset. Consistent was defined to mean that all data had valid sensor measurements, that the measured kinematics were representative of the motion from the actual impact (either sled test or reconstruction), that injury diagnoses only consisted of concussions, and that complete 6 DOF data were processed in the same manner and coordinate system. Once the data were properly assessed and processed, they were used to calculate various kinematic injury metrics and their associated injury risk probabilities. Distributions of both the kinematic parameters and all kinematic metrics showed a distribution of values with considerable overlap between the concussive and non-concussive injury outcomes. The 6 DOF kinematic data were also used as input to two FE models in order to calculate tissue-level brain strains as a result of each impact. Strain-based injury metrics were calculated from each FE simulation, as well as their associated injury risk values. 
Following the calculation of each injury risk probability, these values were used in chapter 4 to evaluate the efficacy of each of the 16 injury risk functions. Their discriminatory capability was assessed through correlations with strains as well as via ROC curves. All risk curves showed good diagnostic ability, with AUC values greater than 0.8 . Strain correlations were also decent, with risk functions based on rotational injury metrics showing better correlation values than combination metrics and translational metrics. Each injury risk function was also assessed by calculating expected injuries and through maximum likelihood estimates. The number of expected injuries was compared to the number of observed injuries in the dataset $(n=31)$ and showed that most injury risk functions were incapable of correctly estimating the number of injuries; the lone exception to this was the risk curve developed for GAMBIT. Likelihood estimates were also used to rank the injury risk functions based on their ability to correctly estimate injury outcomes over the entire database. Results from all four evaluation tools were mixed results as to which injury risk function performed best. Reasons for this were explored, including the necessity of exposure data for creating injury risk functions.

Studies have previously noted that in order to properly estimate absolute injury risk, unbiased exposure data is required. The need for exposure data, as well as strengths and limitations on using case-control studies to generate injury risk functions, was explored in chapter 5. It was shown that using case-control data and logistic regression can estimate the slope parameter if samples of both cases (concussions) and controls (non-injuries) are representative of the true exposure distribution of impacts. Other scenarios, such as only sampling significant hits, can result in risk curves drastically different from that of the true risk of the entire population. These various situations were demonstrated in order to show researchers to be wary of injury risk curves derived without exposure data, as they may over-exaggerate the risk of injury. Despite the 
issues with the risk functions, this thesis provides a verified data set that is a necessary step for injury risk function evaluation.

\subsection{Contributions}

A primary contribution of this thesis is the establishment of a verified dataset of human head kinematic data. These data, as shown in the thesis, are a necessary step for evaluating injury risk functions. The data can be used to validate current injury risk functions and can also be used as a basis for future head injury criteria or to establish injury assessment reference values. Using these data in addition with future data as it becomes available can mitigate the need to develop thresholds from scaled animal data, which are highly speculative given the need for scaling; from ATDs, which have questionable biofidelity in all impact scenarios; and from PMHS, which lack a physiologic response needed to diagnose concussion.

A second contribution is the demonstration of the importance of exposure data in creating injury risk functions. As shown in chapter 5, the parameters of the injury risk function were largely dependent on the underlying data used for its development. When data are chosen based on their injury outcome or due to the presumed magnitude of the hits, the risk function may be far too conservative compared to the real risk of the population. As an example, most car crashes are low severity impacts, therefore the incidence of injury at this crash magnitude is amplified despite there being a relatively low-risk of death or injury at these magnitudes. The same applies to head impacts, and future work in the development of head and brain injury risk functions needs to take this into account.

\subsection{Future research directions}

Future studies should attempt to gather more data regarding human head kinematics. The dataset established in this thesis accounts for 443 head impact scenarios and is a necessary step 
for risk function evaluation; however these are only football impacts (short duration, high acceleration) impacts and low-severity, volunteer sled impacts (long duration, low acceleration). Researchers should collect data from other head impact conditions that cover a wider range of magnitudes and durations.

Additionally, future work should look to continue characterizing exposure to head impacts. Due to selection bias in case-control studies, absolute injury risk curves derived from these data over predict the risk of injury (Broglio et al., 2010; Funk et al., 2007, 2012; Mihalik et al., 2007; B. Rowson et al., 2015). Funk et al. (2007) note that the key to obtaining unbiased head impact exposure data is to record all head impacts experienced. As wearable sensor technology becomes more available and more accurate, this data can be incorporated into the development and assessment of injury risk functions, as well as into this dataset of human head kinematics such that it better reflects the exposure distribution.

Researchers could attempt to evaluate other risk functions. Various other injury risk functions exist that use both kinematic and strain-based predictor variables (e.g., Funk et al., 2007; Newman et al., 2000a; Pellman et al., 2003; Zhang et al., 2004). These were not assessed in this thesis, however if assessed they may be found to be more capable of predicting injury risk than the curves evaluated in this thesis.

Finally, this thesis acknowledges that logistic regression models can correctly estimate the relative increase in injury probability based on a unit increase in the predictor variable. However, no work was done to investigate how to change the intercept parameter such that it better reflects the full population. Future studies could look into what kind of data is needed in order to adjust injury risk curves to represent a true risk curve. 


\subsection{Limitations}

Sixteen brain injury risk functions were evaluated in this thesis, but others exist. For example, Pellman et al. (2003) also published risk curves for HIC, SI, angular acceleration, angular velocity, and change in linear velocity and Zhang et al. (2004) developed injury risk functions using angular acceleration and shear strain in the brainstem as predictor variables for concussion. Such curves were not evaluated in this thesis and could be assessed in the future.

Understanding both the concept and definition of concussion and mild traumatic brain injury was vital in interpreting the results. The volunteer sled tests conducted by NBDL were conducted when concussion was defined by a loss of consciousness, and the definition of concussion has changed since then. Modern definitions have a wider range of symptoms and do not require loss of consciousness. All medical reports from the NBDL indicated that symptoms were mild and transient, however little effort was made at the time of the tests to distinguish symptoms as being a musculoskeletal or a neurological issue (Sanchez et al., 2017a). The diagnoses for the professional football players were more in tune with contemporary definitions of concussion, however there were still some variations in symptoms (Pellman et al., 2004b; S. Rowson et al., 2012). A secondary issue with injury definition was that not all injury risk functions were developed to predict concussion. BrIC, HIC, MPS100, and CSDM25 all had injury risk curves developed based on AIS codes. Concussion does not have an exact equivalency for concussion, but AIS 2+ curves were used to represent this type of injury. The criterion for an AIS 2 concussion is defined as a loss of consciousness less than 1 hour, however not all concussions in this dataset saw loss of consciousness.

Selection bias is always a concern with volunteer test data and can also be an issue with contact sport athletes. The subjects used in this analysis were military personnel and collegiate 
and professional football players; these subjects are not representative of the entire population and it is possible that this cohort of volunteers has a higher threshold for concussion than other healthy male subjects of a similar age.

No attempt was made to evaluate the fidelity of the football reconstructions with respect to the on-field impacts they were meant to recreate. The researchers of the professional football reconstructions aimed to recreate the relative impact velocity, with Newman et al. (2005) estimating errors up to $11 \%$ for the closing velocity. No attempt was made in the reconstructions to mimic the pre-impact motion of any of the players. For the collegiate football reconstructions, the criteria was to recreate head peak resultant kinematic parameters within $5 \%$ of the measured values (B. Rowson et al., 2017a, 2017b, 2017c). The use of ATDs in both of these reconstructions also includes inherent assumptions about the biofidelity of the surrogate; neck tensing, and post-impact kinematics could not be directly quantified using the game footage for the professional football reconstructions; further, the collegiate reconstructions only had $35 \mathrm{~ms}$ of post impact linear accelerations. No assessment was made for either on the representativeness of the reconstructed ATD head motion.

Brain deformations are believed to be the underlying mechanism for diffuse type injuries such as concussions, and as such the assumption is made in this thesis that strain is related with injury. This assumption relies heavily on the predictive capacity of the FE models. GHBMC and SIMon have both been validated for various intracranial responses including brain deformations, but it is possible that the durations and severities of these impacts fall outside the range of experimental data used to validate these models. Finally, while FE models are generally wellsuited for studying tissue deformation due to head impact, different head and brain models have been shown to predict different strain responses using the same kinematic input (Ji et al., 2014). 


\section{REFERENCES}

Abel, J. M., Gennarelli, T. A., \& Segawa, H. (1978). Incidence and severity of cerebral concussion in the rhesus monkey following sagittal plane angular acceleration. SAE Technical Paper.

Adams, J. H., Mitchell, D. E., Graham, D. I., \& Doyle, D. (1977). Diffuse brain damage of immediate impact type: its relationship to 'primary brain-stem damage' in head injury. Brain, 100(3), 489-502. https://doi.org/10.1093/brain/100.3.489

Agresti, A. (2013). Categorical data analysis (3rd ed). Hoboken, NJ: Wiley.

Allen, M. E., Weir-Jones, I., Motiuk, D. R., Flewin, K. R., Goring, R. D., Kobetitch, R., \& Broadhurst, A. (1994). Acceleration Perturbations of Daily Living. Spine, 19(11), 12851290.

Allison, M. A. (2015). The performance of helmet-based kinematic measurement systems: Importance for mild traumatic brain injury prevention (Ph.D.). University of Pennsylvania, United States -- Pennsylvania. Retrieved from https://search.proquest.com/docview/1699088557/abstract/501AA232A33E4679PQ/1

Allison, M. A., Kang, Y. S., Maltese, M. R., Bolte, J. H., \& Arbogast, K. B. (2015). Measurement of Hybrid III Head Impact Kinematics Using an Accelerometer and Gyroscope System in Ice Hockey Helmets. Annals of Biomedical Engineering, 43(8), 1896-1906. https://doi.org/10.1007/s10439-014-1197-z

Altman, D. G. (1990). Practical statistics for medical research. CRC press.

Arbogast, K. B., Balasubramanian, S., Seacrist, T., Maltese, M. R., Garcia-Espana, J. F., Hopely, T., ... Tanji, H. (2009). Comparison of kinematic responses of the head and spine for children and adults in low-speed frontal sled tests. Stapp Car Crash Journal, 53, 329.

Arbogast, K. B., Mathews, E. A., Seacrist, T., Maltese, M. R., Hammond, R., Balasubramanian, S., ... Higuchi, K. (2012). The effect of pretensioning and age on torso rollout in restrained human volunteers in far-side lateral and oblique loading. Stapp Car Crash Journal, 56, 443.

Arndt, S. R., Cargill, R. S., \& Hammoud, P. E. S. (2004). Head Accelerations Experienced during Everyday Activities and While Riding Roller Coasters. Proceedings of the Human Factors and Ergonomics Society Annual Meeting, 48(16), 1973-1977. https://doi.org/10.1177/154193120404801638

Auer, C., Schonpflug, M., Beier, G., \& Eisenmenger, W. (2001). An analysis of brain injuries in real world pedestrian traffic accidents by computer simulation reconstruction. In Proc. Int. Soc. Biomechanics XVIIIth Congress (pp. 680-686).

Bandak, F. A. (1995). On the mechanics of impact neurotrauma: a review and critical synthesis. Journal of Neurotrauma, 12(4), 635-649. 
Bandak, F. A., Zhang, A. X., Tannous, R. E., DiMasi, F., Masiello, P., \& Eppinger, R. H. (2001). Simon: a simulated injury monitor; application to head injury assessment. In Proceedings: International Technical Conference on the Enhanced Safety of Vehicles (Vol. 2001, p. 7 p.-7 p.). National Highway Traffic Safety Administration.

Barrett-Connor, E., \& Bush, T. L. (1991). Estrogen and coronary heart disease in women. Jama, 265(14), 1861-1867.

Beckwith, J. G., Greenwald, R. M., \& Chu, J. J. (2012). Measuring head kinematics in football: Correlation between the head impact telemetry system and hybrid III headform. Annals of Biomedical Engineering, 40(1), 237-248. https://doi.org/10.1007/s10439-011-0422-2

Beckwith, J. G., Greenwald, R. M., Chu, J. J., Crisco, J. J., Rowson, S., Duma, S. M., ... Collins, M. W. (2013a). Head Impact Exposure Sustained by Football Players on Days of Diagnosed Concussion: Medicine \& Science in Sports \& Exercise, 45(4), 737-746. https://doi.org/10.1249/MSS.0b013e3182792ed7

Beckwith, J. G., Greenwald, R. M., Chu, J. J., Crisco, J. J., Rowson, S., Duma, S. M., ... Collins, M. W. (2013b). Timing of Concussion Diagnosis Is Related to Head Impact Exposure Prior to Injury: Medicine \& Science in Sports \& Exercise, 45(4), 747-754. https://doi.org/10.1249/MSS.0b013e3182793067

Beeman, S. M., Kemper, A. R., Madigan, M. L., \& Duma, S. M. (2011). Effects of Bracing on Human Kinematics in Low-Speed Frontal Sled Tests. Annals of Biomedical Engineering, 39(12), 2998.

Beeman, S. M., Kemper, A. R., Madigan, M. L., Franck, C. T., \& Loftus, S. C. (2012). Occupant kinematics in low-speed frontal sled tests: Human volunteers, Hybrid III ATD, and PMHS. Accident Analysis \& Prevention, 47, 128-139. https://doi.org/10.1016/j.aap.2012.01.016

Begeman, P., \& Melvin, J. (2002). Mathematical Modeling of Crash-Induced Dynamic Loads on Race Car Drivers. https://doi.org/10.4271/2002-01-3305

Belmont Report. (1979). The Belmont Report: Ethical principals and guidelines for the protection of human subjects of research. Department of Health, Education and Welfare.

Bohlin, N. I. (1964). Studies of three-point restraint harness systems in full-scale barrier crashes and sled runs. SAE Technical Paper.

Bonita, R., Beaglehole, R., \& Kjellström, T. (2006). Basic epidemiology. World Health Organization.

Brainard, L. L., Beckwith, J. G., Chu, J. J., Crisco, J. J., Mcallister, T. W., Duhaime, A.-C., ... Greenwald, R. M. (2012). Gender Differences in Head Impacts Sustained by Collegiate Ice Hockey Players: Medicine \& Science in Sports \& Exercise, 44(2), 297-304. https://doi.org/10.1249/MSS.0b013e31822b0ab4 
Broglio, S. P., Eckner, J. T., Martini, D., Sosnoff, J. J., Kutcher, J. S., \& Randolph, C. (2011). Cumulative Head Impact Burden in High School Football. Journal of Neurotrauma, 28(10), 2069-2078. https://doi.org/10.1089/neu.2011.1825

Broglio, S. P., Eckner, J. T., Surma, T., \& Kutcher, J. S. (2011). Post-Concussion Cognitive Declines and Symptomatology Are Not Related to Concussion Biomechanics in High School Football Players. Journal of Neurotrauma, 28(10), 2061-2068. https://doi.org/10.1089/neu.2011.1905

Broglio, S. P., Martini, D., Kasper, L., Eckner, J. T., \& Kutcher, J. S. (2013). Estimation of Head Impact Exposure in High School Football: Implications for Regulating Contact Practices. The American Journal of Sports Medicine, 41(12), 2877-2884. https://doi.org/10.1177/0363546513502458

Broglio, S. P., Schnebel, B., Sosnoff, J. J., Shin, S., Feng, X., He, X., \& Zimmerman, J. (2010). Biomechanical Properties of Concussions in High School Football: Medicine \& Science in Sports \& Exercise, 42(11), 2064-2071. https://doi.org/10.1249/MSS.0b013e3181dd9156

Broglio, S. P., Sosnoff, J., Shin, S., He, Z., Alcaraz, C., \& Zimmerman, J. (2009). Head impacts during high school football: a biomechanical assessment. Journal of Athletic Training, 44(4), 342-349. https://doi.org/10.4085/1062-6050-44.4.342

Brolinson, P. G., Manoogian, S., McNeely, D., Goforth, M., Greenwald, R., \& Duma, S. (2006). Analysis of linear head accelerations from collegiate football impacts. Current Sports Medicine Reports, 5(1), 23.

Broshek, D. K., Kaushik, T., Freeman, J. R., Erlanger, D., Webbe, F., \& Barth, J. T. (2005). Sex differences in outcome following sports-related concussion. Journal of Neurosurgery, 102(5), 856-863.

Bussone, W. R., \& Duma, S. M. (2010). The effect of gender and body size on angular accelerations of the head observed during everyday activities-biomed 2010. Biomedical Sciences Instrumentation, 46, 166-171.

Cadet, J. L., Ladenheim, B., Baum, I., Carlson, E., \& Epstein, C. (1994). CuZn-superoxide dismutase (CuZnSOD) transgenic mice show resistance to the lethal effects of methylenedioxyamphetamine (MDA) and of methylenedioxymethamphetamine (MDMA). Brain Research, 655(1), 259-262.

Camarillo, D. B., Shull, P. B., Mattson, J., Shultz, R., \& Garza, D. (2013). An instrumented mouthguard for measuring linear and angular head impact kinematics in american football. Annals of Biomedical Engineering, 41(9), 1939-1949. https://doi.org/10.1007/s10439-013-0801-y

Campbell, K. R., Warnica, M. J., Levine, I. C., Brooks, J. S., Laing, A. C., Burkhart, T. A., \& Dickey, J. P. (2016). Laboratory Evaluation of the gForce Tracker ${ }^{\mathrm{TM}}$, a Head Impact 
Kinematic Measuring Device for Use in Football Helmets. Annals of Biomedical Engineering, 44(4), 1246-1256. https://doi.org/10.1007/s10439-015-1391-7

Cater, H. L., Sundstrom, L. E., \& Morrison, B. (2006). Temporal development of hippocampal cell death is dependent on tissue strain but not strain rate. Journal of Biomechanics, 39(15), 2810-2818. https://doi.org/10.1016/j.jbiomech.2005.09.023

Centers for Disease Control and Prevention. (2003). Report to Congress on mild traumatic brain injury in the United States: Steps to prevent a serious public health problem. Atlanta, GA: National Center for Injury Prevention and Control.

Centers for Disease Control and Prevention. (2015). Report to Congress on traumatic brain injury in the United States: Epidemiology and rehabilitation. Atlanta, GA: National Center for Injury Prevention and Control.

Chamouard, F., Trosseille, X., Pincemaille, Y., \& Tarriere, C. (1987). Methodological Aspects of an Experimental Research on Cerebral Tolerance on the Basis of Boxers' Training Fights. SAE Technical Paper.

Chatelin, S., Constantinesco, A., \& Willinger, R. (2010). Fifty years of brain tissue mechanical testing: From in vitro to in vivo investigations. Biorheology, (5-6), 255-276. https://doi.org/10.3233/BIR-2010-0576

Chiang Colvin, A., Mullen, J., Lovell, M. R., Vereeke West, R., Collins, M. W., \& Groh, M. (2009). The role of concussion history and gender in recovery from soccer-related concussion. The American Journal of Sports Medicine, 37(9), 1699-1704.

Cobb, B. R., Urban, J. E., Davenport, E. M., Rowson, S., Duma, S. M., Maldjian, J. A., ... Stitzel, J. D. (2013). Head Impact Exposure in Youth Football: Elementary School Ages 9-12 Years and the Effect of Practice Structure. Annals of Biomedical Engineering, 4l(12), 2463-2473. https://doi.org/10.1007/s10439-013-0867-6

Comm, S. T. I. S. (2003). Instrumentation for impact test-part 1-electronic instrumentation (j211/1). Warrendale, PA: Society of Automotive Engineers.

Coronado, V. G., Haileyesus, T., Cheng, T. A., Bell, J. M., Haarbauer-Krupa, J., Lionbarger, M. R., ... Gilchrist, J. (2015). Trends in Sports- and Recreation-Related Traumatic Brain Injuries Treated in US Emergency Departments: The National Electronic Injury Surveillance System-All Injury Program (NEISS-AIP) 2001-2012. Journal of Head Trauma Rehabilitation, 30(3), 185-197. https://doi.org/10.1097/HTR.0000000000000156

Covassin, T., \& Elbin, R. J. (2011). The Female Athlete: The Role of Gender in the Assessment and Management of Sport-Related Concussion. Clinics in Sports Medicine, 30(1), 125131. https://doi.org/10.1016/j.csm.2010.08.001

Crandall, J. R., Bose, D., Forman, J., Untaroiu, C. D., Arregui-Dalmases, C., Shaw, C. G., \& Kerrigan, J. R. (2011). Human surrogates for injury biomechanics research. Clinical Anatomy, 24(3), 362-371. https://doi.org/10.1002/ca.21152 
Crisco, J. J., Wilcox, B. J., Beckwith, J. G., Chu, J. J., Duhaime, A.-C., Rowson, S., ... Greenwald, R. M. (2011). Head impact exposure in collegiate football players. Journal of Biomechanics, 44(15), 2673-2678. https://doi.org/10.1016/j.jbiomech.2011.08.003

Cummiskey, B. R. (2015). Characterization and evaluation of head impact sensors and varsity football helmets. Purdue University.

Daniel, R. W., Rowson, S., \& Duma, S. M. (2012). Head Impact Exposure in Youth Football. Annals of Biomedical Engineering, 40(4), 976-981. https://doi.org/10.1007/s10439-0120530-7

Davis-Hayes, C., Gossett, J. D., Levine, W. N., Shams, T., Harada, J., Mitnick, J., \& Noble, J. (2017). Sex-specific Outcomes and Predictors of Concussion Recovery: Journal of the American Academy of Orthopaedic Surgeons, 25(12), 818-828. https://doi.org/10.5435/JAAOS-D-17-00276

De Courten-Myers, G. M. (1999). The human cerebral cortex: gender differences in structure and function. Journal of Neuropathology and Experimental Neurology, 58(3), 217-226.

Deck, C., \& Willinger, R. (2008). Improved head injury criteria based on head FE model. International Journal of Crashworthiness, 13(6), 667-678. https://doi.org/10.1080/13588260802411523

Declaration of Helsinki (1996).

Dick, R. W. (2009). Is there a gender difference in concussion incidence and outcomes? British Journal of Sports Medicine, 43(Suppl_1), i46-i50. https://doi.org/10.1136/bjsm.2009.058172

DiDomenico, L., \& Nusholtz, G. (2003). Comparison of Parametric and Non-Parametric Methods for Determining Injury Risk. https://doi.org/10.4271/2003-01-1362

DiMasi, F. (1995). Transformation of nine-accelerometer-package (NAP) data for replicating headpart kinematics and dynamic loading.

Dokko, Y., Anderson, R., Manavis, J., Blumburgs, P., McLean, J., Zhang, L., ... King, A. I. (2003). Validation of the human head FE model against pedestrian accident and its tentative application to the examination of the existing tolerance curve. In Proceedings 18th International Technical Conference on the Enhanced Safety Vehicle, Paper.

Doorly, M. C., \& Gilchrist, M. D. (2006). The use of accident reconstruction for the analysis of traumatic brain injury due to head impacts arising from falls. Computer Methods in Biomechanics and Biomedical Engineering, 9(6), 371-377. https://doi.org/10.1080/10255840601003551

Doorly, M. C., \& Gilchrist, M. D. (2009). Three-dimensional multibody dynamics analysis of accidental falls resulting in traumatic brain injury. International Journal of Crashworthiness, 14(5), 503-509. https://doi.org/10.1080/13588260902826554 
Doorly, M. C., Horgan, T. J., \& Gilchrist, M. D. (2005a). Reconstruction of head injury cases arising from falls using the UCD brain trauma model. In IUTAM Symposium on Impact Biomechanics: From Fundamental Insights to Applications (pp. 431-440). Springer.

Doorly, M. C., Phillips, J. P., \& Gilchrist, M. D. (2005b). Reconstructing Real Life Accidents Towards Establishing Criteria for Traumatic Head Impact Injuries. In M. D. Gilchrist (Ed.), IUTAM Symposium on Impact Biomechanics: From Fundamental Insights to Applications (Vol. 124, pp. 81-90). Berlin/Heidelberg: Springer-Verlag. https://doi.org/10.1007/1-4020-3796-1_8

Duma, S. M., Manoogian, S. J., Bussone, W. R., Brolinson, P. G., Goforth, M. W., Donnenwerth, J. J., ... Crisco, J. J. (2005). Analysis of real-time head accelerations in collegiate football players. Clinical Journal of Sport Medicine, 15(1), 3-8.

Eckner, J. T., Sabin, M., Kutcher, J. S., \& Broglio, S. P. (2011). No Evidence for a Cumulative Impact Effect on Concussion Injury Threshold. Journal of Neurotrauma, 28(10), 20792090. https://doi.org/10.1089/neu.2011.1910

Egan, J. P. (1975). Signal detection theory and $\{$ ROC $\}$ analysis.

Eppinger, R., Sun, E., Bandak, F., Haffner, M., Khaewpong, N., Maltese, M., ... others. (1999). Development of improved injury criteria for the assessment of advanced automotive restraint systems-II. National Highway Traffic Safety Administration, 1-70.

Evans, L. (2004). Traffic safety. Bloomfield, Mich: Science Serving Society.

Ewing, C. L., \& Thomas, D. J. (1972). Human Head and Neck Response to Impact Acceleration (No. NAMRL-Monograph-21). Pensacola, FL: Naval Aerospace Medical Research Lab.

Ewing, C. L., Thomas, D. J., Lustick, L., Becker, E., Willems, G., \& Muzzy, W. H. (1975). The Effect of the Initial Position of the Head and Neck on the Dynamic Response of the Human Head and Neck to -Gx Impact Acceleration (pp. 487-512). https://doi.org/10.4271/751157

Ewing, C. L., Thomas, D. J., Lustick, L., Muzzy, W. H., Willems, G. C., \& Majewski, P. (1978). Effect of initial position on the human head and neck response to $+Y$ impact acceleration. SAE Technical Paper.

Ewing, C. L., Thomas, D. J., Lustick, L., Muzzy, W. H., Willems, G., \& Majewski, P. L. (1976). The Effect of Duration, Rate of Onset, and Peak Sled Acceleration on the Dynamic Response of the Human Head and Neck. https://doi.org/10.4271/760800

Ewing, C. L., Thomas, D. J., Lustik, L., Muzzy, W. H., Willems, G. C., \& Majewski, P. (1977). Dynamic Response of the Human Head and Neck to +Gy Impact Acceleration. Stapp Car Crash Conference. https://doi.org/10.4271/770928

Ewing, C. L., Thomas, D. J., Patrick, L. M., Beeler, G. W., \& Smith, M. J. (1969). Living Human Dynamic Response to - Gx Impact Acceleration II-Accelerations Measured on the Head and Neck. SAE Technical Paper. 
Faraggi, D., \& Reiser, B. (2002). Estimation of the area under the ROC curve. Statistics in Medicine, 21(20), 3093-3106. https://doi.org/10.1002/sim.1228

Farhat, M. Y., Abi-Younes, S., \& Ramwell, P. W. (1996). Non-genomic effects of estrogen and the vessel wall. Biochemical Pharmacology, 51(5), 571-576.

Fréchède, B., \& McIntosh, A. (2007). Use of MADYMO's human facet model to evaluate the risk of head injury in impact. In 20th International Technical Conference on the Enhanced Safety of Vehicles (pp. 1-14).

Fréchède, B., \& McIntosh, A. S. (2009). Numerical Reconstruction of Real-Life Concussive Football Impacts: Medicine \& Science in Sports \& Exercise, 41(2), 390-398. https://doi.org/10.1249/MSS.0b013e318186b1c5

Funk, J. R., Cormier, J. M., Bain, C. E., Guzman, H., Bonugli, E., \& Manoogian, S. J. (2011). Head and Neck Loading in Everyday and Vigorous Activities. Annals of Biomedical Engineering, 39(2), 766-776. https://doi.org/10.1007/s10439-010-0183-3

Funk, J. R., Duma, S. M., Manoogian, S. J., \& Rowson, S. (2007). Biomechanical risk estimates for mild traumatic brain injury. Annual Proceedings. Association for the Advancement of Automotive Medicine, 51, 343-361.

Funk, J. R., Rowson, S., Daniel, R. W., \& Duma, S. M. (2012). Validation of concussion risk curves for collegiate football players derived from HITS data. Annals of Biomedical Engineering, 40(1), 79-89.

Gabler, L. F., Crandall, J. R., \& Panzer, M. B. (2016a). Assessment of kinematic brain injury metrics for predicting strain responses in diverse automotive impact conditions. Annals of Biomedical Engineering (in Review).

Gabler, L. F., Crandall, J. R., \& Panzer, M. B. (2016b). Investigating Brain Injury Tolerance in the Sagittal Plane Using a Finite Element Model of the Human Head. International Journal of Automotive Engineering, 7(1), 37-43.

Gadd, C. W. (1962). Criteria for injury potential. Impact Acceleration Stress, 977, 141.

Gennarelli, T. A. (2005). Neuropathology. In J. M. Silver, T. W. McAllister, \& S. C. Yudofsky, Textbook of Traumatic Brain Injury (pp. 27-34). Arlington, VA: American Psychiatric Publishing, Inc.

Gennarelli, T. A., Thibault, L. E., Adams, J. H., Graham, D. I., Thompson, C. J., \& Marcincin, R. P. (1982). Diffuse axonal injury and traumatic coma in the primate. Annals of Neurology, 12(6), 564-574.

Gennarelli, T. A., \& Wodzin, E. (2008). Abbreviated injury scale 2005: update 2008. Russ Reeder.

Ghajari, M., Hellyer, P. J., \& Sharp, D. J. (2017). Computational modelling of traumatic brain injury predicts the location of chronic traumatic encephalopathy pathology. Brain, 140(2), 333-343. https://doi.org/10.1093/brain/aww317 
Gilchrist, J., Thomas, K. E., Xu, L., McGuire, L., \& Coronado, V. (2011). Nonfatal traumatic brain injuries related to sports and recreation activities among persons aged $\leq 19$ YearsUnited States, 2001-2009. Morbidity and Mortality Weekly Report, 60(39), 1337-1342.

Gilchrist, M. D., \& Doorly, M. C. (2009). An in-depth analysis of real world fall accidents involving brain trauma. In A. Gefen (Ed.), The Pathomechanics of Tissue Injury and Disease, and the Mechanophysiology of Healing. Research Signpost. Retrieved from http://researchrepository.ucd.ie/handle/10197/5897

Giordano, C., \& Kleiven, S. (2014). Evaluation of axonal strain as a predictor for mild traumatic brain injuries using finite element modeling. Stapp Car Crash Journal, 58, 29.

Greenwald, R. M., Gwin, J. T., Chu, J. J., \& Crisco, J. J. (2008). HEAD IMPACT SEVERITY MEASURES FOR EVALUATING MILD TRAUMATIC BRAIN INJURY RISK EXPOSURE. Neurosurgery, 62(4), 789-798. https://doi.org/10.1227/01.neu.0000318162.67472.ad

Gurdjian, E. S., Roberts, V. L., \& Thomas, L. M. (1966). Tolerance curves of acceleration and intracranial pressure and protective index in experimental head injury. Journal of Trauma and Acute Care Surgery, 6(5), 600-604.

Guskiewicz, K. M., Mihalik, J. P., Shankar, V., Marshall, S. W., Crowell, D. H., Oliaro, S. M., ... Hooker, D. N. (2007). Measurement of Head Impacts in Collegiate Football Players. Neurosurgery, 61(6), 1244-1253. https://doi.org/10.1227/01.neu.0000306103.68635.1a

Gysland, S. M., Mihalik, J. P., Register-Mihalik, J. K., Trulock, S. C., Shields, E. W., \& Guskiewicz, K. M. (2012). The Relationship Between Subconcussive Impacts and Concussion History on Clinical Measures of Neurologic Function in Collegiate Football Players. Annals of Biomedical Engineering, 40(1), 14-22. https://doi.org/10.1007/s10439-011-0421-3

Hanley, J. A., \& McNeil, B. J. (1982). The meaning and use of the area under a receiver operating characteristic (ROC) curve. Radiology, 143(1), 29-36. https://doi.org/10.1148/radiology.143.1.7063747

Hanley, J. A., \& McNeil, B. J. (1983). A method of comparing the areas under receiver operating characteristic curves derived from the same cases. Radiology, 148(3), 839-843. https://doi.org/10.1148/radiology.148.3.6878708

Harmon, K. G., Drezner, J. A., Gammons, M., Guskiewicz, K. M., Halstead, M., Herring, S. A., ... Roberts, W. O. (2013). American Medical Society for Sports Medicine position statement: concussion in sport. British Journal of Sports Medicine, 47(1), 15-26. https://doi.org/10.1136/bjsports-2012-091941

Harper, B., Siyufy, A., Castleberry, J., Mickle, A., Jagger, K., Waff, A., \& Cox, K. (2015). Clinical Experiences Using a Hit Impact Indicator in Youth Football. The Sport Journal. Retrieved from http://thesportjournal.org/article/clinical-experiences-using-a-hit-impactindicator-in-youth-football/ 
Hendler, E., O’Rourke, J., Schulman, M., Katzeff, M., Domzalski, L., \& Rodgers, S. (1974). Effect of Head and Body Position and Muscular Tensing on Response to Impact. Crew Systems Dept. Naval Air Development Center, 18(741184).

Hernandez, F., Wu, L. C., Yip, M. C., Hoffman, A. R., Lopez, J., Grant, G., ... Camarillo, D. B. (2015). Six Degree of Freedom Measurements of Human Mild Traumatic Brain Injury. Annals of Biomedical Engineering, 43(8), 1918-1934. https://doi.org/10.1007/s10439014-1212-4

Holbourn, A. H. S. (1943). Mechanics of head injuries. The Lancet, 242(6267), 438-441.

Hootman, J. M., Dick, R., \& Agel, J. (2007). Epidemiology of Collegiate Injuries for 15 Sports: Summary and Recommendations for Injury Prevention Initiatives. Journal of Athletic Training, 42(2), 311-319.

Jadischke, R., Viano, D. C., Dau, N., King, A. I., \& McCarthy, J. (2013). On the accuracy of the Head Impact Telemetry (HIT) System used in football helmets. Journal of Biomechanics, 46(13), 2310-2315.

Ji, S., Ghadyani, H., Bolander, R. P., Beckwith, J. G., Ford, J. C., McAllister, T. W., ... Jain, S. (2014). Parametric comparisons of intracranial mechanical responses from three validated finite element models of the human head. Annals of Biomedical Engineering, 42(1), 1124.

Kahane, C. J. (2015). Lives saved by vehicle safety technologies and associated Federal Motor Vehicle Safety Standards, 1960 to 2012-Passenger cars and LTVs-With reviews of 26 FMVSS and the effectiveness of their associated safety technologies in reducing fatalities, injuries, and crashes. Report No. DOT HS, 812, 069.

Kang, H.-S., Willinger, R., Diaw, B. M., \& Chinn, B. (1997). Validation of a 3D anatomic human head model and replication of head impact in motorcycle accident by finite element modeling. In Proceedings: Stapp Car Crash Conference (Vol. 41, pp. 329-338). Society of Automotive Engineers SAE.

Kannel, W. B., \& Thom, T. J. (1994). Incidence, prevalence, and mortality of cardiovascular diseases. The Heart: Arteries and Veins, 185-201.

Kendall, M., Post, A., \& Gilchrist, M. D. (2012). A comparison of dynamic impact response and brain deformation metrics within the cerebrum of head impact reconstructions representing three mechanisms of head injury in ice hockey. In IRCOBI Conference 2012. International Research Council on the Biomechanics of Injury.

Kent, R. W., \& Bass, C. R. (2007). Experimentación con animales (Experimentation with animals). In C. A. Dalmases, E. D. G. de Tráfico, J. L. Narro, \& M. Seguí-Gómez, Fundamentos de biomecánica en las lesiones por accidentes de tráfico. Dirección General de Tráfico. 
Kent, R. W., \& Funk, J. R. (2004). Data censoring and parametric distribution assignment in the development of injury risk functions from biochemical data. SAE Technical Paper. Retrieved from http://papers.sae.org/2004-01-0317/

Kerr, Z. Y., Yeargin, S. W., Valovich McLeod, T. C., Mensch, J., Hayden, R., \& Dompier, T. P. (2015). Comprehensive Coach Education Reduces Head Impact Exposure in American Youth Football. Orthopaedic Journal of Sports Medicine, 3(10), 232596711561054. https://doi.org/10.1177/2325967115610545

Kimpara, H., \& Iwamoto, M. (2012). Mild traumatic brain injury predictors based on angular accelerations during impacts. Annals of Biomedical Engineering, 40(1), 114-126.

Kimpara, H., Nakahira, Y., Iwamoto, M., Rowson, S., \& Duma, S. (2011). Head injury prediction methods based on 6 degree of freedom head acceleration measurements during impact. International Journal of Automotive Engineering, 2(2), 13-19.

King, A. I., Yang, K. H., Zhang, L., Hardy, W., \& Viano, D. C. (2003). Is head injury caused by linear or angular acceleration. In Proceedings of the International Research Conference on the Biomechanics of Impacts (IRCOBI) (pp. 1-12).

King, D. A., Hume, P. A., Brughelli, M., \& Gissane, C. (2015). Instrumented Mouthguard Acceleration Analyses for Head Impacts in Amateur Rugby Union Players Over a Season of Matches. The American Journal of Sports Medicine, 43(3), 614-624. https://doi.org/10.1177/0363546514560876

King, D. A., Hume, P. A., Gissane, C., \& Clark, T. N. (2016). Similar head impact acceleration measured using instrumented ear patches in a junior rugby union team during matches in comparison with other sports. Journal of Neurosurgery: Pediatrics, 18(1), 65-72. https://doi.org/10.3171/2015.12.PEDS15605

Kleiven, S. (2006). Evaluation of head injury criteria using a finite element model validated against experiments on localized brain motion, intracerebral acceleration, and intracranial pressure. International Journal of Crashworthiness, 11(1), 65-79. https://doi.org/10.1533/ijcr.2005.0384

Kleiven, S. (2007). Predictors for traumatic brain injuries evaluated through accident reconstructions. Stapp Car Crash J, 51, 81-114.

Knox, T. (2004). Validation of Earplug Accelerometers as a Means of Measuring Head Motion. https://doi.org/10.4271/2004-01-3538

Knox, T., Pellettiere, J., Perry, C., Plaga, J., \& Bonfeld, J. (2009). New sensors to track head acceleration during possible injurious events. ENDEVCO CORP SAN JUAN CAPISTRANO CA.

Kucera, K. L., Klossner, D., Colgate, B., \& Cantu, R. C. (2014). Annual Survey of Football Injury Research, 1931-2014. National Center for Catastrophic Injury Research. Http://Nccsir. Unc. Edu/Reports/(Accessed 02 Aug 2015). 
Kumar, S., Ferrari, R., Narayan, Y., \& Jones, T. (2006). The effect of a 3-point harness restraint and car seat in whiplash-type lateral impacts. Spine, 31(1), E11-18.

Kuo, C., Wu, L. C., Hammoor, B. T., Luck, J. F., Cutcliffe, H. C., Lynall, R. C., ... Camarillo, D. B. (2016). Effect of the mandible on mouthguard measurements of head kinematics. Journal of Biomechanics, 49(9), 1845-1853. https://doi.org/10.1016/j.jbiomech.2016.04.017

Laituri, T. R., El-Jawahri, R. E., Henry, S., \& Sullivan, K. (2015). Field-based Assessments of Various AIS2+ Head Risk Curves for Frontal Impact. SAE Technical Paper.

LaPlaca, M. C., Simon, C. M., Prado, G. R., \& Cullen, D. K. (2007). CNS injury biomechanics and experimental models. In Progress in Brain Research (Vol. 161, pp. 13-26). Elsevier. https://doi.org/10.1016/S0079-6123(06)61002-9

Lewis, L. M., Naunheim, R., Standeven, J., Lauryssen, C., Richter, C., \& Jeffords, B. (2001). Do Football Helmets Reduce Acceleration of Impact in Blunt Head Injuries? Academic Emergency Medicine, 8(6), 604-609. https://doi.org/10.1111/j.1553-2712.2001.tb00171.x

Lincoln, A. E., Caswell, S. V., Almquist, J. L., Dunn, R. E., Norris, J. B., \& Hinton, R. Y. (2011). Trends in Concussion Incidence in High School Sports: A Prospective 11-Year Study. The American Journal of Sports Medicine, 39(5), 958-963. https://doi.org/10.1177/0363546510392326

Lissner, H. R., Lebow, M., \& Evans, F. G. (1960). Experimental studies on the relation between acceleration and intracranial pressure changes in man. Surgery, Gynecology \& Obstetrics, $111,329-338$.

Lucchetti, L., Cappozzo, A., Cappello, A., \& Croce, U. D. (1998). Skin movement artefact assessment and compensation in the estimation of knee-joint kinematics. Journal of Biomechanics, 31(11), 977-984. https://doi.org/10.1016/S0021-9290(98)00083-9

Lynall, R. C., Clark, M. D., Grand, E. E., Stucker, J. C., Littleton, A. C., Aguilar, A. J., ... Mihalik, J. P. (2016). Head Impact Biomechanics in Women's College Soccer: Medicine \& Science in Sports \& Exercise, 48(9), 1772-1778. https://doi.org/10.1249/MSS.0000000000000951

Manoogian, S. J., McNeely, D., Duma, S. M., Brolinson, G., \& Greenwald, R. (2006). Head acceleration is less than 10 percent of helmet acceleration in football impacts. Biomedical Sciences Instrumentation, 42, 383-388.

Mao, H., Zhang, L., Jiang, B., Genthikatti, V. V., Jin, X., Zhu, F., .. Singh, A. (2013). Development of a finite element human head model partially validated with thirty five experimental cases. Journal of Biomechanical Engineering, 135(11), 111002.

Margulies, S. S., Meaney, D. F., Smith, D., Chen, X. H., Miller, R., \& Raghupathi, R. (1999). A comparison of diffuse brain injury in the newborn and adult pig. In Proceedings of the International Research Council on the Biomechanics of Injury conference (Vol. 27, pp. 133-145). International Research Council on Biomechanics of Injury. 
Margulies, S. S., \& Thibault, L. E. (1992). A proposed tolerance criterion for diffuse axonal injury in man. Journal of Biomechanics, 25(8), 917-923.

Marjoux, D., Baumgartner, D., Deck, C., \& Willinger, R. (2008). Head injury prediction capability of the HIC, HIP, SIMon and ULP criteria. Accident Analysis \& Prevention, 40(3), 1135-1148. https://doi.org/10.1016/j.aap.2007.12.006

Marr, A. L., \& Coronado, V. G. (2004). Central nervous system injury surveillance data submission standards-2002. Atlanta, GA: Centers for Disease Control and Prevention, National Center for Injury Prevention and Control.

McCaffrey, M. A., Mihalik, J. P., Crowell, D. H., Shields, E. W., \& Guskiewicz, K. M. (2007). Measurement of head impacts in collegiate football players: Clinical measures of concussion after high- and low-magnitude impacts. Neurosurgery, 61(6), 1236-1243. https://doi.org/10.1227/01.neu.0000306102.91506.8b

McCrory, P., Meeuwisse, W. H., Aubry, M., Cantu, B., Dvořák, J., Echemendia, R. J., ... Turner, M. (2013). Consensus statement on concussion in sport: the 4th International Conference on Concussion in Sport held in Zurich, November 2012. British Journal of Sports Medicine, 47(5), 250-258. https://doi.org/10.1136/bjsports-2013-092313

McCuen, E., Svaldi, D., Breedlove, K., Kraz, N., Cummiskey, B., Breedlove, E. L., ... Nauman, E. A. (2015). Collegiate women's soccer players suffer greater cumulative head impacts than their high school counterparts. Journal of Biomechanics, 48(13), 3720-3723. https://doi.org/10.1016/j.jbiomech.2015.08.003

McIntosh, A. S., McCrory, P., \& Comerford, J. (2000). The dynamics of concussive head impacts in rugby and Australian rules football: Medicine and Science in Sports and Exercise, 32(12), 1980-1984. https://doi.org/10.1097/00005768-200012000-00002

McIntosh, A. S., Patton, D. A., Fréchède, B., Pierré, P.-A., Ferry, E., \& Barthels, T. (2014). The biomechanics of concussion in unhelmeted football players in Australia: a case-control study. BMJ Open, 4(5), e005078. https://doi.org/10.1136/bmjopen-2014-005078

Meaney, D. F., Smith, D., Ross, D. T., \& Gennarelli, T. A. (1993). Diffuse axonal injury in the miniature pig: biomechanical development and injury threshold. ASME APPLIED MECHANICS DIVISION-PUBLICATIONS-AMD, 169, 169-169.

Mihalik, J. P., Bell, D. R., Marshall, S. W., \& Guskiewicz, K. M. (2007). Measurement of Head Impacts in Collegiate Football Players: An Investigation of Positional and Event-type Differences. Neurosurgery, 61(6), 1229-1235. https://doi.org/10.1227/01.neu.0000306101.83882.c8

Mihalik, J. P., Blackburn, J. T., Greenwald, R. M., Cantu, R. C., Marshall, S. W., \& Guskiewicz, K. M. (2010a). Collision Type and Player Anticipation Affect Head Impact Severity Among Youth Ice Hockey Players. PEDIATRICS, 125(6), e1394-e1401. https://doi.org/10.1542/peds.2009-2849 
Mihalik, J. P., Greenwald, R. M., Blackburn, J. T., Cantu, R. C., Marshall, S. W., \& Guskiewicz, K. M. (2010b). Effect of Infraction Type on Head Impact Severity in Youth Ice Hockey: Medicine \& Science in Sports \& Exercise, 42(8), 1431-1438. https://doi.org/10.1249/MSS.0b013e3181d2521a

Mihalik, J. P., Guskiewicz, K. M., Jeffries, J. A., Greenwald, R. M., \& Marshall, S. W. (2008). Characteristics of head impacts sustained by youth ice hockey players. Proceedings of the Institution of Mechanical Engineers, Part P: Journal of Sports Engineering and Technology, 222(1), 45-52. https://doi.org/10.1243/17543371JSET4

Mihalik, J. P., Guskiewicz, K. M., Marshall, S. W., Blackburn, J. T., Cantu, R. C., \& Greenwald, R. M. (2012). Head Impact Biomechanics in Youth Hockey: Comparisons Across Playing Position, Event Types, and Impact Locations. Annals of Biomedical Engineering, 40(1), 141-149. https://doi.org/10.1007/s10439-011-0405-3

Miyashita, T., Diakogeorgiou, E., Marrie, K., \& Danaher, R. (2016). Frequency and Location of Head Impacts in Division I Men's Lacrosse Players. Athletic Training \& Sports Health Care, 8(5), 202-208. https://doi.org/10.3928/19425864-20160503-01

Morrison, B., Cater, H. L., Wang, C. C., \& Thomas, F. C. (2003). A tissue level tolerance criterion for living brain developed with an in vitro model of traumatic mechanical loading. Stapp Car Crash Journal, 47, 93.

Mueller, B., MacAlister, A., Nolan, J., \& Zuby, D. (2015). Comparison of HIC and BRIC Head Injury Risk in IIHS Frontal Crash Tests to Real-World Head Injuries. In 24th International Technical Conference on the Enhanced Safety of Vehicles (ESV).

Nahum, A. M., Smith, R., \& Ward, C. C. (1977). Intracranial pressure dynamics during head impact. SAE Technical Paper.

Naunheim, R. S., Standeven, J., Richter, C., \& Lewis, L. M. (2000). Comparison of Impact Data in Hockey, Football, and Soccer. The Journal of Trauma: Injury, Infection, and Critical Care.

Newman, J. (1986). A generalized acceleration model for brain injury threshold (GAMBIT). In Proc. IRCOBI Conf (pp. 121-131).

Newman, J., Beusenberg, M., Fournier, E., Shewchenko, N., Withnall, C., King, A., ... Thibault, L. (1999). A new biomechanical assessment of mild traumatic brain injury. Part 1 Methodology. In Proceedings of the International Research Conference on the Biomechanics of Impacts (IRCOBI) (pp. 17-36). Sitges, Spain: International Research Council on Biomechanics of Injury.

Newman, J., Shewchenko, N., \& Welbourne, E. (2000a). A proposed new biomechanical head injury assessment function-the maximum power index. Stapp Car Crash Journal, 44, 215-247.

Newman, J., Barr, C., Beusenberg, M. C., Fournier, E., Shewchenko, N., Welbourne, E., \& Withnall, C. (2000b). A new biomechanical assessment of mild traumatic brain injury. 
Part 2: results and conclusions. In Proceedings of the International Research Council on the Biomechanics of Injury conference (Vol. 28). International Research Council on Biomechanics of Injury.

Newman, J., Beusenberg, M., Shewchenko, N., Withnall, C., \& Fournier, E. (2005). Verification of biomechanical methods employed in a comprehensive study of mild traumatic brain injury and the effectiveness of American football helmets. Journal of Biomechanics, 38(7), 1469-1481. https://doi.org/10.1016/j.jbiomech.2004.06.025

Ng, T. P., Bussone, W. R., \& Duma, S. M. (2006). The effect of gender and body size on linear accelerations of the head observed during daily activities. Biomedical Sciences Instrumentation, 42, 25-30.

NHTSA. (1995). Final economic assessment, FMVSS No. 201, upper interior head protection (NHTSA docket 1996-1762-0003). Office of Regulatory Analysis.

NHTSA. (2000). Title 49 Code of Federal Regulations (CFR) Part 571 Section 208, Occupant Crash Protection. Washington, DC: Office of the Federal Register, National Archives and Records Administration.

NHTSA. New Car Assessment Program (2015).

NHTSA VSR | Query Biomechanicss Test Database on select test parameters. (n.d.). Retrieved December 21, 2015, from https://wwwnrd.nhtsa.dot.gov/database/VSR/bio/QueryTest.aspx

NOCSAE. (2015). Standard Performance Specification for Newly Manufactured Football Helmets. Retrieved from http://nocsae.org/wpcontent/files_mf/1436291444ND00213m15MfrdFBHelmetsStandardPerformance.pdf

Oeur, R. A., Karton, C., Post, A., Rousseau, P., Hoshizaki, T. B., Marshall, S., .. Gilchrist, M. D. (2015). A comparison of head dynamic response and brain tissue stress and strain using accident reconstructions for concussion, concussion with persistent postconcussive symptoms, and subdural hematoma. Journal of Neurosurgery, 123(2), 415-422. https://doi.org/10.3171/2014.10.JNS14440

Olafsdottir, J. M., Östh, J., Davidsson, J., \& Brolin, K. (2013). Passenger kinematics and muscle responses in autonomous braking events with standard and reversible pre-tensioned restraints. In IRCOBI Conference 2013 (pp. 602-617).

Ommaya, A. K. (1985). Biomechanics of head injury: experimental aspects. The Biomechanics of Trauma, 13, 245-269.

Ommaya, A. K., \& Gennarelli, T. A. (1974). Cerebral concussion and traumatic unconsciousness. Brain, 97(1), 633-654.

Ommaya, A. K., Rockoff, S. D., Baldwin, M., \& Friauf, W. S. (1964). Experimental Concussion: A First Report. Journal of Neurosurgery, 21(4), 249-265. https://doi.org/10.3171/jns.1964.21.4.0249 
O’Riordain, K., Thomas, P. M., Phillips, J. P., \& Gilchrist, M. D. (2003). Reconstruction of real world head injury accidents resulting from falls using multibody dynamics. Clinical Biomechanics, 18(7), 590-600. https://doi.org/10.1016/S0268-0033(03)00111-6

O’Sullivan, D. M., \& Fife, G. P. (2017). Biomechanical head impact characteristics during sparring practice sessions in high school taekwondo athletes. Journal of Neurosurgery: Pediatrics, 19(6), 662-667. https://doi.org/10.3171/2017.1.PEDS16432

Padgaonkar, A. J., Krieger, K. W., \& King, A. I. (1975). Measurement of Angular Acceleration of a Rigid Body Using Linear Accelerometers. Journal of Applied Mechanics, 42(3), 552-556. https://doi.org/10.1115/1.3423640

Panzer, M. B., Myers, B. S., Capehart, B. P., \& Bass, C. R. (2012). Development of a finite element model for blast brain injury and the effects of CSF cavitation. Annals of Biomedical Engineering, 40(7), 1530-1544.

Panzer, M. B., Wood, G. W., \& Bass, C. R. (2014). Scaling in neurotrauma: how do we apply animal experiments to people? Experimental Neurology, 261, 120-126.

Pascrell, C. B. (2009). Introduction to the Report of the International Conference on Behavioral Health and Traumatic Brain Injury. The Clinical Neuropsychologist, 23(8), 1281-1290. https://doi.org/10.1080/13854040902939319

Patrick, L. M., Lissner, H. R., \& Gurdjian, E. S. (1963). Survival by design: Head protection. In Proceedings: American Association for Automotive Medicine Annual Conference (Vol. 7, pp. 483-499). Association for the Advancement of Automotive Medicine.

Patton, D. A. (2016). A Review of Instrumented Equipment to Investigate Head Impacts in Sport. Applied Bionics and Biomechanics, 2016, 1-16. https://doi.org/10.1155/2016/7049743

Pellman, E. J., Powell, J. W., Viano, D. C., Casson, I. R., Tucker, A. M., Feuer, H., ... Robertson, D. W. (2004a). Concussion in Professional Football: Epidemiological Features of Game Injuries and Review of the Literature-Part 3. Neurosurgery, 54(1), 81-96. https://doi.org/10.1227/01.NEU.0000097267.54786.54

Pellman, E. J., Viano, D. C., Casson, I. R., Arfken, C., \& Feuer, H. (2005). Concussion in Professional Football: Players Returning to the Same Game-Part 7: Neurosurgery, 56(1), 79-92. https://doi.org/10.1227/01.NEU.0000150180.16552.8D

Pellman, E. J., Viano, D. C., Casson, I. R., Arfken, C., \& Powell, J. (2004b). Concussion in Professional Football: Injuries Involving 7 or More Days Out-Part 5. Neurosurgery, 55(5), 1100-1119. https://doi.org/10.1227/01.NEU.0000147063.12873.F5

Pellman, E. J., Viano, D. C., Tucker, A. M., Casson, I. R., \& Waeckerle, J. F. (2003). Concussion in professional football: reconstruction of game impacts and injuries. Neurosurgery, 53(4), 799-814. 
Pincemaille, Y., Trosselle, X., Mack, P., Tarriere, C., Breton, F., \& Renault, B. (1989). Some New Data Related to Human Tolerance Obtained from Volunteer Boxers, 33(892435).

Post, A. (2013, July 22). The Influence of Dynamic Response Characteristics on Traumatic Brain Injury. University of Ottawa, Ottawa, Canada. https://doi.org/10.20381/ruor-3099

Post, A., Hoshizaki, T. B., Gilchrist, M. D., Koncan, D., Dawson, L., Chen, W., ... The Pediatric Emergency Research Canada (PERC) 5P Concussion Team. (2017). A comparison in a youth population between those with and without a history of concussion using biomechanical reconstruction. Journal of Neurosurgery: Pediatrics, 19(4), 502-510. https://doi.org/10.3171/2016.10.PEDS16449

Post, A., Kendall, M., Koncan, D., Cournoyer, J., Blaine Hoshizaki, T., Gilchrist, M. D., ... Marshall, S. (2015). Characterization of persistent concussive syndrome using injury reconstruction and finite element modelling. Journal of the Mechanical Behavior of Biomedical Materials, 41, 325-335. https://doi.org/10.1016/j.jmbbm.2014.07.034

Prasad, P., Dalmotas, D., \& German, A. (2014). The field relevance of NHTSA's oblique research moving deformable barrier tests. Stapp Car Crash Journal, 58, 175.

Prasad, P., \& Mertz, H. J. (1985). The position of the United States delegation to the ISO working group on the use of HIC in the automotive environment. SAE Transactions, 94, $106-116$.

Prentice, R. L., \& Pyke, R. (1979). Logistic Disease Incidence Models and Case-Control Studies. Biometrika, 66(3), 403. https://doi.org/10.2307/2335158

Press, J. N., \& Rowson, S. (2017). Quantifying Head Impact Exposure in Collegiate Women's Soccer: Clinical Journal of Sport Medicine, 27(2), 104-110. https://doi.org/10.1097/JSM.0000000000000313

Raul, J.-S., Baumgartner, D., Willinger, R., \& Ludes, B. (2006). Finite element modelling of human head injuries caused by a fall. International Journal of Legal Medicine, 120(4), 212-218. https://doi.org/10.1007/s00414-005-0018-1

Reynolds, B. B., Patrie, J., Henry, E. J., Goodkin, H. P., Broshek, D. K., Wintermark, M., \& Druzgal, T. J. (2016). Quantifying Head Impacts in Collegiate Lacrosse. The American Journal of Sports Medicine, 44(11), 2947-2956. https://doi.org/10.1177/0363546516648442

Reynolds, B. B., Patrie, J., Henry, E. J., Goodkin, H. P., Broshek, D. K., Wintermark, M., \& Druzgal, T. J. (2017). Comparative Analysis of Head Impact in Contact and Collision Sports. Journal of Neurotrauma, 34(1), 38-49. https://doi.org/10.1089/neu.2015.4308

Roof, R. L., Duvdevani, R., \& Stein, D. G. (1993). Gender influences outcome of brain injury: progesterone plays a protective role. Brain Research, 607(1), 333-336.

Rosner, B. (2015). Fundamentals of biostatistics. Nelson Education. 
Rowson, B., Rowson, S., \& Duma, S. M. (2015). Hockey STAR: A Methodology for Assessing the Biomechanical Performance of Hockey Helmets. Annals of Biomedical Engineering, 43(10), 2429-2443. https://doi.org/10.1007/s10439-015-1278-7

Rowson, B., Rowson, S., \& Duma, S. M. (2017a). Assessing brain injury criteria through reconstructions of head impacts in football. Presented at the Biomedical Engineering Society Annual Meeting, Phoenix, AZ.

Rowson, B., Rowson, S., \& Duma, S. M. (2017b). Characterizing brain injury criteria for concussion through reconstructions of collegiate football head impacts. Presented at the Summer Biomechanics, Bioengineering and Biotransport Conference, Tucson, AZ.

Rowson, B., Rowson, S., \& Duma, S. M. (2017c). Evaluating the ability of head injury criteria to predict concussion through reconstructions of collegiate football head impacts. In JOURNAL OF NEUROTRAUMA (Vol. 34, pp. A43-A44). MARY ANN LIEBERT, INC 140 HUGUENOT STREET, 3RD FL, NEW ROCHELLE, NY 10801 USA.

Rowson, S., Brolinson, G., Goforth, M., Dietter, D., \& Duma, S. (2009). Linear and Angular Head Acceleration Measurements in Collegiate Football. Journal of Biomechanical Engineering, 131(6), 061016. https://doi.org/10.1115/1.3130454

Rowson, S., \& Duma, D., Stefan M. (2013). Brain injury prediction: Assessing the combined probability of concussion using linear and rotational head acceleration. Annals of Biomedical Engineering, 41, 873-882.

Rowson, S., \& Duma, S. M. (2011). Development of the STAR evaluation system for football helmets: integrating player head impact exposure and risk of concussion. Annals of Biomedical Engineering, 39(8), 2130-2140.

Rowson, S., Duma, S. M., Beckwith, J. G., Chu, J. J., Greenwald, R. M., Crisco, J. J., ... Maerlender, A. C. (2012). Rotational head kinematics in football impacts: An injury risk function for concussion. Annals of Biomedical Engineering, 40(1), 1-13. https://doi.org/10.1007/s10439-011-0392-4

Rumsey, D. J. (2016). Statistics For Dummies. John Wiley \& Sons.

Sanchez, E. J., Gabler, L. F., McGhee, J. S., Olszko, A. V., Chancey, V. C., Crandall, J. R., \& Panzer, M. B. (2017a). Evaluation of Head and Brain Injury Risk Functions Using SubInjurious Human Volunteer Data. Journal of Neurotrauma, 34(16), 2410-2424. https://doi.org/10.1089/neu.2016.4681

Sanchez, E. J., Gabler, L., Rowson, B., Rowson, S., Duma, S., \& Panzer, M. (2017b). Brain Strain Patterns Associated with Football Impact Reconstructions. In Journal of Neurotrauma (Vol. 34, pp. A30-A31). Mary Ann Liebert, Inc.

Schnebel, B., Gwin, J. T., Anderson, S., \& Gatlin, R. (2007). In Vivo Study of Head Impacts in Football: A Comparison of National Collegiate Athletic Association Division I Versus High School Impacts. Neurosurgery, 60(3), 490-496. https://doi.org/10.1227/01.NEU.0000249286.92255.7F 
Seeman, M. R., Muzzy, W. H., \& Lustick, L. S. (1986). Comparison of human and Hybrid III head and neck dynamic response. In Proceedings of the 30th Stapp Car Crash Conference.

Self, B. P., Karins, C., \& Knox, T. (2004). Head accelerations during impact events. In 5th International Conference on the Engineering of Sport. Davis, CA.

Shultz, R., Kedgley, A. E., \& Jenkyn, T. R. (2011). Quantifying skin motion artifact error of the hindfoot and forefoot marker clusters with the optical tracking of a multi-segment foot model using single-plane fluoroscopy. Gait \& Posture, 34(1), 44-48.

https://doi.org/10.1016/j.gaitpost.2011.03.008

Siegmund, G. P., Guskiewicz, K. M., Marshall, S. W., DeMarco, A. L., \& Bonin, S. J. (2014). A Headform for Testing Helmet and Mouthguard Sensors that Measure Head Impact Severity in Football Players. Annals of Biomedical Engineering, 42(9), 1834-1845. https://doi.org/10.1007/s10439-014-1052-2

Siegmund, G. P., Guskiewicz, K. M., Marshall, S. W., DeMarco, A. L., \& Bonin, S. J. (2015). Laboratory validation of two wearable sensor systems for measuring head impact severity in football players. Annals of Biomedical Engineering, 1-18.

Smith, D. H., \& Meaney, D. F. (2002). Roller Coasters, G Forces, and Brain Trauma: On the Wrong Track? Journal of Neurotrauma, 19(10), 1117-1120.

https://doi.org/10.1089/08977150260337921

Somers, J. T., Granderson, B., Melvin, J. W., Tabiei, A., Lawrence, C., Feiveson, A., ... Patalak, J. (2011). Development of head injury assessment reference values based on NASA injury modeling. Stapp Car Crash Journal, 55, 49.

Stalnaker, R. L., Alem, N. M., \& Benson, J. B. (1977). Validation studies for head impact injury model.

Stapp, J. P. (1951). Human Exposure to Linear Deceleration. Part 2: the Forward-facing Position and the Development of a Crash Harness.

Stapp, J. P., \& Taylor, E. R. (1964). Space cabin landing impact vector effects on human physiology. AIR FORCE RESEARCH LAB WRIGHT-PATTERSON AFB OH.

Stucker, J. C. (2015). Head impact biomechanics in collegiate female soccer players. The University of North Carolina at Chapel Hill.

Sullivan, S., Eucker, S. A., Gabrieli, D., Bradfield, C., Coats, B., Maltese, M. R., ... Margulies, S. S. (2015). White matter tract-oriented deformation predicts traumatic axonal brain injury and reveals rotational direction-specific vulnerabilities. Biomechanics and Modeling in Mechanobiology, 14(4), 877-896. https://doi.org/10.1007/s10237-014-0643$\mathrm{Z}$

Swartz, E. E., Broglio, S. P., Cook, S. B., Cantu, R. C., Ferrara, M. S., Guskiewicz, K. M., \& Myers, J. L. (2015). Early Results of a Helmetless-Tackling Intervention to Decrease 
Head Impacts in Football Players. Journal of Athletic Training, 50(12), 1219-1222. https://doi.org/10.4085/1062-6050-51.1.06

Szumilas, M. (2010). Explaining Odds Ratios. Journal of the Canadian Academy of Child and Adolescent Psychiatry, 19(3), 227-229.

Takhounts, E. G., Craig, C., Matthew J., Moorhouse, K., McFadden, J., \& Hasija, V. (2013). Development of brain injury criteria (BrIC). Stapp Car Crash Journal, 57, 243-266.

Takhounts, E. G., Eppinger, R. H., Campbell, J. Q., \& Tannous, R. E. (2003). On the development of the SIMon finite element head model. Stapp Car Crash Journal, 47, 107.

Takhounts, E. G., Hasija, V., \& Eppinger, R. H. (2009). Analysis of 3D Rigid Body Motion Using The Nine Accelerometer Array and the Randomoly Distributed in-plane Accelerometer Systems. In Proceedings of International Technical Conference on the Enhanced Safety of Vehicle. Stuttgart, Germany.

Takhounts, E. G., Hasija, V., Ridella, S. A., Rowson, S., \& Duma, S. M. (2011). Kinematic rotational brain injury criterion (BRIC). In Proceedings of the 22nd Enhanced Safety of Vehicles Conference. Paper.

Takhounts, E. G., Ridella, S. A., Hasija, V., Tannous, R. E., Campbell, J. Q., Malone, D., ... Duma, S. (2008). Investigation of traumatic brain injuries using the next generation of simulated injury monitor (SIMon) finite element head model. Stapp Car Crash Journal, 52,1 .

Taylor, C. A., Bell, J. M., Breiding, M. J., \& Xu, L. (2017). Traumatic Brain Injury-Related Emergency Department Visits, Hospitalizations, and Deaths — United States, 2007 and 2013. MMWR. Surveillance Summaries, 66(9), 1-16. https://doi.org/10.15585/mmwr.ss6609a1

The Nuremberg Code. (1949). In Trials of war criminals before the Nuremberg military tribunals under control council law 10 (Vol. 2, pp. 181-182). Washington, DC.

Thomson, R., Lovsund, P., Norin, H., Jakobsson, L., Bostrom, O., \& Haland, Y. (2001). Brain injuries in real world accidents-a multidisciplinary investigation. In Proceedings of the IRCOBI Conference, Isle of Man.

Thunnissen, J., Wismans, J., Ewing, C. L., \& Thomas, D. J. (1995). Human volunteer head-neck response in frontal flexion: a new analysis. In Proceedings: Stapp Car Crash Conference (Vol. 39, pp. 439-460). Society of Automotive Engineers SAE.

Thurman, D. J., Sniezek, J. E., Johnson, D., Greenspane, A., \& Smith, S. M. (1995). Guidelines for surveillance of central nervous system injury. Departament of Health and Human Services.

Versace, J. A. (1971). A review of the severity index. SAE Technical Paper. 
Viano, D. C., Casson, I. R., Pellman, E. J., Zhang, L., King, A. I., \& Yang, K. H. (2005). Concussion in Professional Football: Brain Responses by Finite Element Analysis: Part 9. Neurosurgery, 57(5), 891-916. https://doi.org/10.1227/01.NEU.0000186950.54075.3B

Viano, D. C., King, A. I., Melvin, J. W., \& Weber, K. (1989). Injury biomechanics research: An essential element in the prevention of trauma. Journal of Biomechanics, 22(5), 403-417. https://doi.org/10.1016/0021-9290(89)90201-7

Viano, D. C., Parenteau, C. S., Xu, L., \& Faul, M. (2017). Head injuries (TBI) to adults and children in motor vehicle crashes. Traffic Injury Prevention, 18(6), 616-622. https://doi.org/10.1080/15389588.2017.1285023

Vijayakumar, V., Scher, I., Gloeckner, D. C., Pierce, J., Bove, R., Young, D., \& Cargill, R. (2006). Head Kinematics and Upper Neck Loading During Simulated Low-Speed RearEnd Collisions: A Comparison With Vigorous Activities of Daily Living. https://doi.org/10.4271/2006-01-0247

Weis Jr, E. B., Clarke, N. P., \& Brinkley, J. W. (1963). Human response to several impact acceleration orientations and patterns. AIR FORCE AEROSPACE MEDICAL RESEARCH LAB WRIGHT-PATTERSON AFB OH.

Wilcox, B. J., Beckwith, J. G., Greenwald, R. M., Chu, J. J., McAllister, T. W., Flashman, L. A., ... Crisco, J. J. (2014a). Head impact exposure in male and female collegiate ice hockey players. Journal of Biomechanics, 47(1), 109-114. https://doi.org/10.1016/j.jbiomech.2013.10.004

Wilcox, B. J., Machan, J. T., Beckwith, J. G., Greenwald, R. M., Burmeister, E., \& Crisco, J. J. (2014b). Head-Impact Mechanisms in Men's and Women's Collegiate Ice Hockey. Journal of Athletic Training, 49(4), 514-520. https://doi.org/10.4085/1062-6050-49.3.19

Willinger, R., \& Baumgartner, D. (2003). Human head tolerance limits to specific injury mechanisms. International Journal of Crashworthiness, 8(6), 605-617.

Willinger, R., Baumgartner, D., Chinn, B., \& Neale, M. (2000). Head tolerance limits derived from numerical replication of real world accidents. In Proceedings of the International Research Council on the Biomechanics of Injury conference (Vol. 28, pp. 209-221). International Research Council on Biomechanics of Injury.

Wismans, J., Philippens, M., Van Oorschot, E., Kallieris, D., \& Mattern, R. (1987). Comparison of human volunteer and cadaver head-neck response in frontal flexion. SAE Technical Paper.

Wismans, J., \& Spenny, C. H. (1984). Head-neck response in frontal flexion. SAE Technical Paper.

Wong, R. H., Wong, A. K., \& Bailes, J. E. (2014). Frequency, magnitude, and distribution of head impacts in Pop Warner football: The cumulative burden. Clinical Neurology and Neurosurgery, 118, 1-4. https://doi.org/10.1016/j.clineuro.2013.11.036 
Wu, L. C., Nangia, V., Bui, K., Hammoor, B., Kurt, M., Hernandez, F., ... Camarillo, D. B. (2016). In Vivo Evaluation of Wearable Head Impact Sensors. Annals of Biomedical Engineering, 44(4), 1234-1245. https://doi.org/10.1007/s10439-015-1423-3

Wu, L. C., Zarnescu, L., Nangia, V., Cam, B., \& Camarillo, D. B. (2014). A Head Impact Detection System Using SVM Classification and Proximity Sensing in an Instrumented Mouthguard. IEEE Transactions on Biomedical Engineering, 61(11), 2659-2668. https://doi.org/10.1109/TBME.2014.2320153

Young, T. J., Daniel, R. W., Rowson, S., \& Duma, S. M. (2014). Head Impact Exposure in Youth Football: Elementary School Ages 7-8 Years and the Effect of Returning Players. Clinical Journal of Sport Medicine, 24(5), 416-421. https://doi.org/10.1097/JSM.0000000000000055

Zaborowski, A. (1964). Human Tolerance to Lateral Impact With Lap Belt Only< xref rid=. SAE Technical Paper.

Zaborowski, A. (1965). Lateral Impact Studies-Lap Belt Shoulder Harness Investigations. SAE Technical Paper.

Zhang, L., Yang, K. H., \& King, A. I. (2004). A proposed injury threshold for mild traumatic brain injury. Journal of Biomechanical Engineering, 126(2), 226-236.

Zhang, F., \& Chen, C.-L. (2013). NASS-CDS: sample design and weights.

Zhao, W., \& Ji, S. (2016). Real-time, whole-brain, temporally resolved pressure responses in translational head impact. Interface Focus, 6(1), 20150091.

Zhao, W., Kuo, C., Wu, L., Camarillo, D. B., \& Ji, S. (2017). Performance Evaluation of a Precomputed Brain Response Atlas in Dummy Head Impacts. Annals of Biomedical Engineering. https://doi.org/10.1007/s10439-017-1888-3 

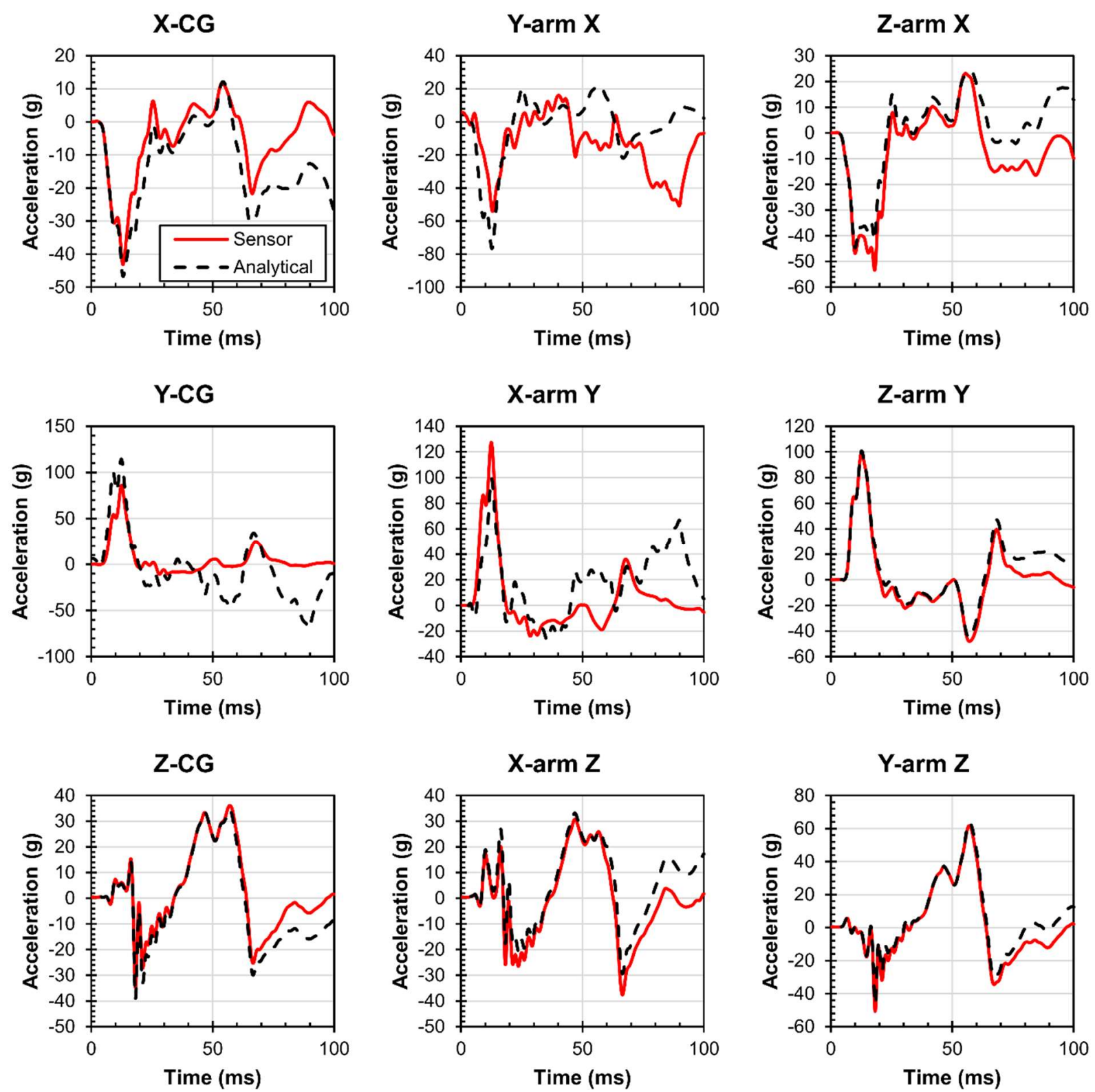

Figure A1. Example of an inconsistent NAP array. The analytical traces were calculated for each sensor as a function of the other eight sensor measures using an algorithm developed by Takhounts et al. (2009). Clearly, in this case, the analytical solutions do not align with the measured traces, indicating an error from one or more accelerometer measures. 

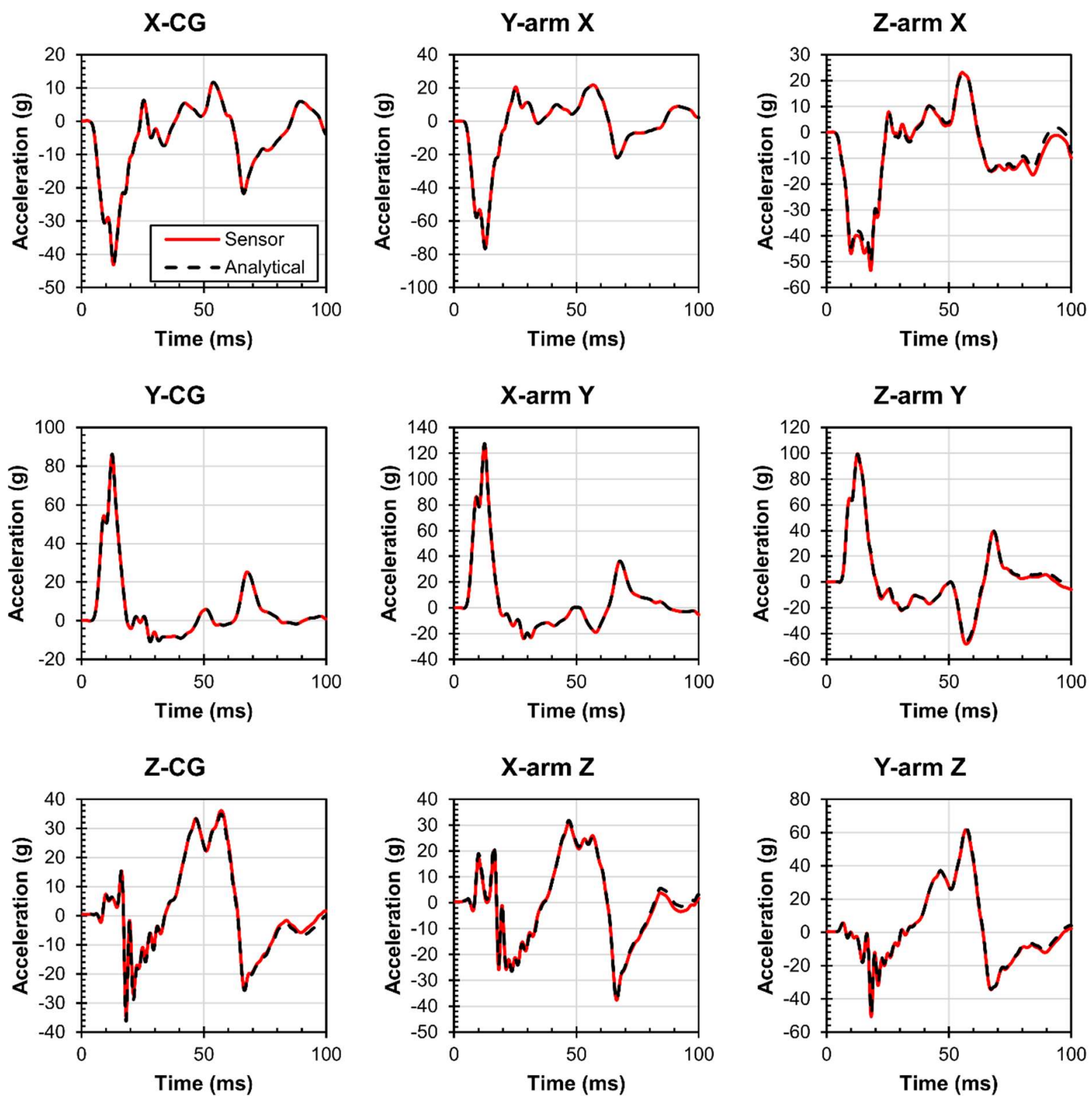

Figure A2. Example of a corrected (and consistent) NAP array. The analytical solutions align with the sensor measures. In this case, the Y-arm X channel was identified as inconsistent sensor from Figure A1; therefore its measured trace was replaced with the analytical solution from the previous figure and analytical traces were recalculated. 

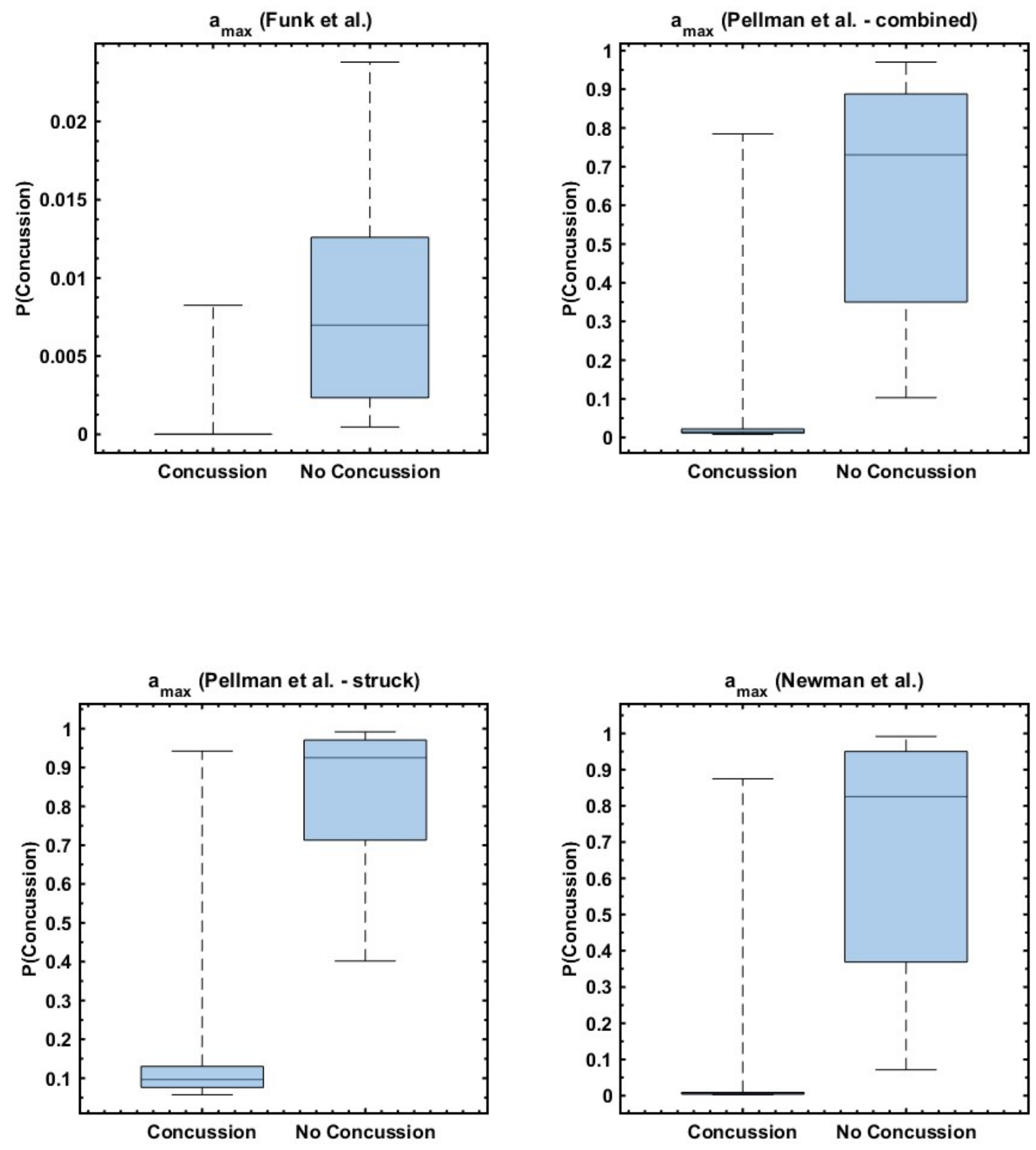

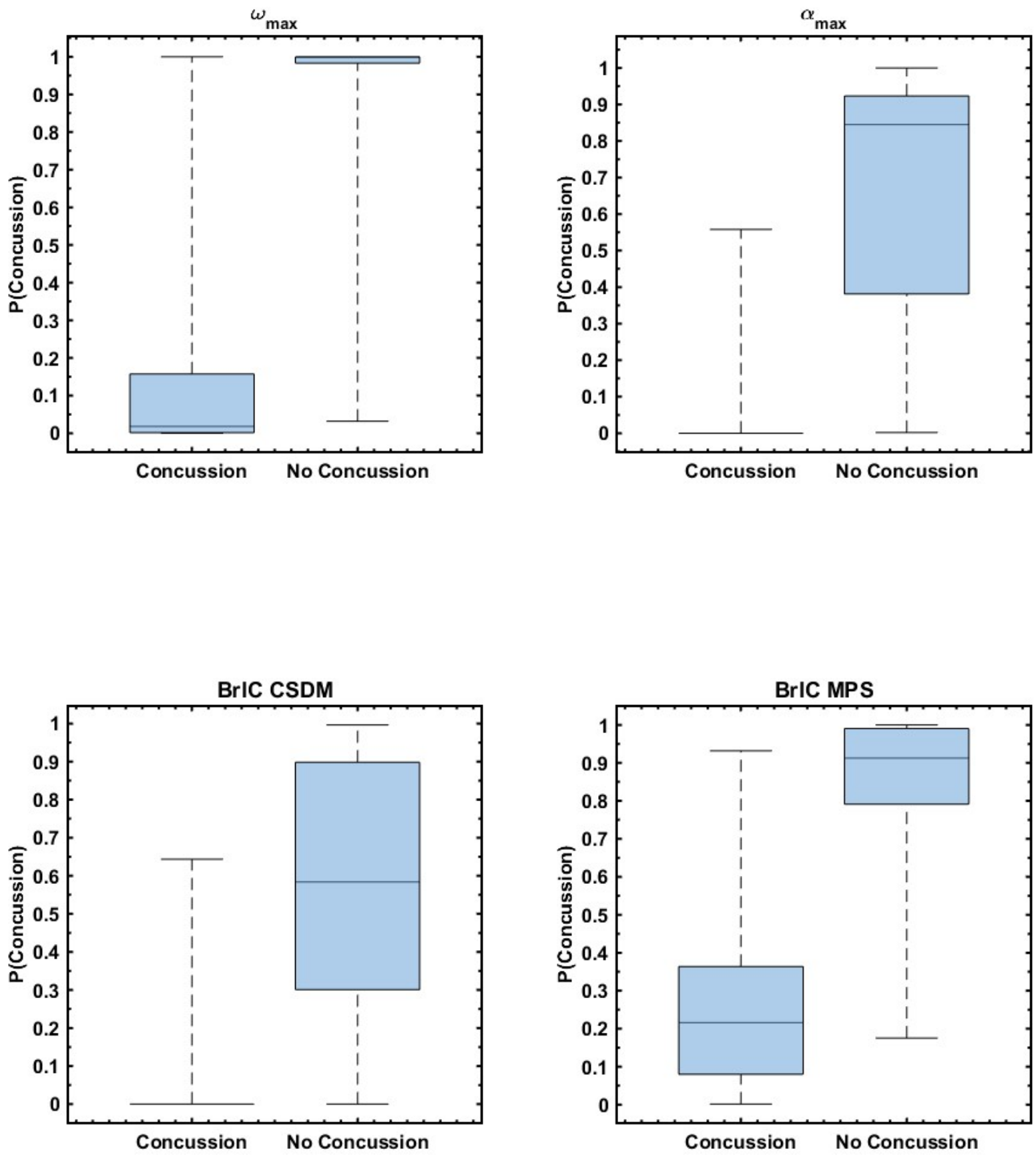

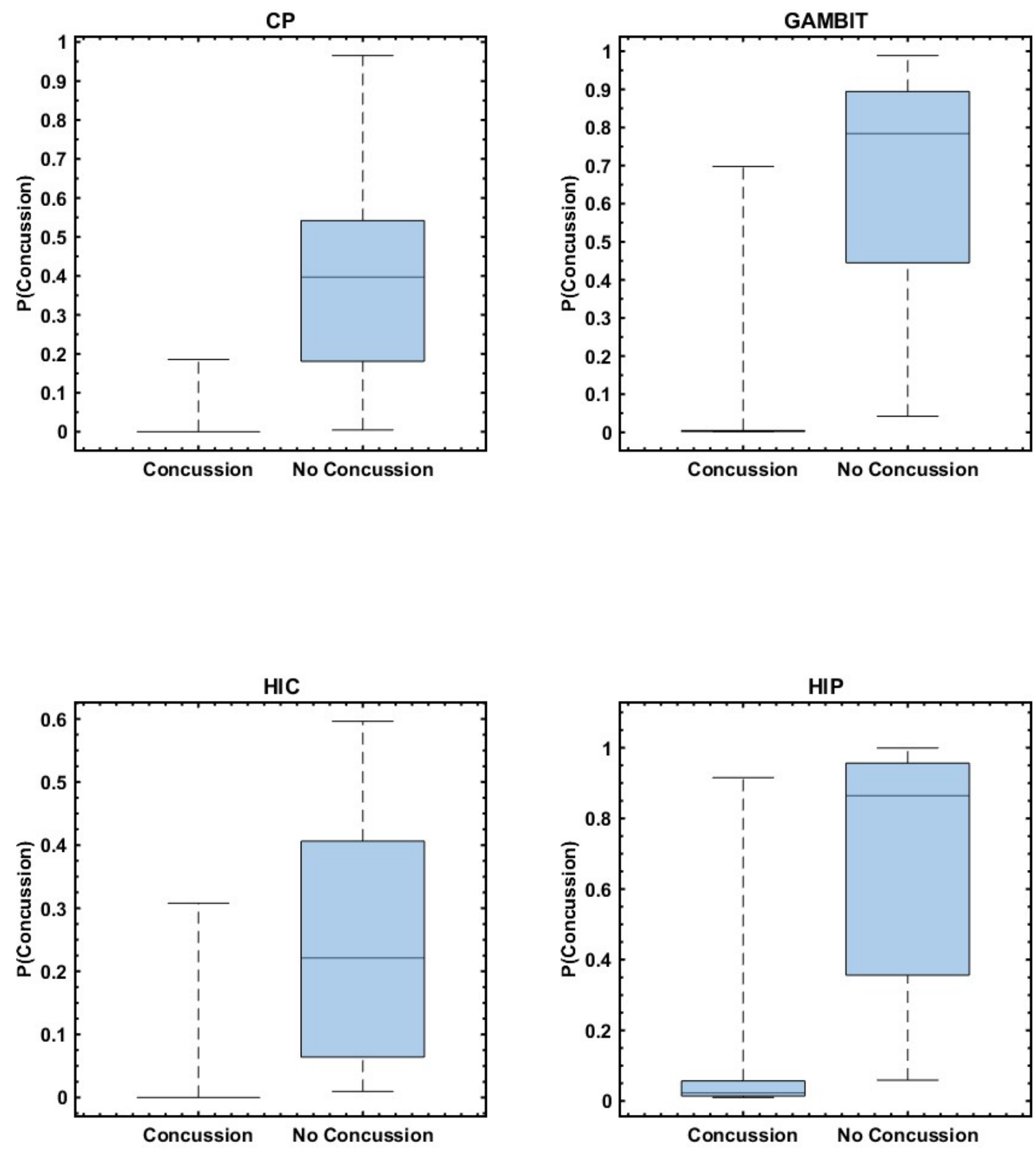

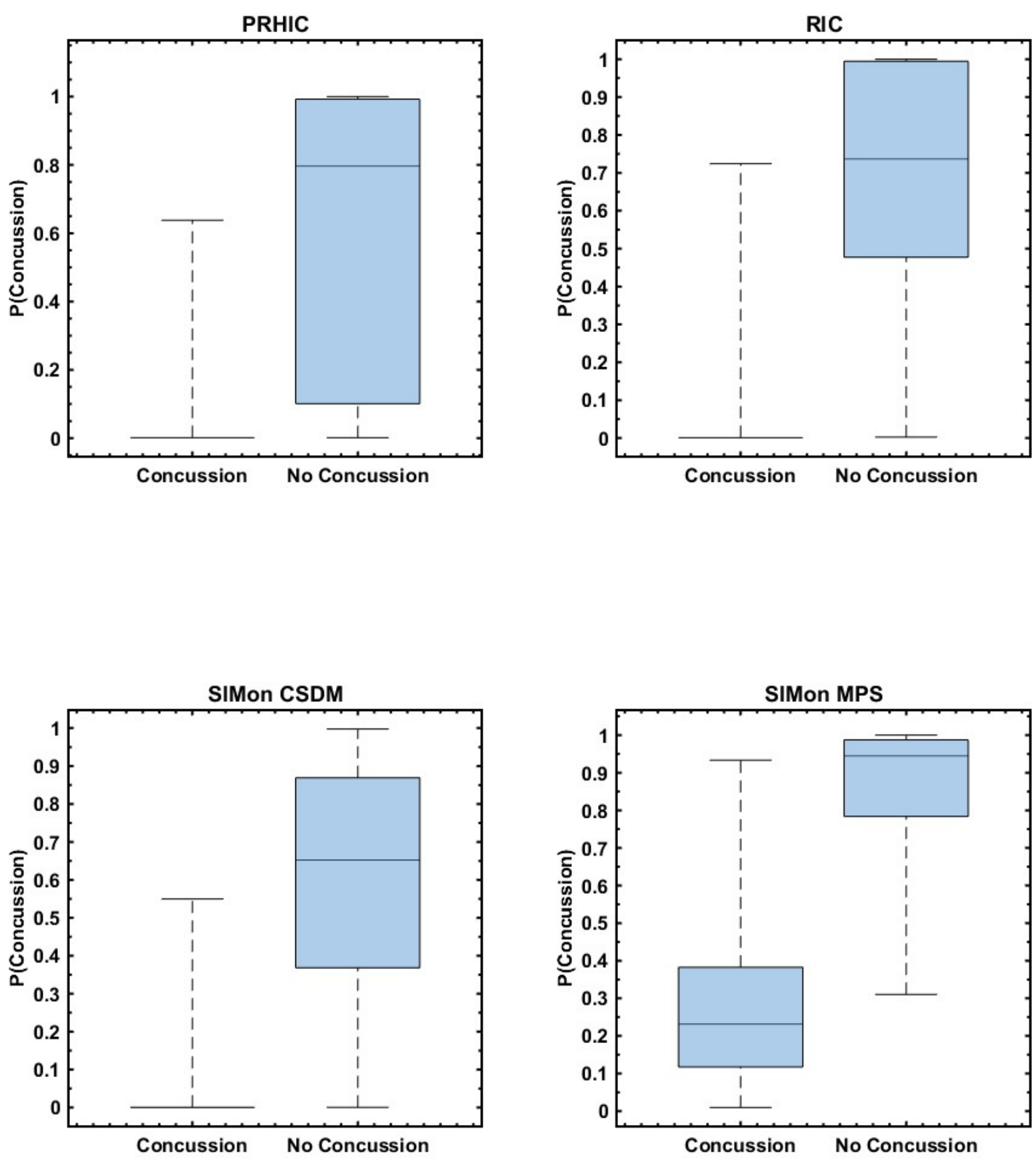